\title{
Out With The Old, In With The New:
}

\section{Clinicoserologic Classification of Inflammatory Myositis And Their \\ Imaging Findings}

Dr. Davis Holmes

Dr. Isabelle Dupuis

Dr. Kun Huang

Dr. Natasha Dehghan

Dr. Darra Murphy 
The authors do not have a relationship with a for-profit and/or a not-for-profit organization to disclose. 


\section{Goals \& Objectives}

1. Understand the connection between the Bohan and Peter classification of myositis and the evolving clinicoserologic correlation of autoimmune inflammatory myositis

2. Review of inflammatory myositis based on clinicoserologic phenotypes

3. Describe the major organ systems involved and the imaging manifestations of the major clinicoserologic subtypes

\section{Target Audience}

Residents, Fellows and Staff Radiologists 


\section{Background}

\section{Bohan and Peter Classification (1975)}

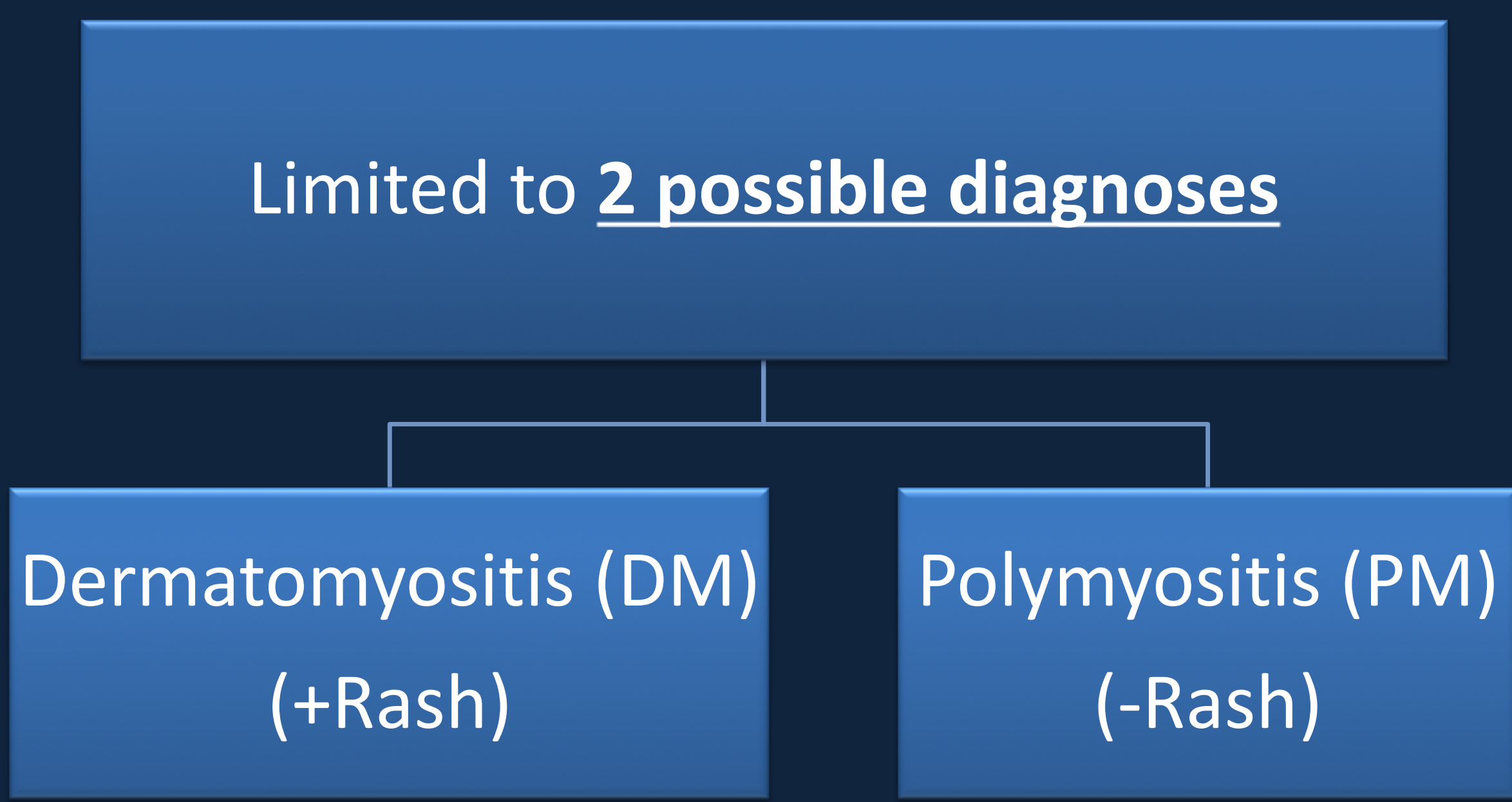

Key components:

- Proximal symmetrical muscle weakness

- Biochemical findings

- EMG

- Biopsy

- Cutaneous changes

- Heliotrope Rash

- Gottron's sign 


\section{What have we learned since $1975 ?$}

- DM rash is a poor clinical feature to segment the IIMs

- DM rash is present in many patients with additional clinical features such as interstitial lung disease (ILD), arthritis, calcinosis

- Patients previously diagnosed with "polymyositis" likely misdiagnosed

- Inclusion body myositis and immune-mediated necrotizing myositis both often meet the criteria for "Polymyositis"

- Specific biopsy features of the idiopathic inflammatory myopathies (IIM) cannot be agreed upon

- Numerous autoantibodies have strongly identified correlations with IIMs

Conclusion:

Serology combined with extra-muscular clinical features provide the most robust method of identifying patient groups with a similar clinical course 


\section{What is a 'dermatomyositis rash'?}

Key components:

- Heliotrope

- Shawl sign

- V-sign

- Gottron's papules

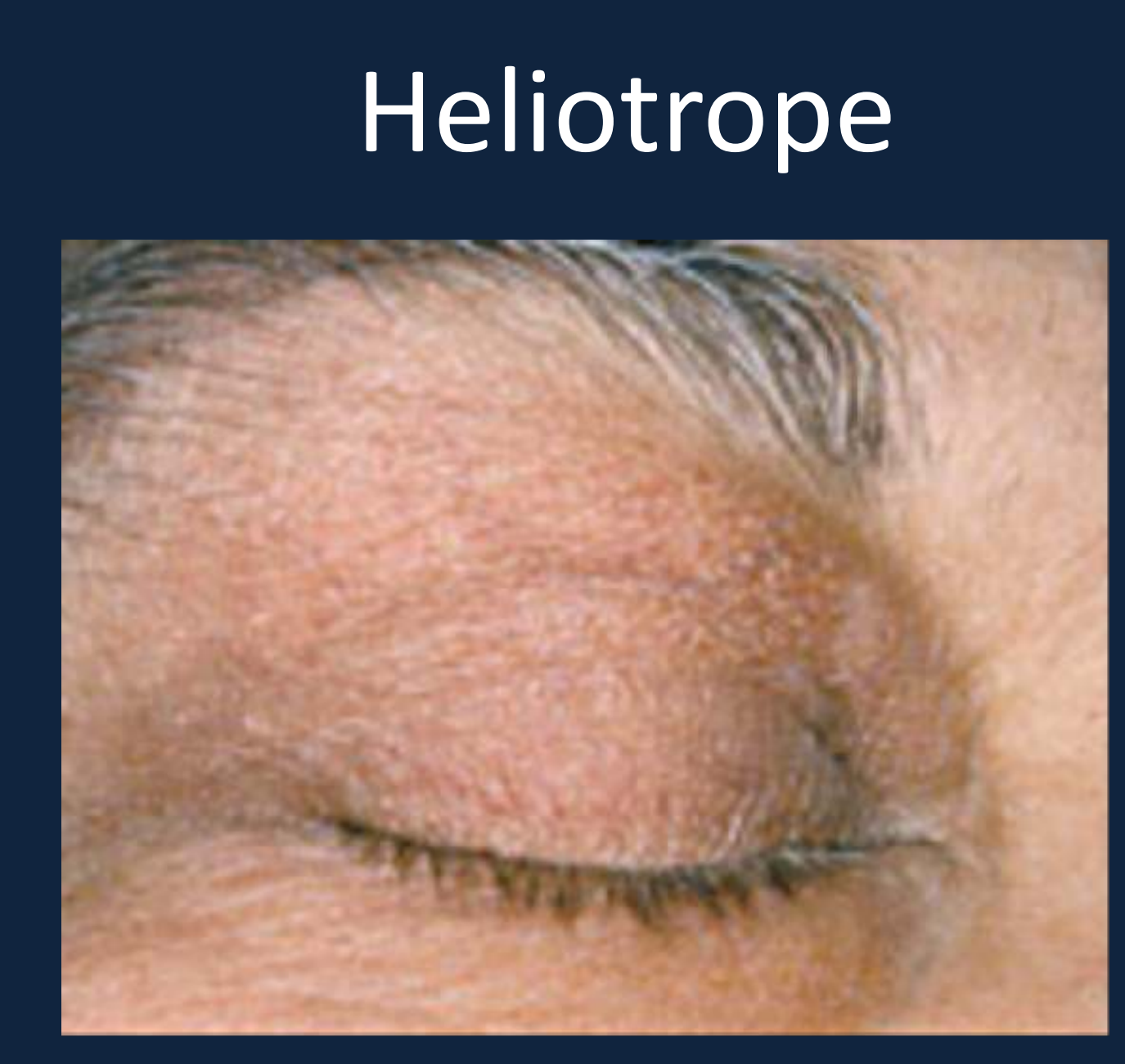

Adapated from Koler et al.

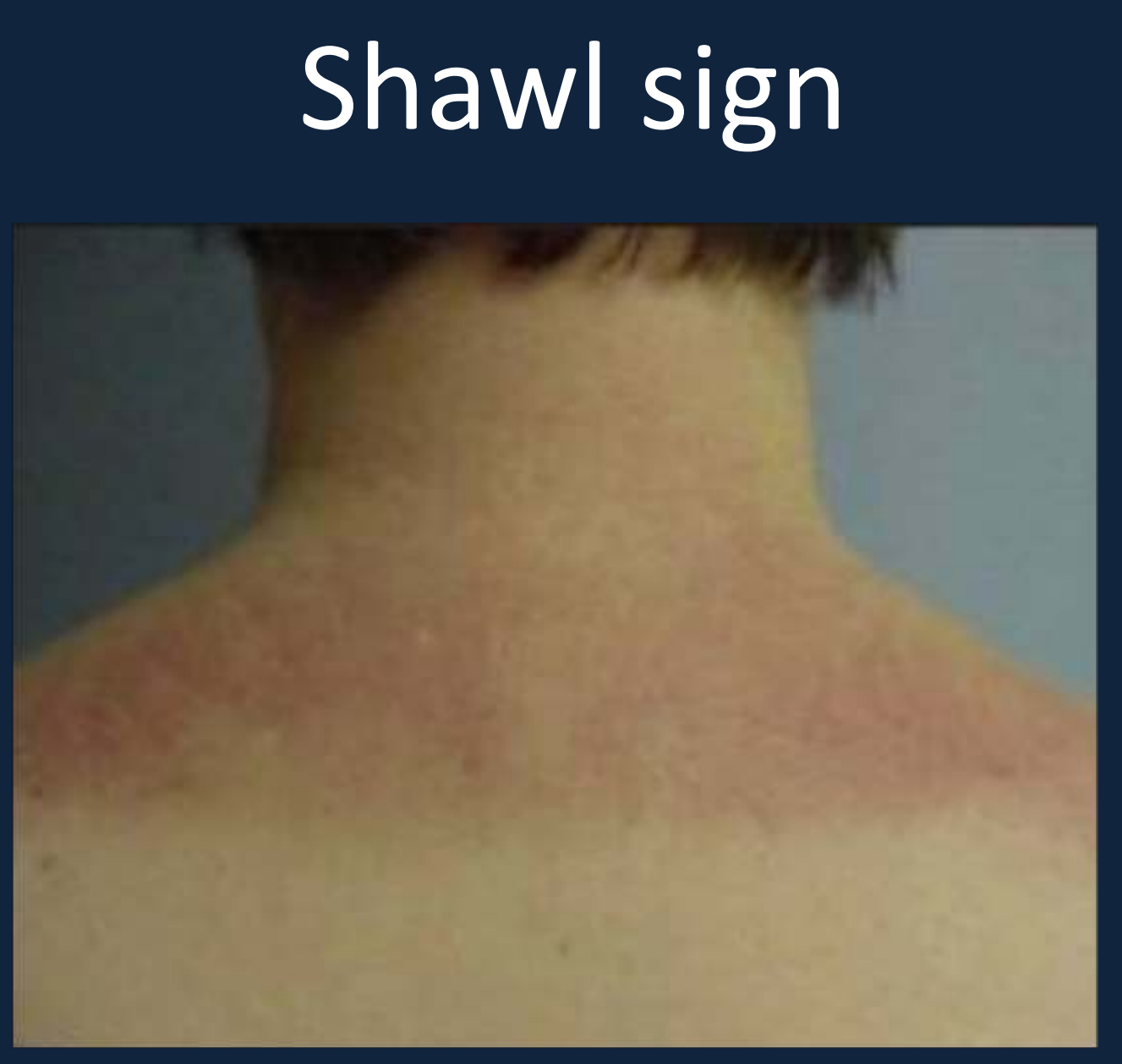

Adapted from Marvi et al.

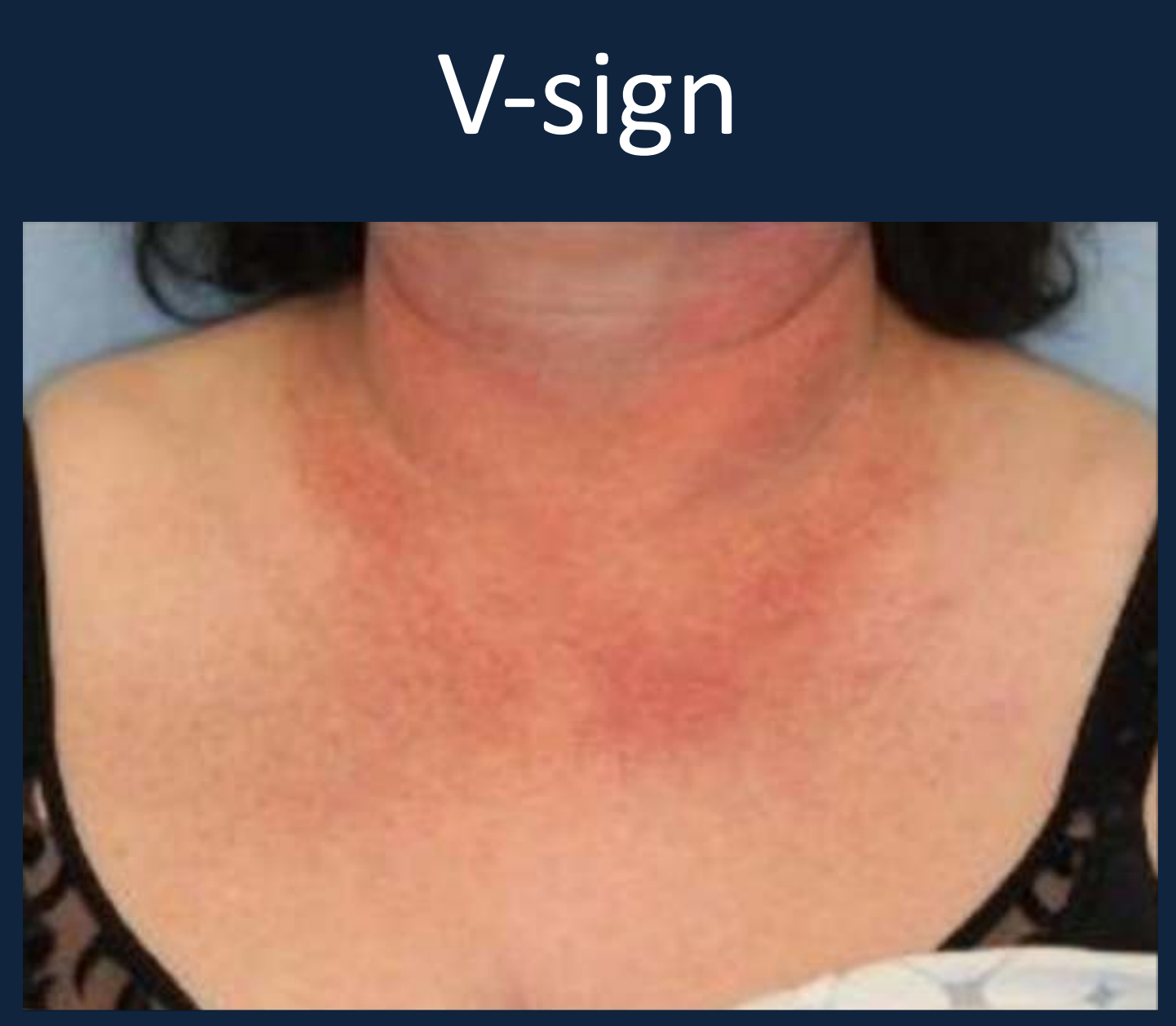

Adapted from Marvi et al.
Gottron's papules

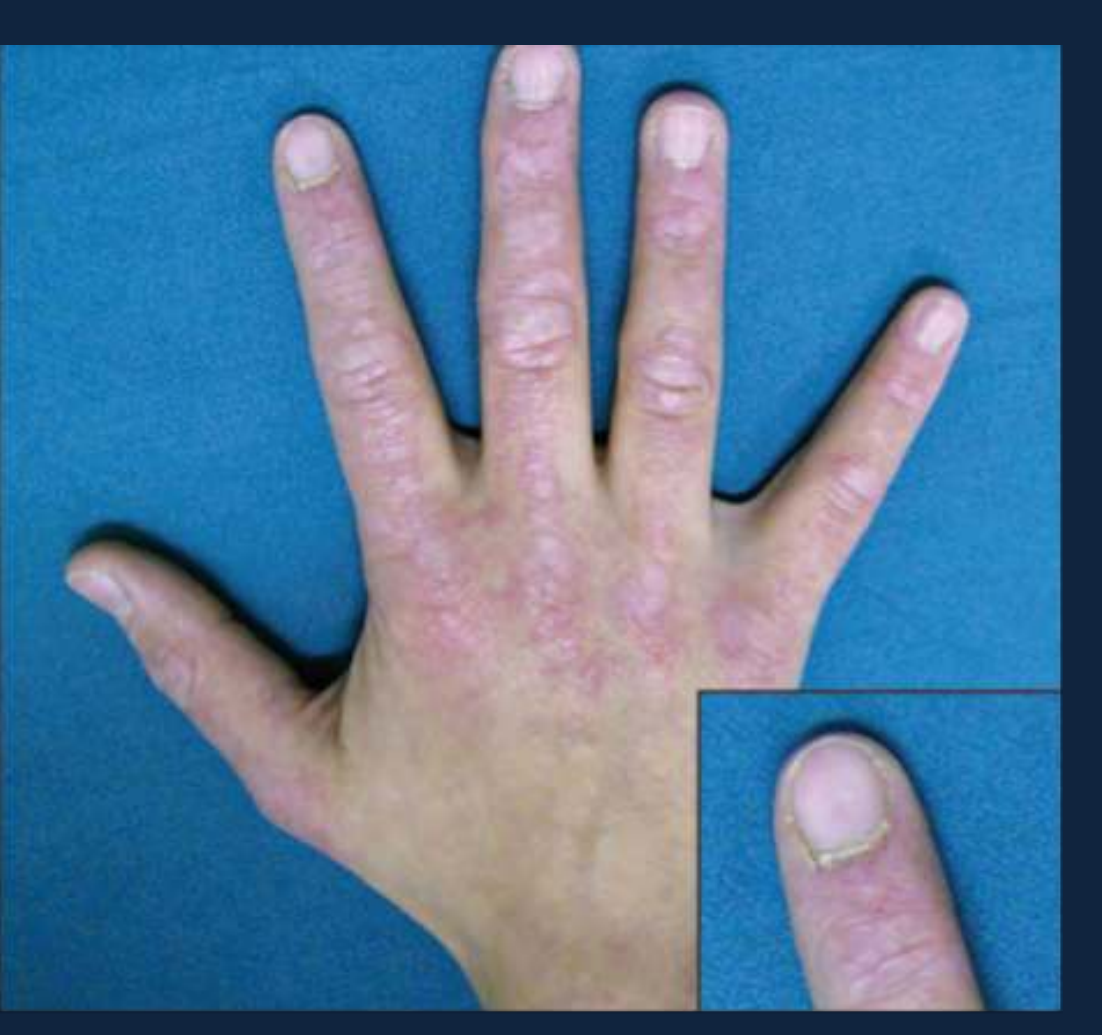

Adapted from Ricceri et al. 


\section{New Paradigm: Clinicoserologic Phenotypes}

- Inflammatory myositis patients demonstrate features of other rheumatologic conditions

- Patients diagnosed with polymyositis (PM) develop signs/symptoms not captured by Bohan and Peter Classification

- Auto-antibodies have been discovered that correlate with extramuscular manifestations 


\section{Dermatomyositis \& Overlap Myositis}

Patients with suspected dermatomyositis (DM) that develop clinical features seen in other connective tissue diseases (CTD) are categorized as overlap myositis (OM)

\section{Dermatomyositis (DM)}

- Rash: Non-transient \& develops before myositis

- Serology specific for DM

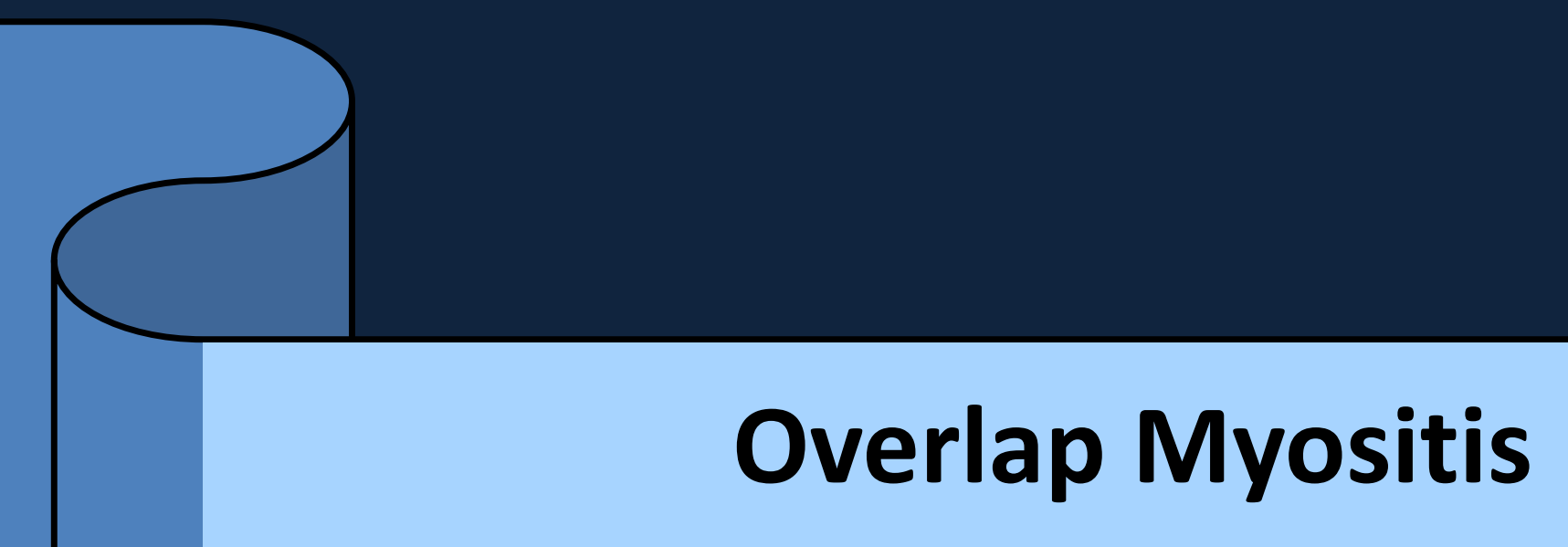

- Rash: Transient \& develops after myositis

- Features of other CTD (e.g. Raynaud's, arthritis, digital ulcers)

- Serology specific for OM subtypes 


\section{Primer on Autoantibodies for the Radiologist}

Myositis-specific antibodies (MSAs)

- Do not co-exist with other CTD antibodies or other MSAs in the same patient

Examples

- Strong associations with particular IIM subtypes

- Predictable pattern of disease features and risk of complications

Myositis-associated antibodies (MAAs)

- Can co-exist with other CTD antibodies and other MSAs

- Moderate associations with particular clinical features

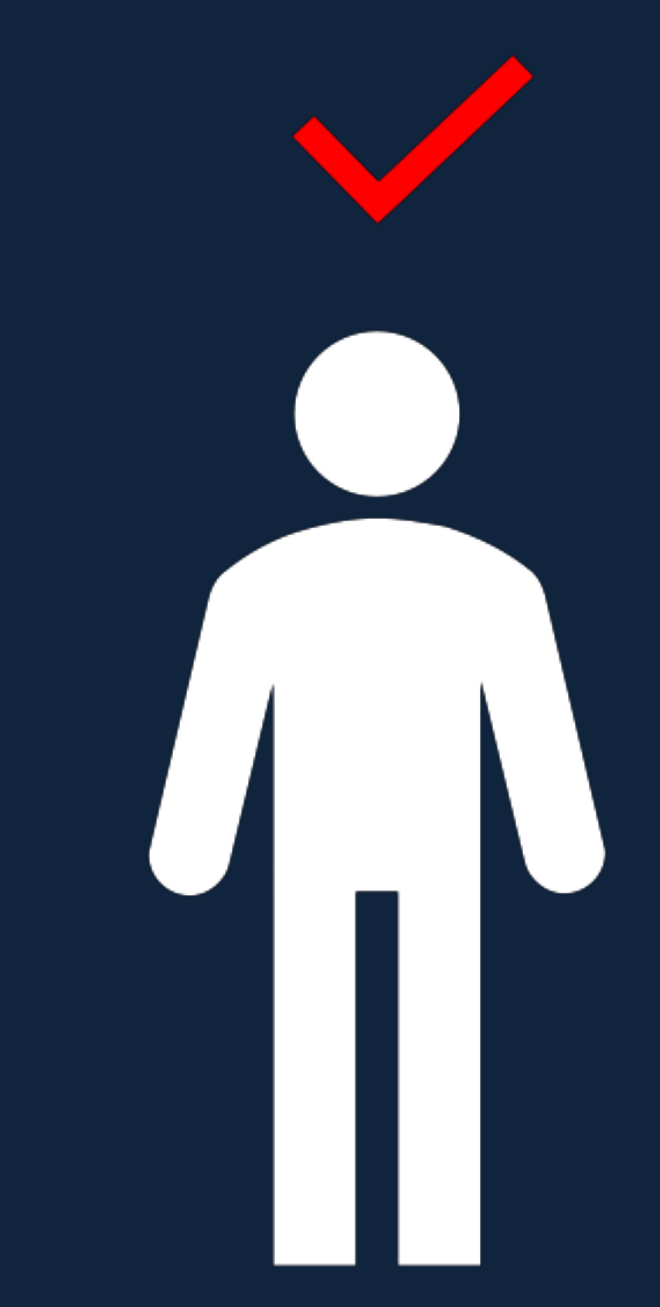

anti-Jo-1

Okay, because no 'conflicting Abs. 2 MSAs will not be positive in same pt.

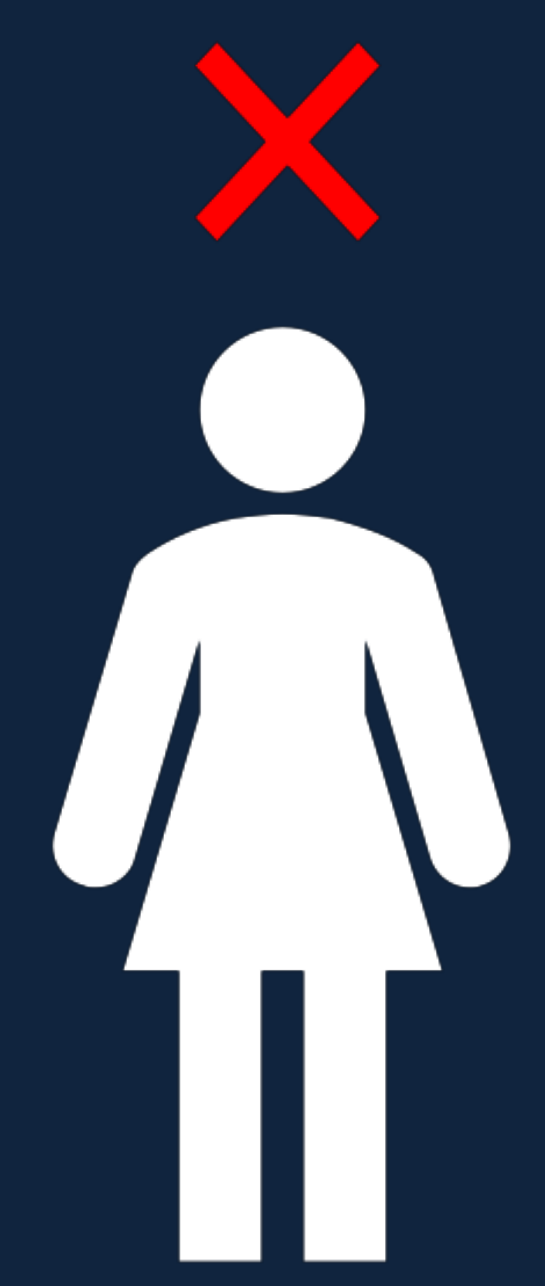

anti-Jo-1 anti-TIF1g
MAAs

anti-PM/Scl

anti-Ku

\begin{tabular}{l|l|}
\hline MSAs & MAAs \\
\hline anti-Jo-1 & anti-PM/Scl \\
anti-TIF1g & anti-Ku
\end{tabular}

Not seen in same Okay, because antipatient because dsDNA is Ab for SLE and anti-Ku is an MAA (not an MSA). 


\section{Clinicoserologic Phenotypes}

Overlap Myositis (OM)

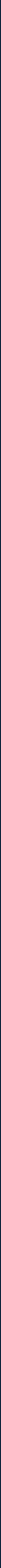




\section{What is Classic Dermatomyositis (DM)?}

Clinical Features

- Prominent DM rash at presentation

- DM rash is relatively resilient to treatment

- Myalgias/myositis

- No ILD

- No arthritis
3 Myositis-Specific Autoantibodies

\begin{tabular}{|l|l|}
\hline Antibody & \multicolumn{1}{c}{ Associations } \\
Anti-Mi2 & Classic DM - skin and proximal muscle weakness \\
& More resistant to corticosteroids \\
\hline Anti-NXP2 & $\begin{array}{l}\text { Classic DM with additional distal muscle weakness, } \\
\text { greater subcutaneous edema, calcinosis and } \\
\text { dysphagia }\end{array}$ \\
Anti-TIF1g & $\begin{array}{l}\text { Possible increased risk of malignancy within } 3 \text { years } \\
\text { Increased risk of cancer within } 3 \text { years }\end{array}$
\end{tabular}

-myositis-specific Ab are present in up to 70\% of patients with classic DM, which helps differentiate classic DM from overlap myositis. 


\section{What is Clinically Amyopathic DM (CADM)?}

Synonym = Amyopathic or Hypomyopathic Dermatomyositis

Myositis-specific Antibodies: anti-MDA5 (frequently present) and anti-SAE

- Lower rate of 'weakness'

- Higher rates of polyarthritis (82\%)

- Higher rates of digital ulcers

\section{Clinical Features}

- DM rash and digital ulcers

- Minimal muscle involvement

- ILD present (rapidly progressing ILD)

- No arthropathy 


\section{What is Anti-Synthetase Syndrome?}

\section{Myositis-specific Antibodies (8 total)}

- anti-Jo1

- anti-PL7

- anti-PL12

- 5 less well-studied Abs (low prevalence)

Clinical Features: "FIRMA" *

- Fever

- ILD

- Raynaud's

- Mechanic's hands

- Arthritis : RA pattern, subluxing or swelling

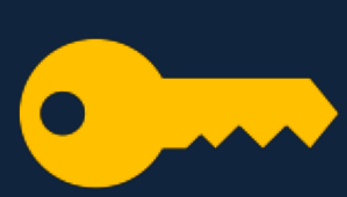

Proportion of each clinical feature in 'FIRMA is dictated by the specific serology. E.g., antiPL7 ASS can present with minimal myositis
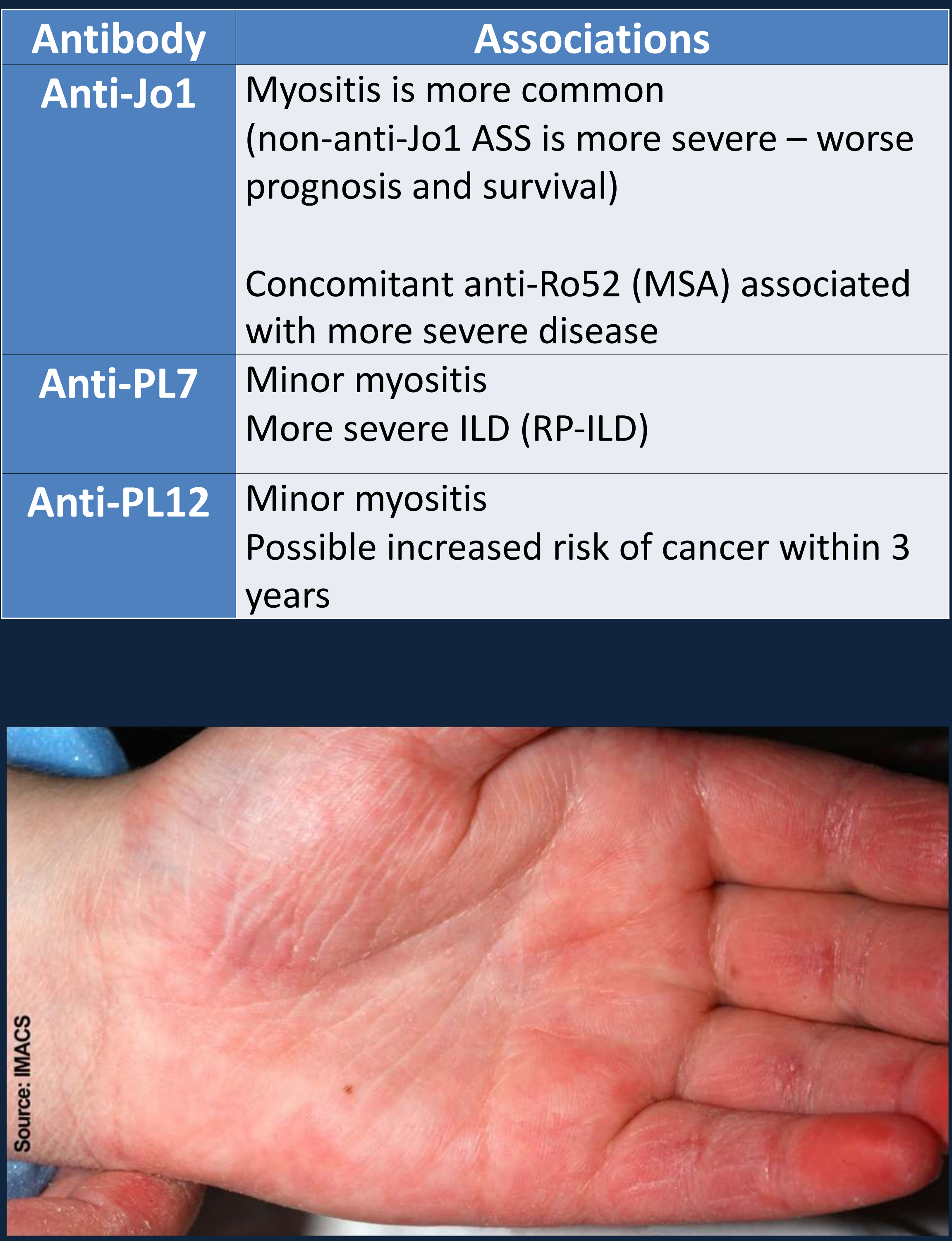

Mechanic's hands 


\section{What is Overlap Myositis (OM) - Connective Tissue Diseases (CTD)?}

\section{Subtypes:}

- OM-Scleroderma (OM-SSc)- most common 1/3

- OM-Systemic Lupus Erythematosus (OM-SLE)

- OM-Rheumatoid Arthritis (OM-RA)

- OM-Sjogren's syndrome (OM-SS)

- OM-Mixed Connective Tissue Disease (OMMCTD)

Other types of 'OM' best described by serology when overlap with another CTD is not present

OM can share many features with other CTD while not meeting full criteria for those diseases

$\therefore$ Overlap myositis can be both a 'category' of autoimmune myositis or a specific diagnosis if specific CTD criteria are met

\section{OM-Scleroderma (OM-SSC)}

- Also termed scleromyositis

- Many features of anti-synthetase syndrome:

- ILD (60\%)

- Arthralgia/arthritis (35\%)

- Raynaud's phenomenon (40\%)

- Mechanic's hands (10\%)

\section{Antibody}

Associations

Anti-PM/Scl Develop many of the sclerodermatous complications

Anti-Ku When criteria for SSc are met, most often means it will be complicated by myositis

SSc patients with anti-Ku often are not positive for other classic serologic markers (anti-centromere and anti-topoisomerase) 


\section{What is Immune-Mediated Necrotizing Myopathy (IMNM)?}

Myositis-specific Antibodies

- Anti-SRP (higher CK)

- Anti-HMGCR

\section{Presentations}

(1) Subacute (70-80\%): Max severity within months

(2) Slow progressive (20-30\%): Mimics muscular dystrophy

\section{Clinical features:}

- Severe myositis (very high CK)

- Axial weakness, especially with anti-SRP

- Head drop syndrome in $7 \%$

- More refractory to treatment

- Classic 'overlap' features (ILD, arthralgia, rash) very rare

\section{Statin-Induced?}

- Many anti-HMGCR IMNM associated with statin exposure!

- Unexposed cases may be due to natural statins in diet

Pathology:

Very different from DM/OM with paucity of inflammatory cells and marked necrosis/regenerating myocytes 


\section{What is Inclusion Body Myositis (IBM)?}

Myositis-specific Antibody

- Anti-c5N1a

Clinical Features

- Muscular findings only (esp. distal thigh and anterior forearm)

- No extra-muscular manifestations!

- ILD: none!

- Bone: none!

- Skin: none! 
Imaging in Idiopathic Inflammatory Myopathies (IIM)

MSK Imaging

- Muscular/fascial involvement

- Arthropathies

- Soft tissue calcinosis 


\section{MRI Methods}

- Recommended Sequences

- Cor \& Ax T1 (Atrophy)

- Cor \& Ax T2FS or STIR (Edema)

- $\mathrm{Gd} \rightarrow$ No additional diagnostic information

- Imaged Regions

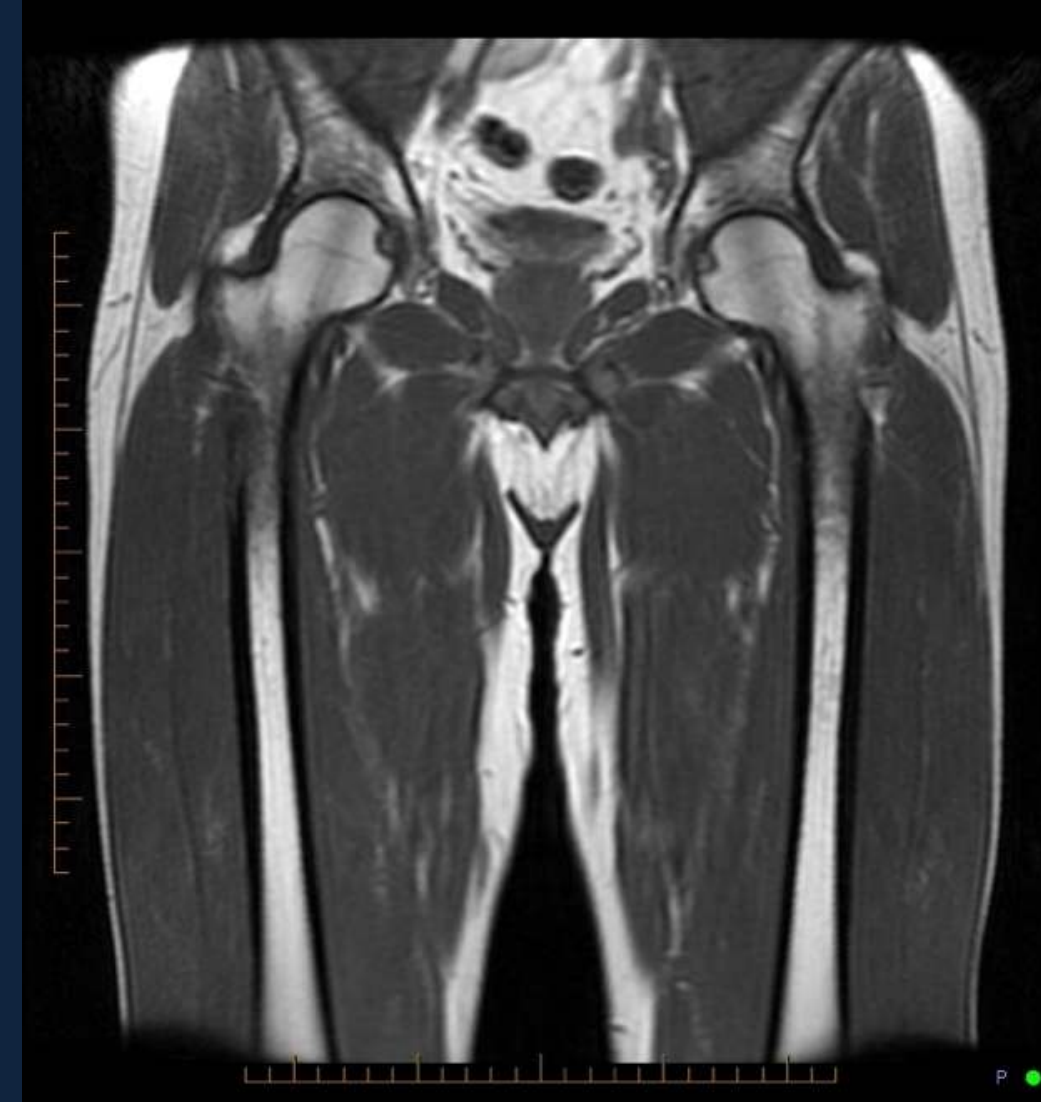

Cor T1

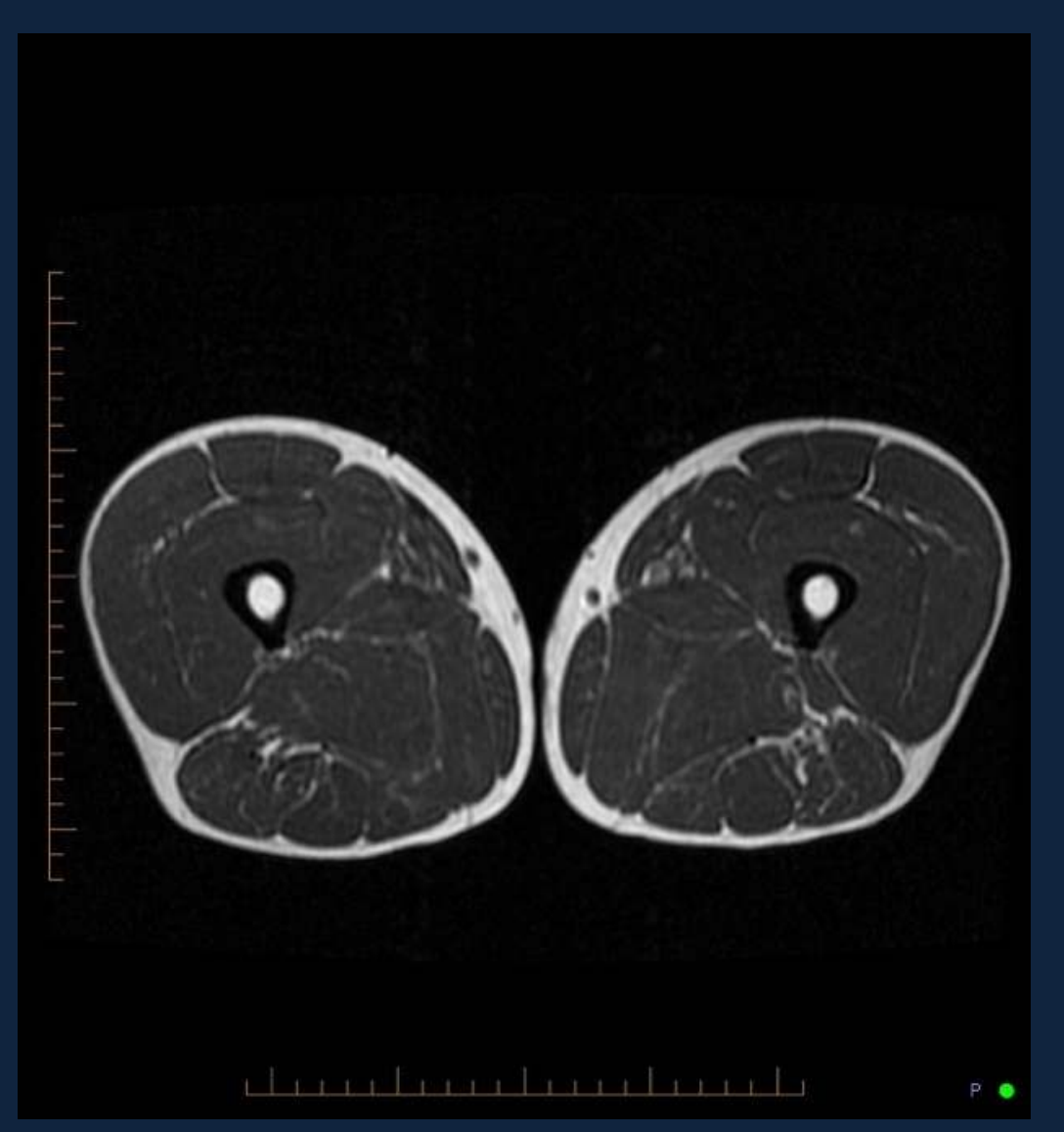

Ax T1

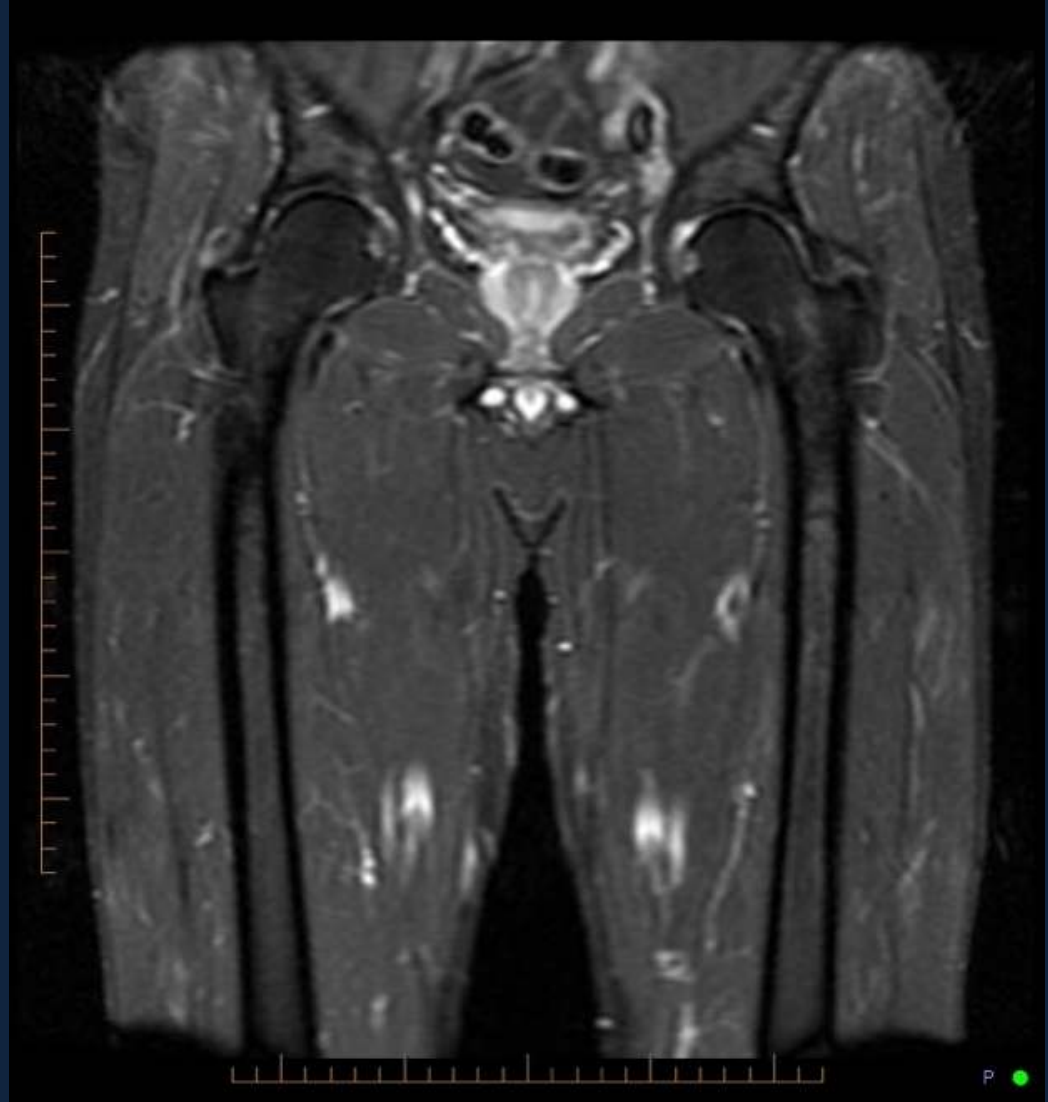

Cor STIR

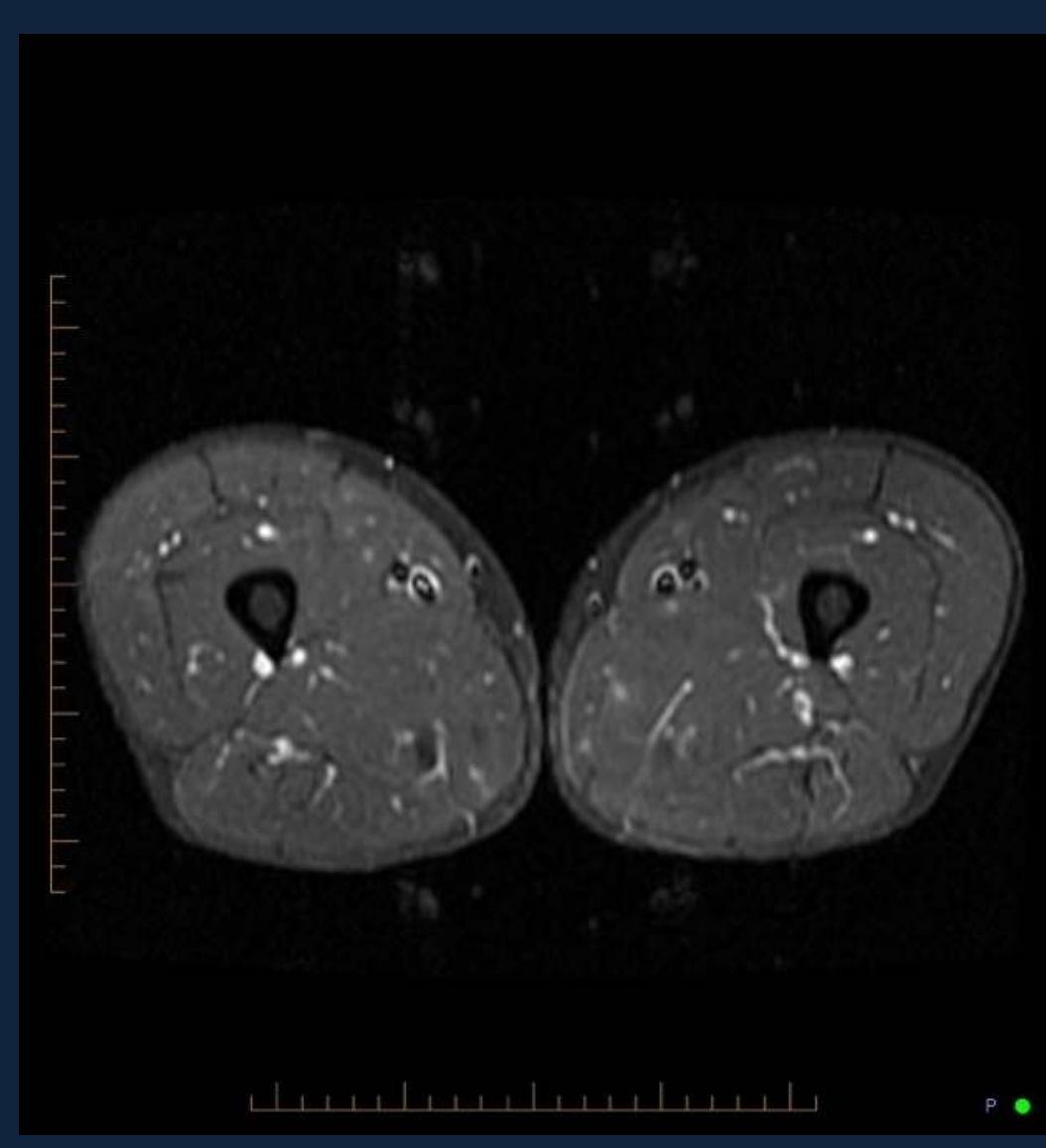

Ax STIR 


\section{Ultrasound Methods}

Fairly sensitive and specific to detect abnormal muscles

Features:

- Edema:

- muscle volume, mild hyperechogenicity, hyperememia (power doppler)

- Atrophy:

- $\downarrow$ muscle volume, marked echogenicity, low vascularity

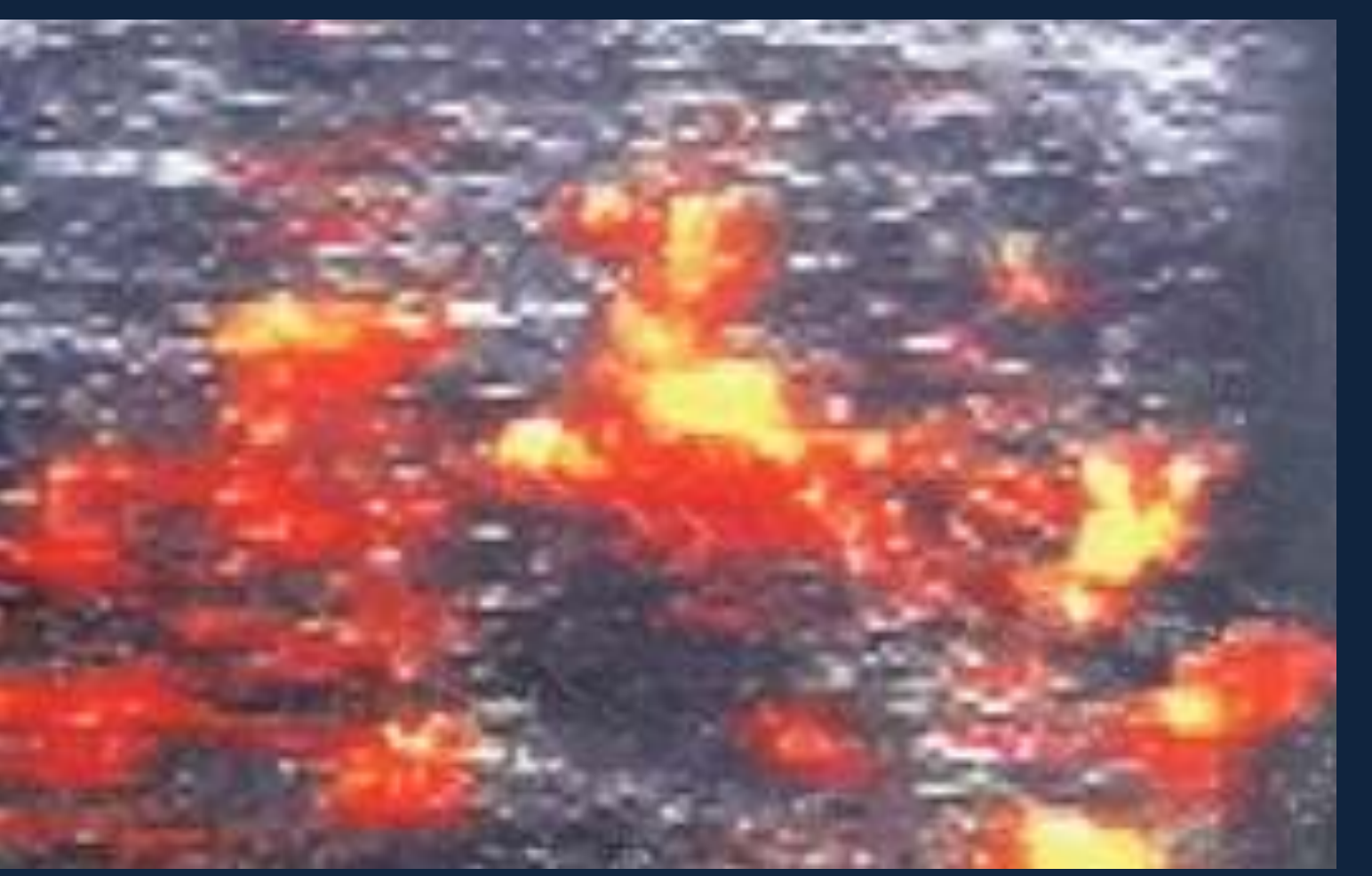

US upper arm:

Biceps in DM patient demonstrating increased vascularity

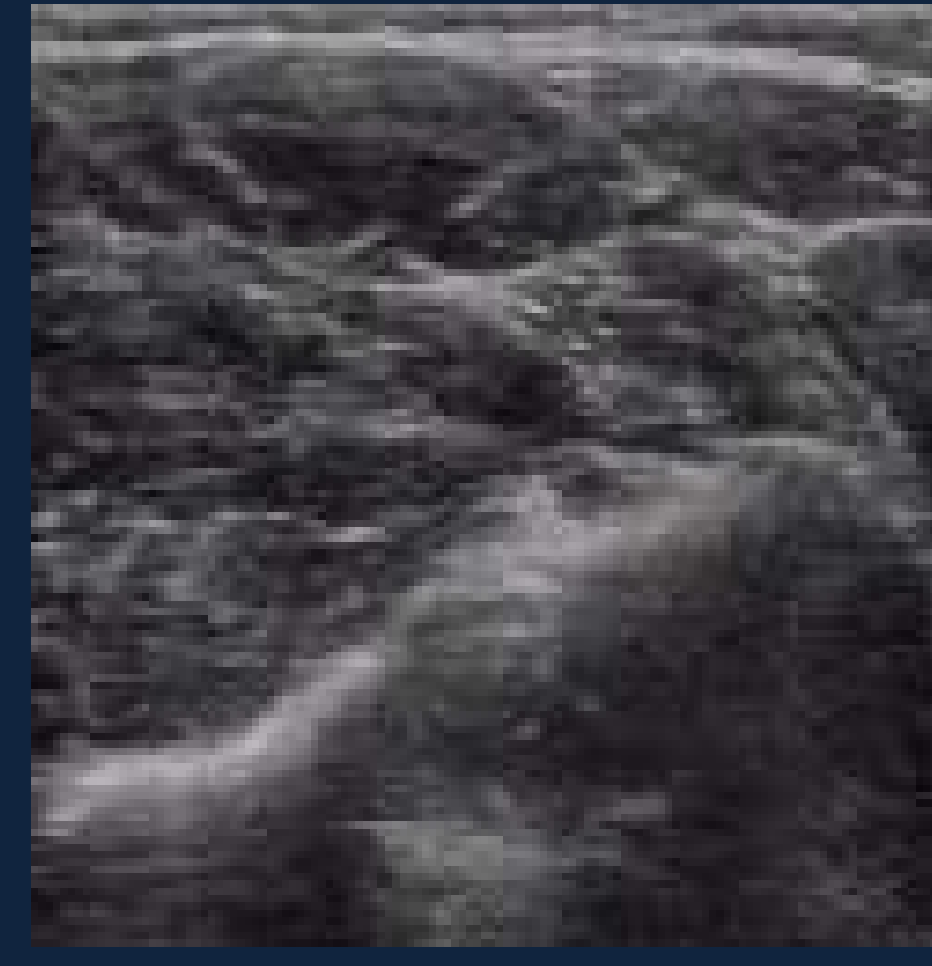

US Forearm: Normal FCR

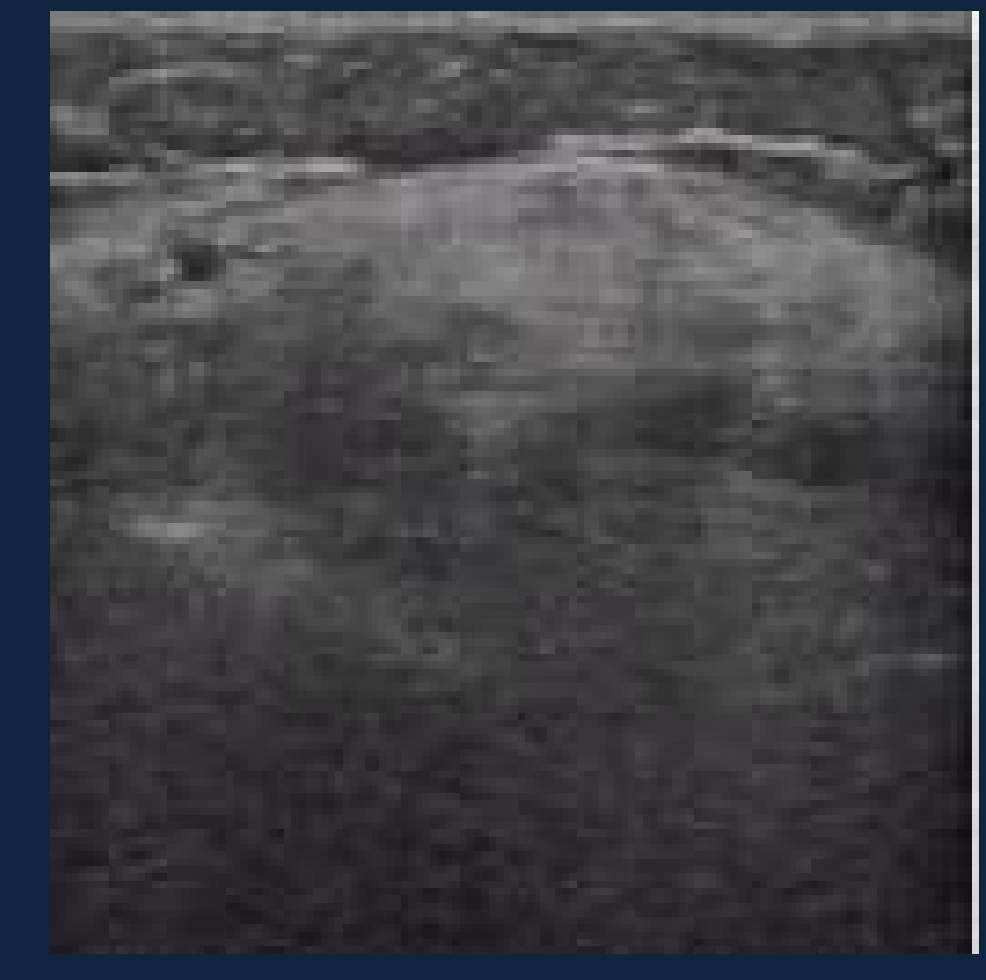

US Forearm:

FCR in patient with IBM

demonstrating atrophy and increased echogenicity 


\section{Nuclear Techniques}

FDG PET, Ga67 SPECT and Tc99m-PYP scintigraphy have been used to evaluate muscle activity

FDG PET/CT:

- Often performed for malignancy screening

- Less validated than MRI for myositis

- Benefit of imaging entire body (distribution of activity \& severity)
FDG PET/CT - PM patient with heterogeneous muscle activity without structural changes on $\mathrm{CT}$

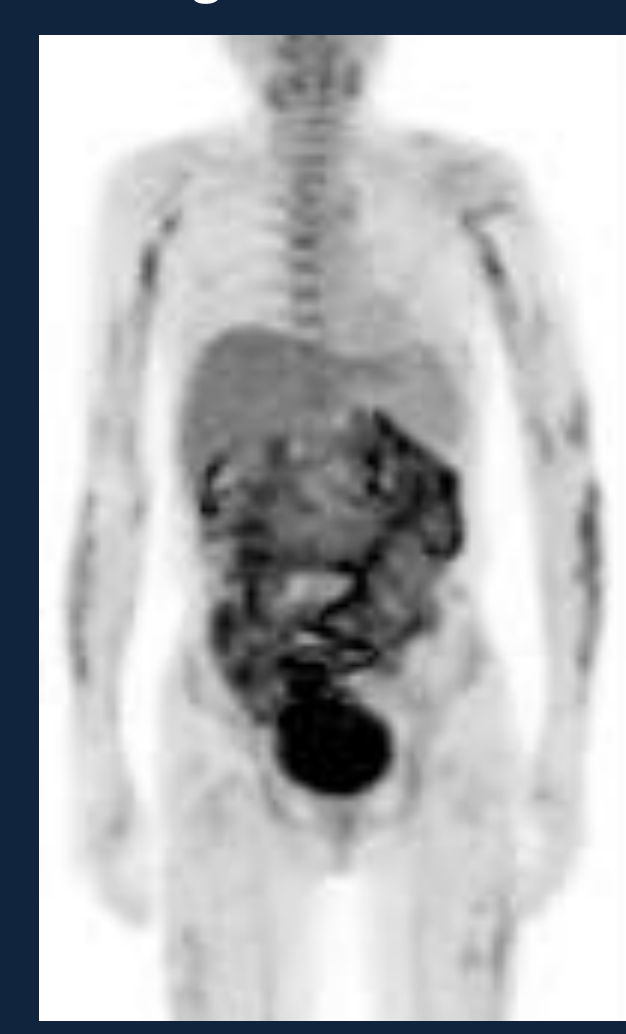

MIP
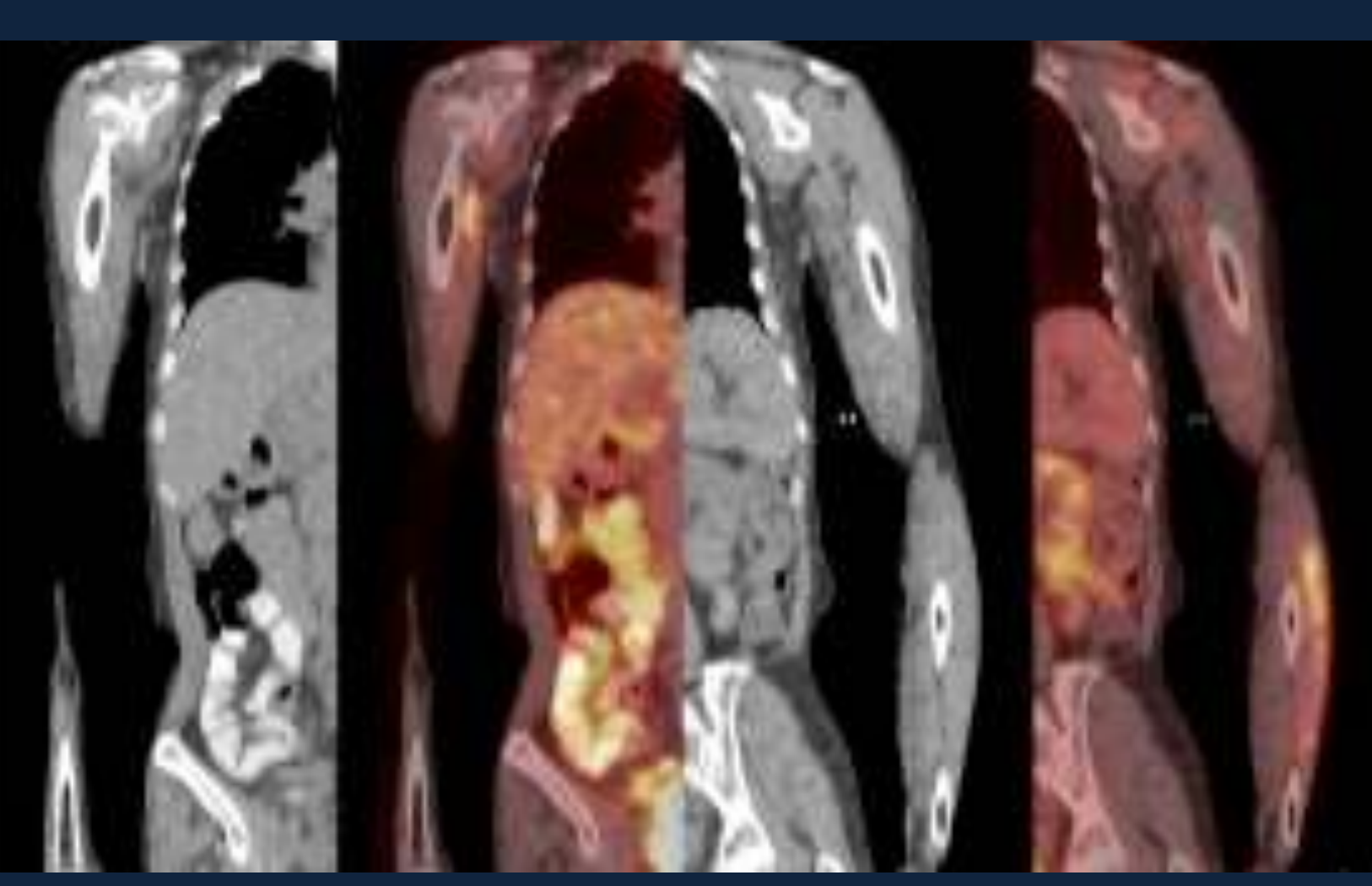

$\mathrm{CT}$ and fusion images

Tc99m-PYP :

- Best assessment of soft tissue $\mathrm{Ca}^{2+}$ burden in juvenile DM (correlates with disease severity and duration) 


\section{Classic Dermatomyositis}

Muscular MR Features

Morphology:

- Fascial $/$ subcutaneous edema = hallmark (precede muscle abnormality)

- Muscle edema ( $\uparrow$ STIR) is predominant

- Mild atrophy ( $\downarrow$ T1)

- Atrophy correlates with duration

Distribution:

- Bilateral symmetric

- Pelvic muscles \& proximal thigh > shoulder girdle

- Rectus femoris and semimembranosus are most commonly involved

- Biceps femoris and vastus medialis most commonly spared

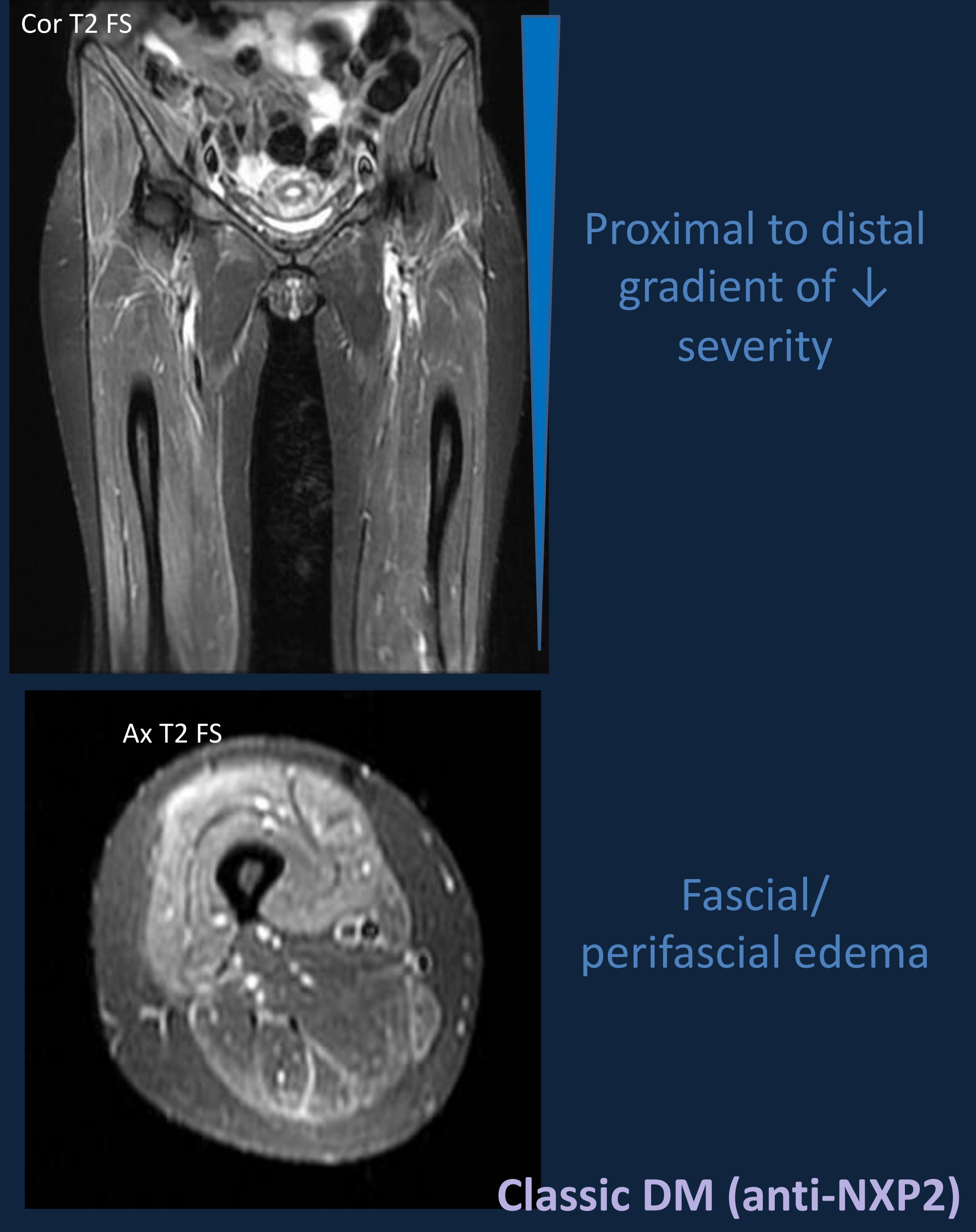




\section{Case: $14 \mathrm{~F}$, respiratory weakness, high CK. Anti- NXP2 +. Diagnosis = Juvenile DM}

August 2015

Axial STIR

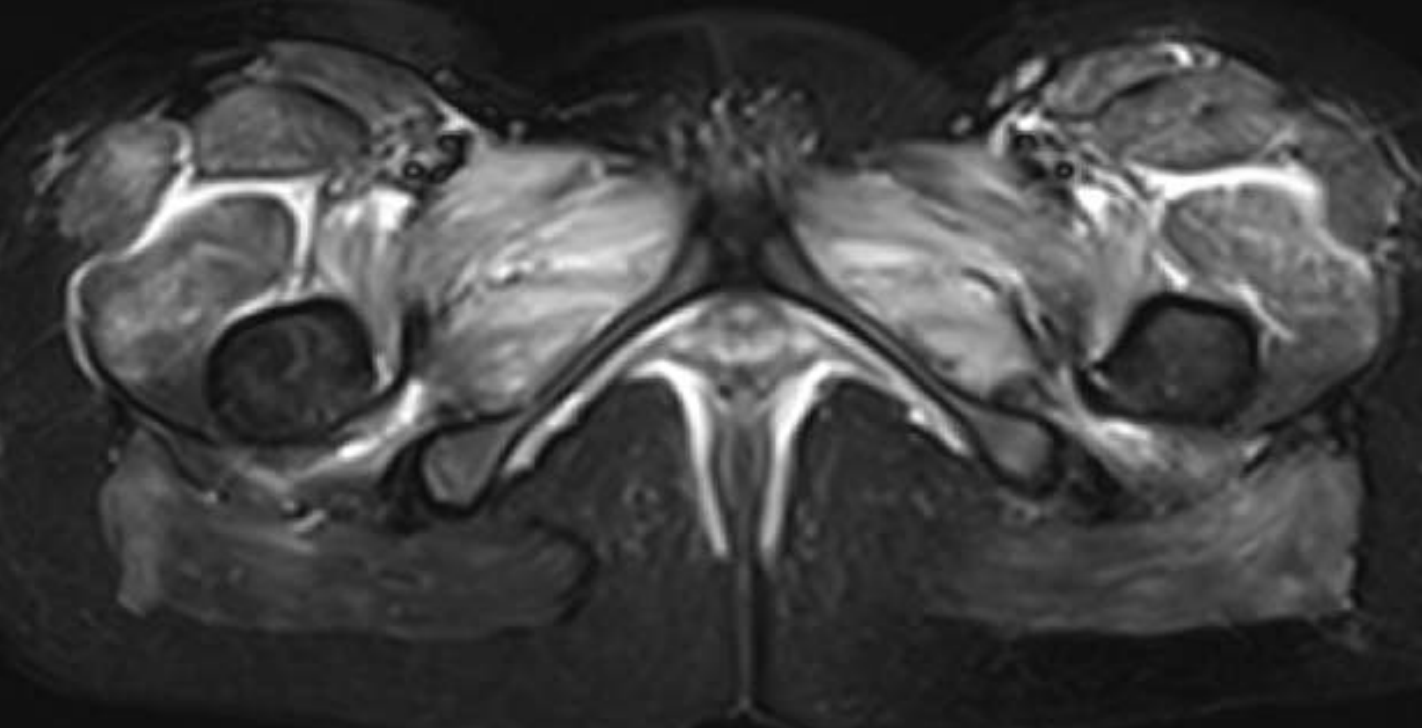

Symmetric pelvic muscles edema

Axial STIR

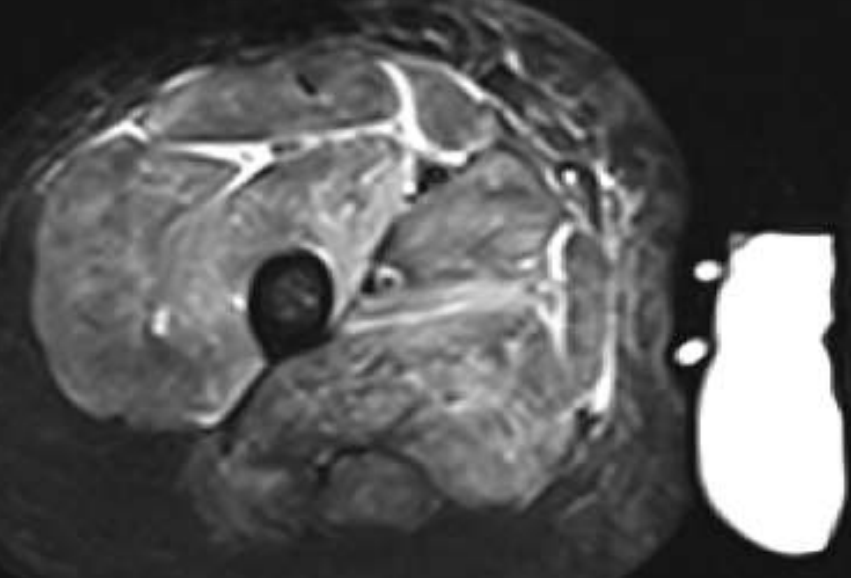

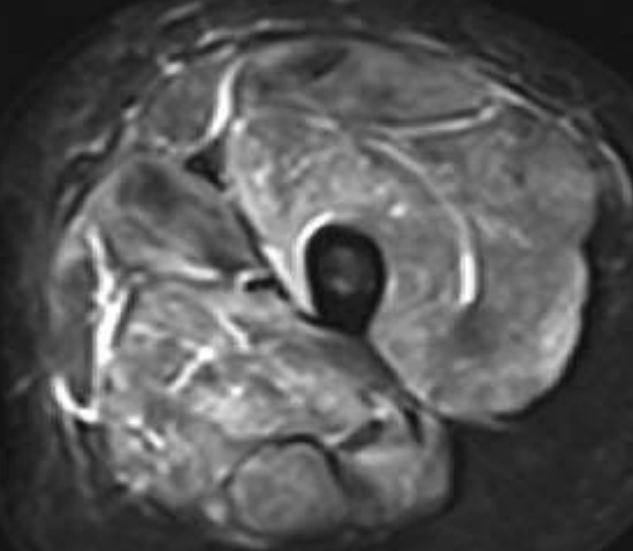

$$
\text { Axial STIR }
$$

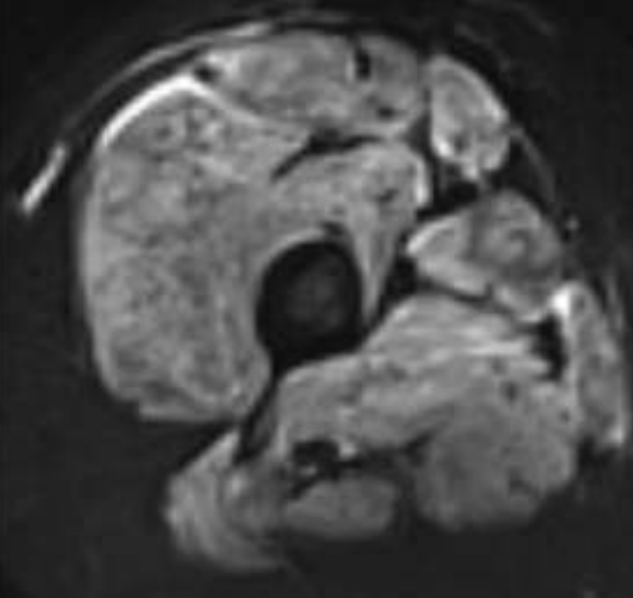

October 2015

Axial STIR
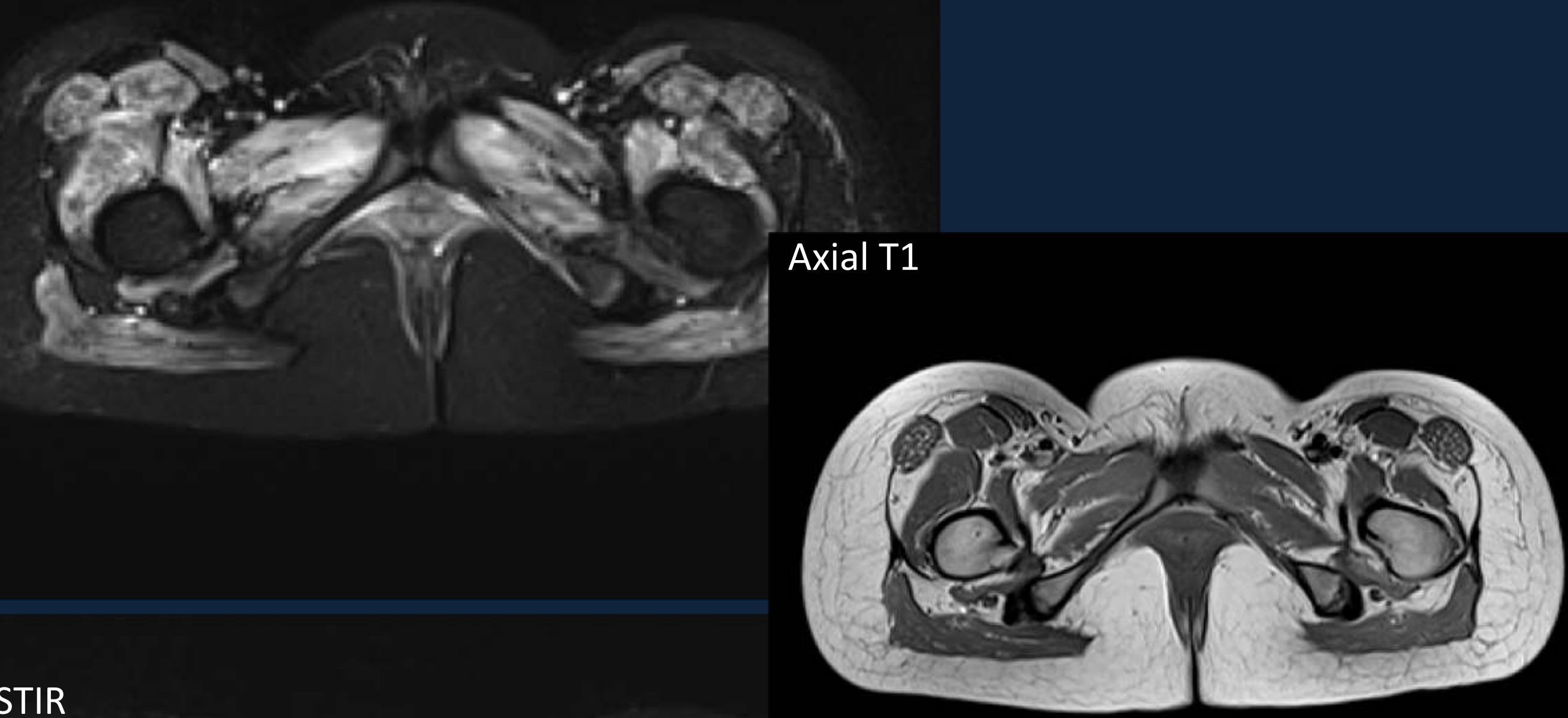

No fatty atrophy 


\section{Fascial Edema in "DM"}

- Inflammation in dermatomyositis

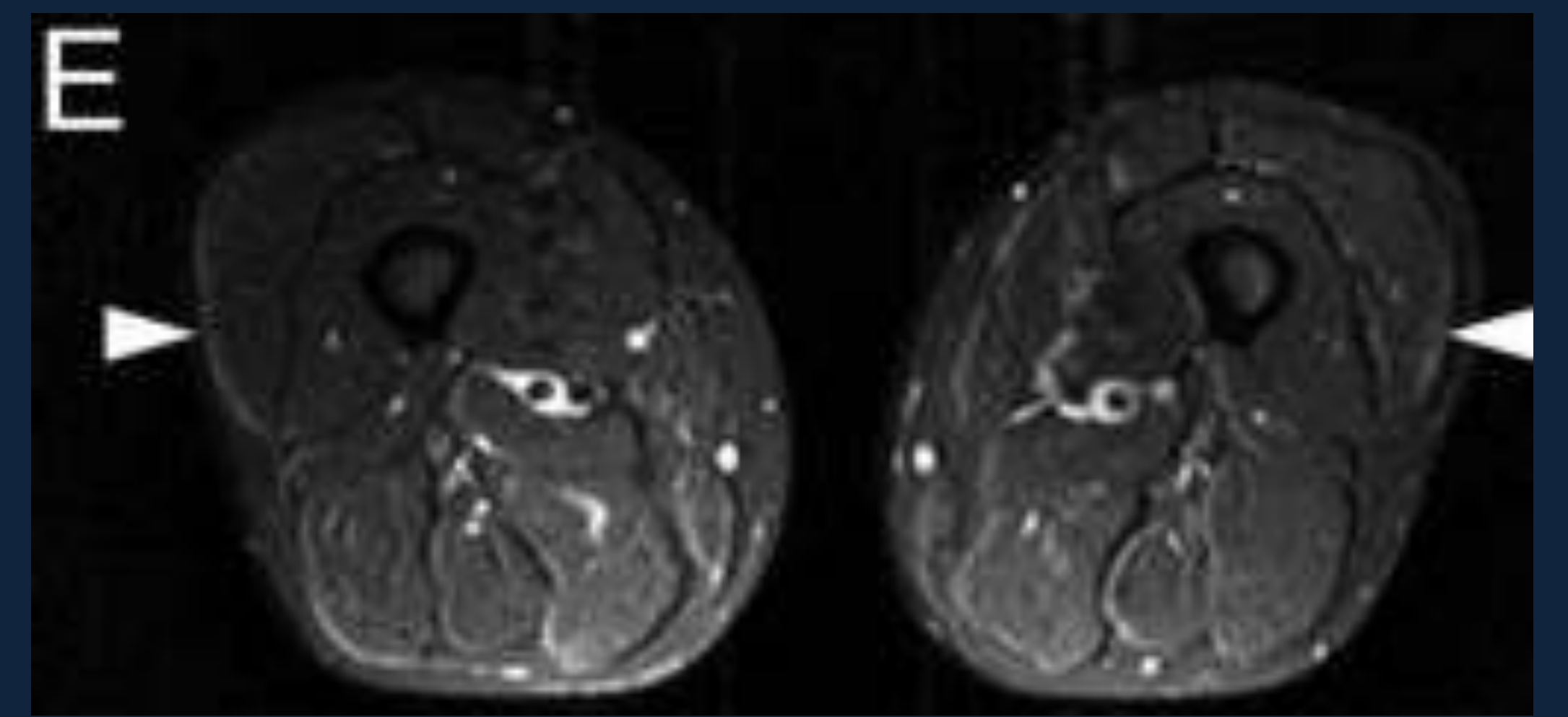

- Fascial edema and peri-fascial muscular edema $\rightarrow$ progresses centrally correlated with findings on en-bloc biopsies

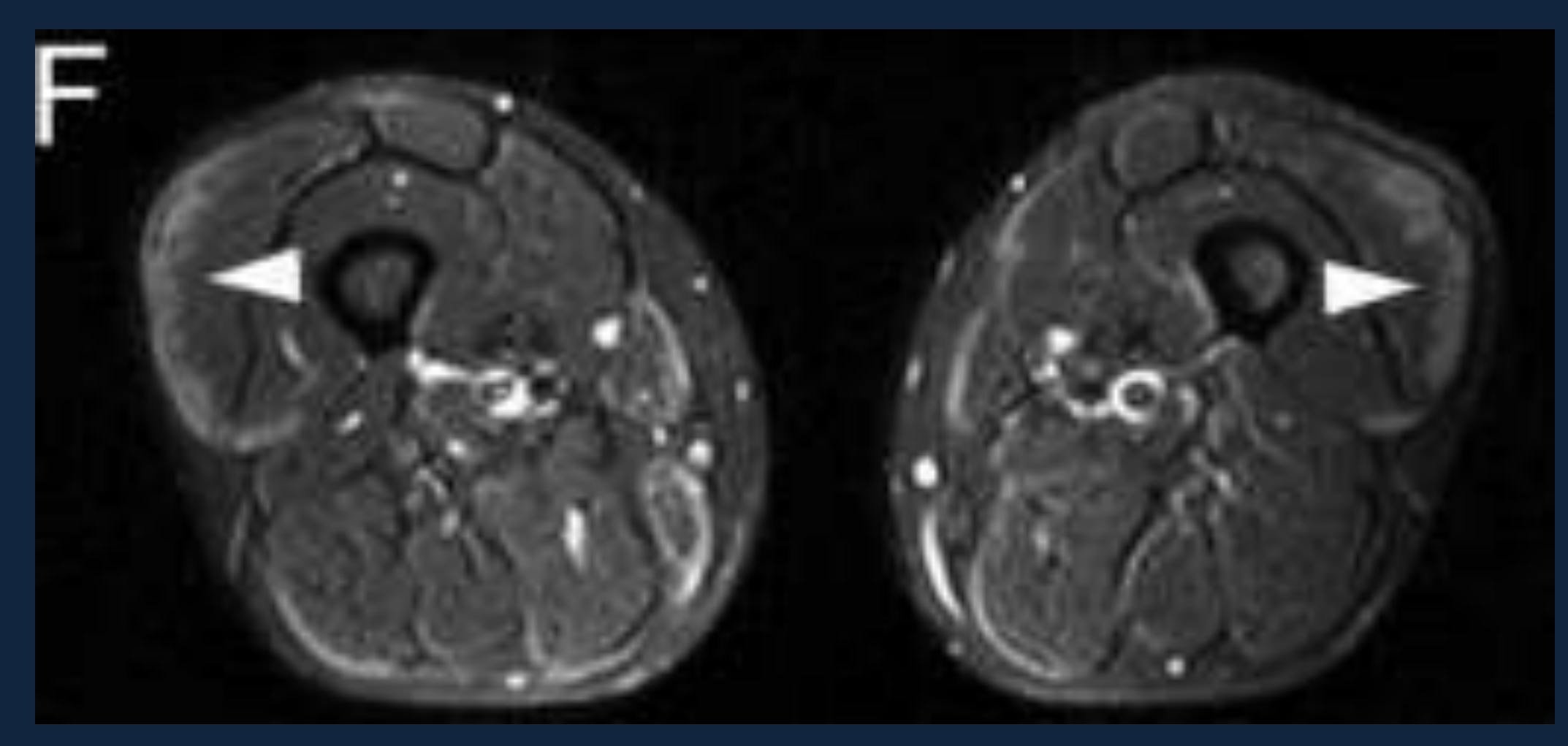

- FDG PET/CT can also demonstrate fascial predominance of radiotracer uptake 


\section{More on Fascial Edema}

Fascial edema is not specific for classic DM as it is also present in various overlap myositis (OM)

- Amyopathic DM (CADM)

- Anti-synthetase syndrome (ASS)

- Scleromyositis (OM-SSc)

- SLE overlap myositis (OMSLE)

- RA overlap myositis (OM-RA)

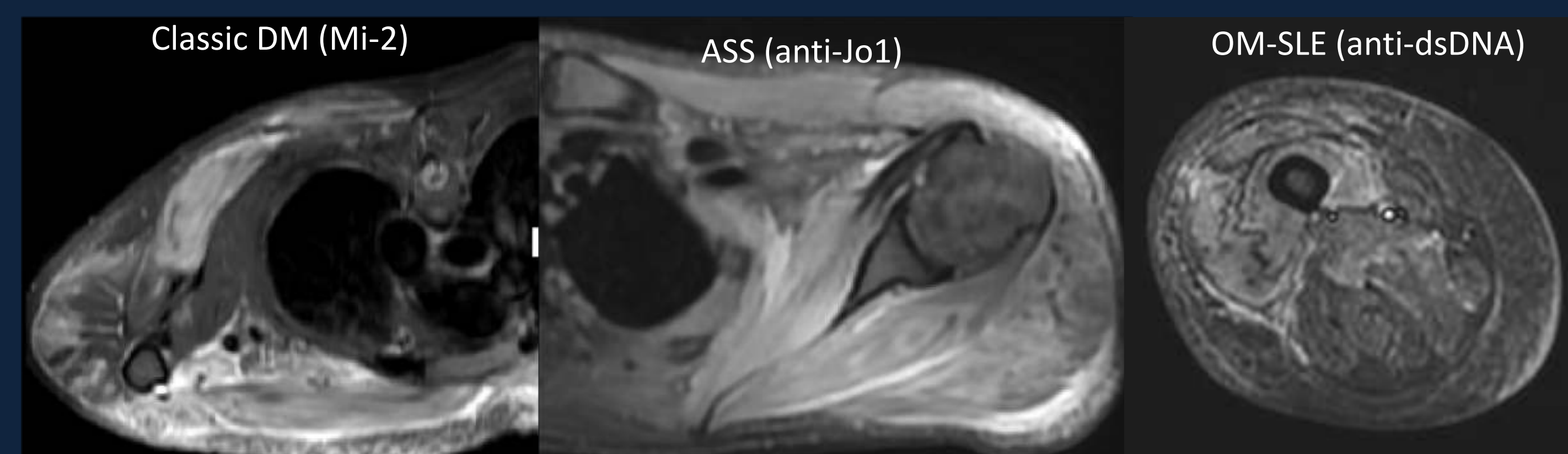

Scleromyositis (anti-Ku \& anti-Scl)

CADM (anti-MDA5)

OM-RA (no Ab)
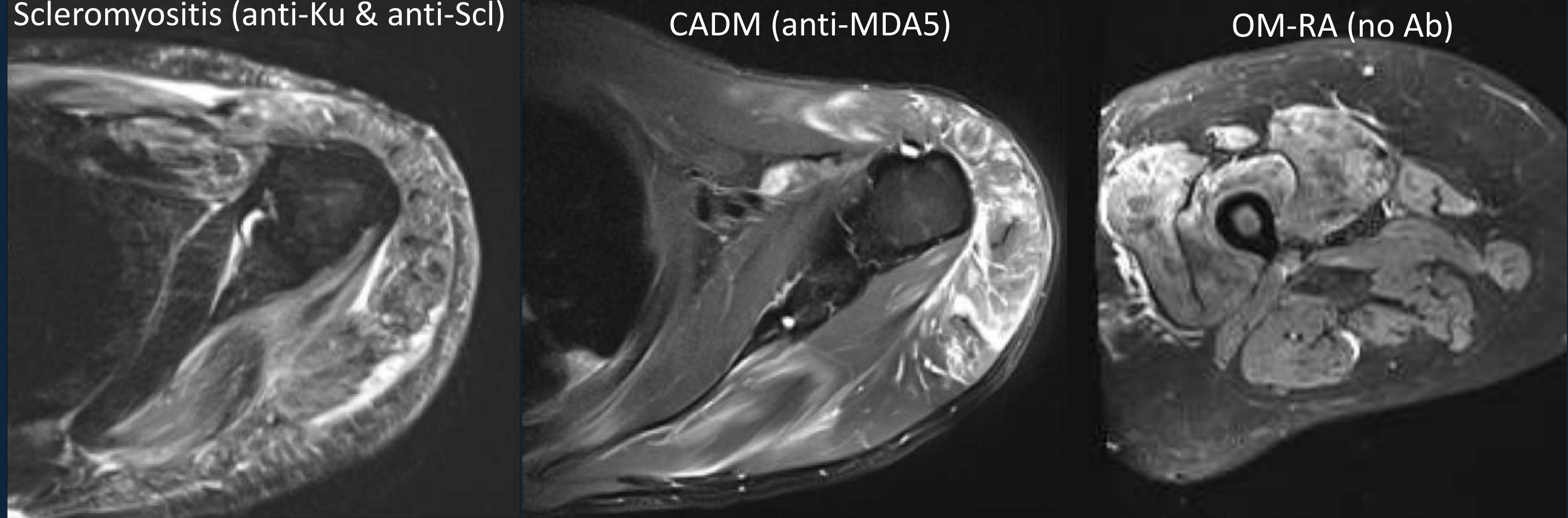

Cm Fascial edema is helpful feature in distinguishing DM or OM from immune mediated necrotizing myopathy (IMNM) and inclusion body myositis (IBM) 


\section{Clinically Amyopathic Dermatomyositis (CADM)}
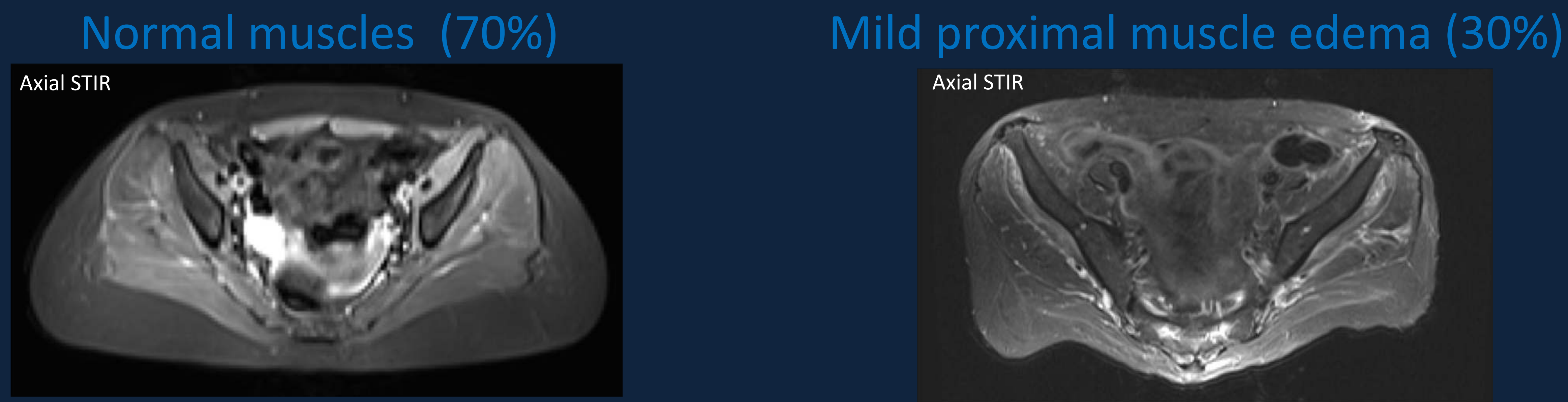

Mild proximal muscle edema (30\%) Axial STIR
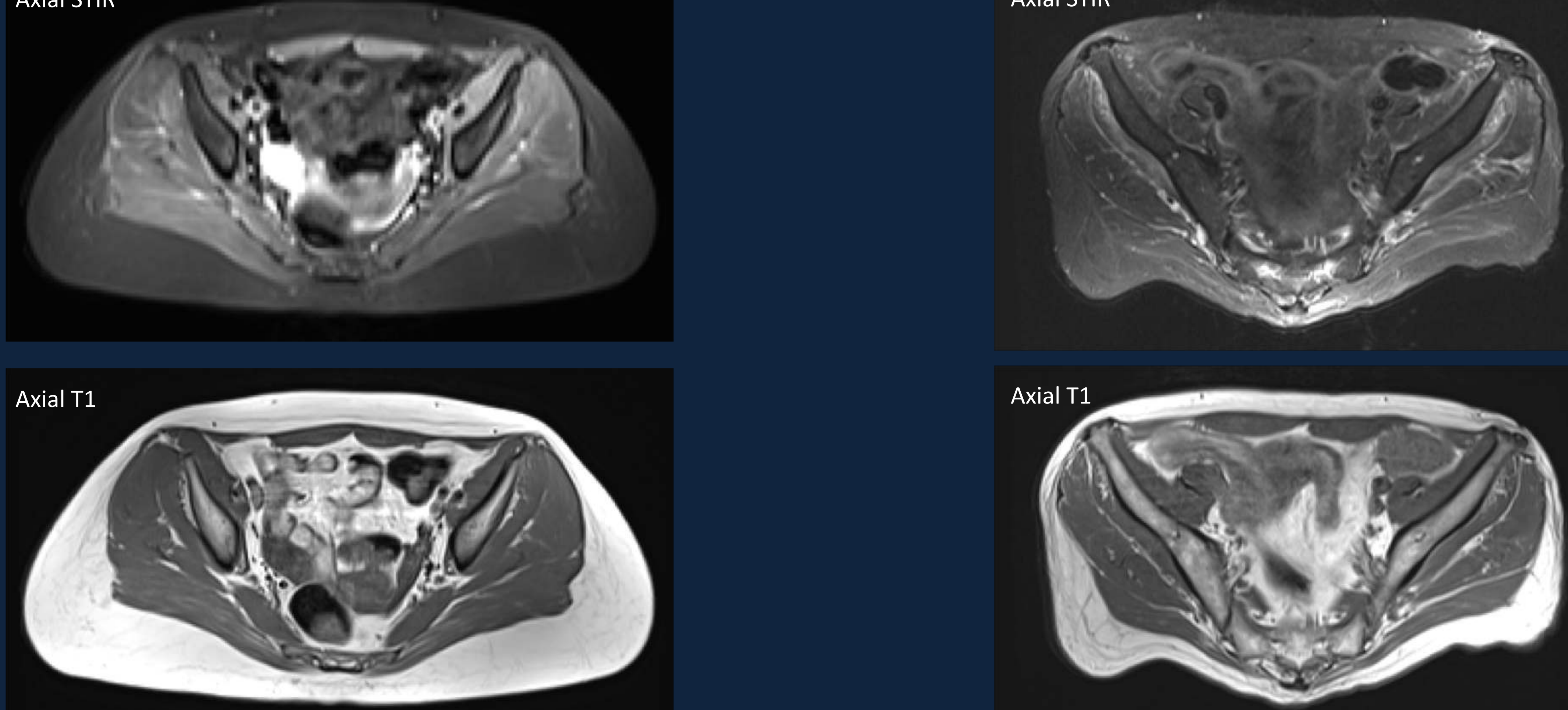

'Clinically' used to describe patients with very mild or no myopathic symptoms but demonstrating myositis on investigations ( $\uparrow \mathrm{CK}$, EMG abnormality, MRI muscle edema) 


\section{Anti-Synthetase Syndrome (ASS)}

Muscle MRI Features

Morphology:

- Fascial and subcutaneous edema similar to classic DM

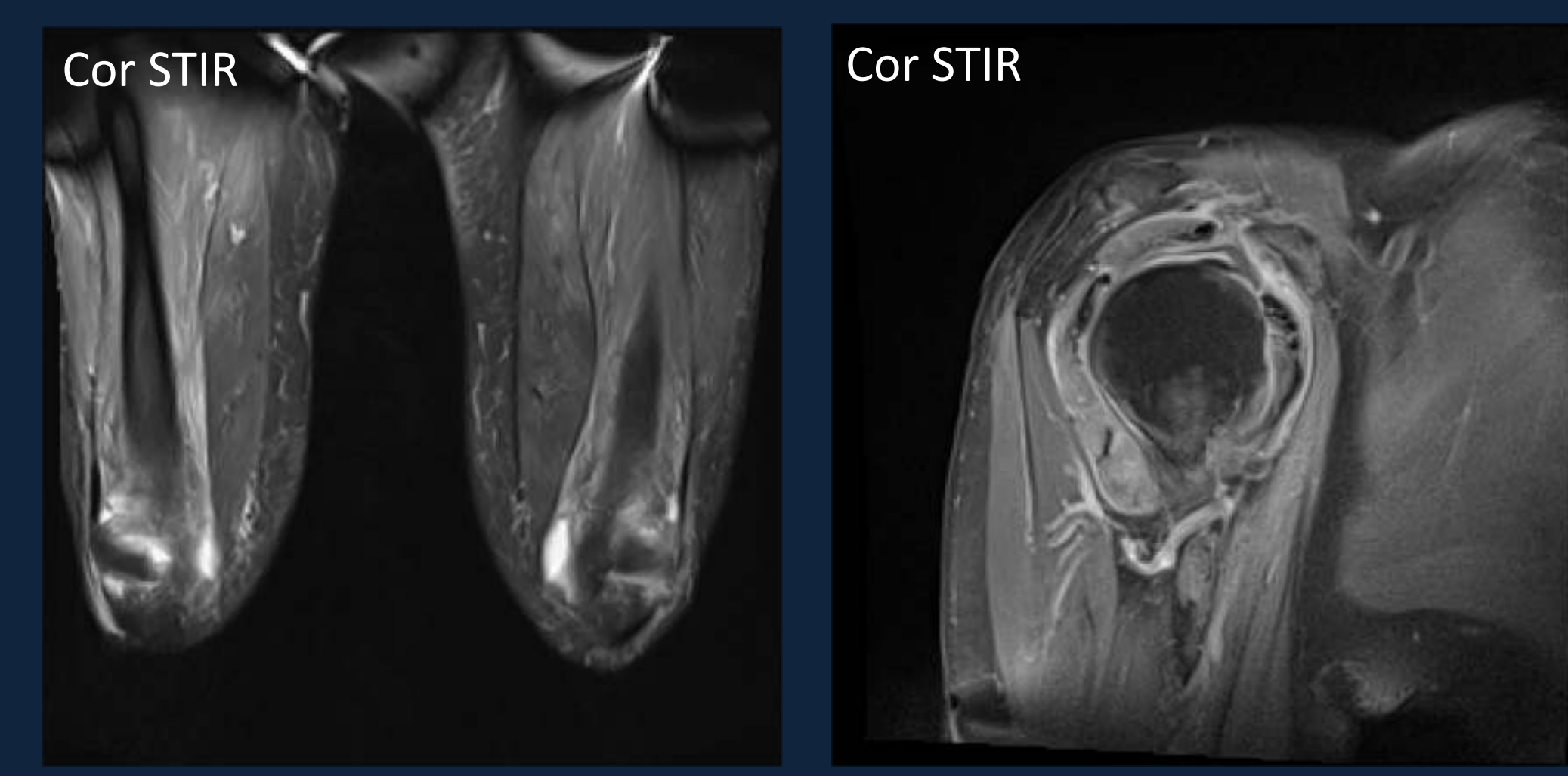

No distal predominance, LE > UE

Distribution:

- Bilateral symmetric

- LE > UE

- Preference for posterior thigh compartment

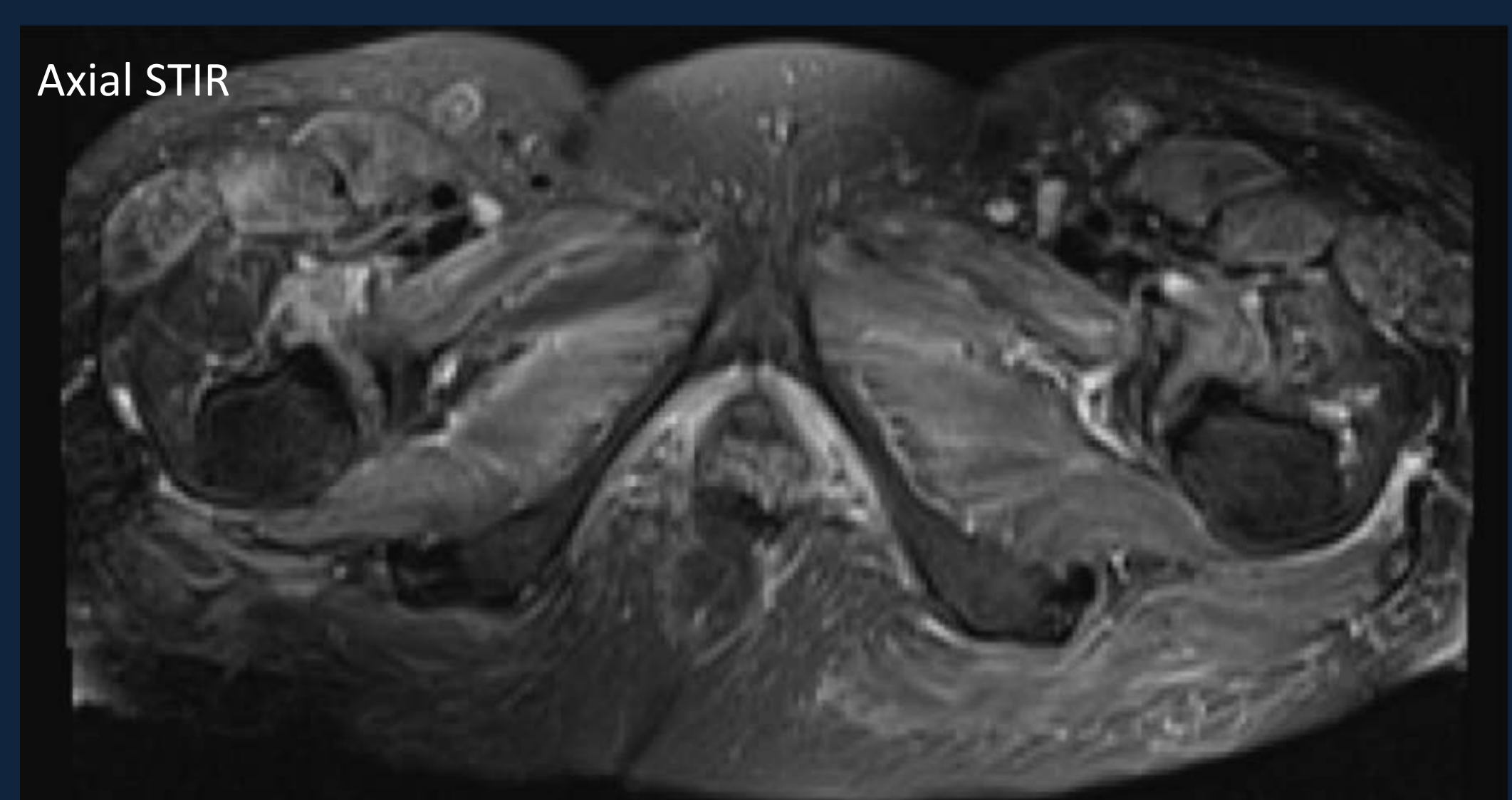




\section{Immune-Mediated Necrotizing Myopathy (IMNM)}

\section{Muscle MRI Features}

- Anti-SRP associated with more severe muscle involvement

- Fatty infiltration and atrophy > edema

- Distribution:

- Lateral rotators, glutei, medial and lateral compartments

- Adductor brevis and obturator externus = most common

- More extensive and asymmetric compared to $\mathrm{DM} / \mathrm{OM}$

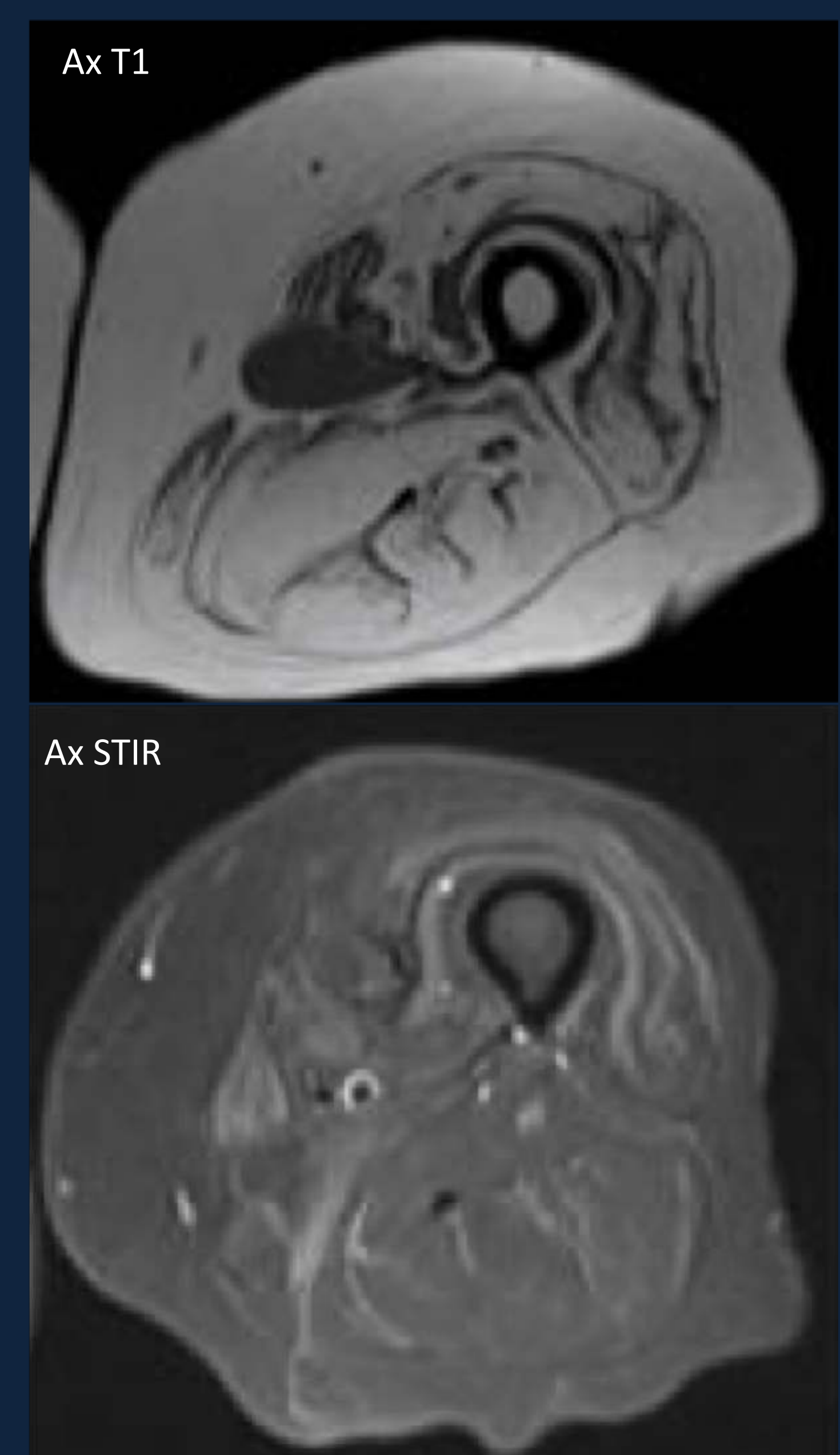




\section{Scleromyositis}

\section{Muscle MRI Features}

Morphology:

- Similar to that observed in classic DM and ASS

- Subcutaneous edema \& fascial thickening/edema (89\%)

- Abnormal muscle signal on STIR (78\%)

Distribution: key feature to favour the diagnosis!

- truly generalized pattern present (50\%)

- lower leg predominance in $44 \%$

- bilateral symmetric

Extra-muscular findings are important

- presence of enthesitis (56\%)

- tenosynovitis (61\%) 


\section{Inclusion Body Myositis (IBM)}

Muscle MRI Features

\section{Morphology}

- Predominantly muscle volume loss and fatty infiltration ( $\uparrow \mathrm{T} 1$ )

- Mild edema ( $\uparrow$ STIR)

\section{Distribution}

- Bilateral, asymmetric, LE > UE

- LE:

- Sparing of rectus femoris (75\%)

- Undulating fascia sign due to vastus lateralis and intermedius atrophy

- Proximal to distal gradient of more severe involvement

- Anterior > posterior or medial thigh compartments

- UE: Flexor digitorum profundus (FDP) often demonstrates fatty infiltration in isolation

\section{6}
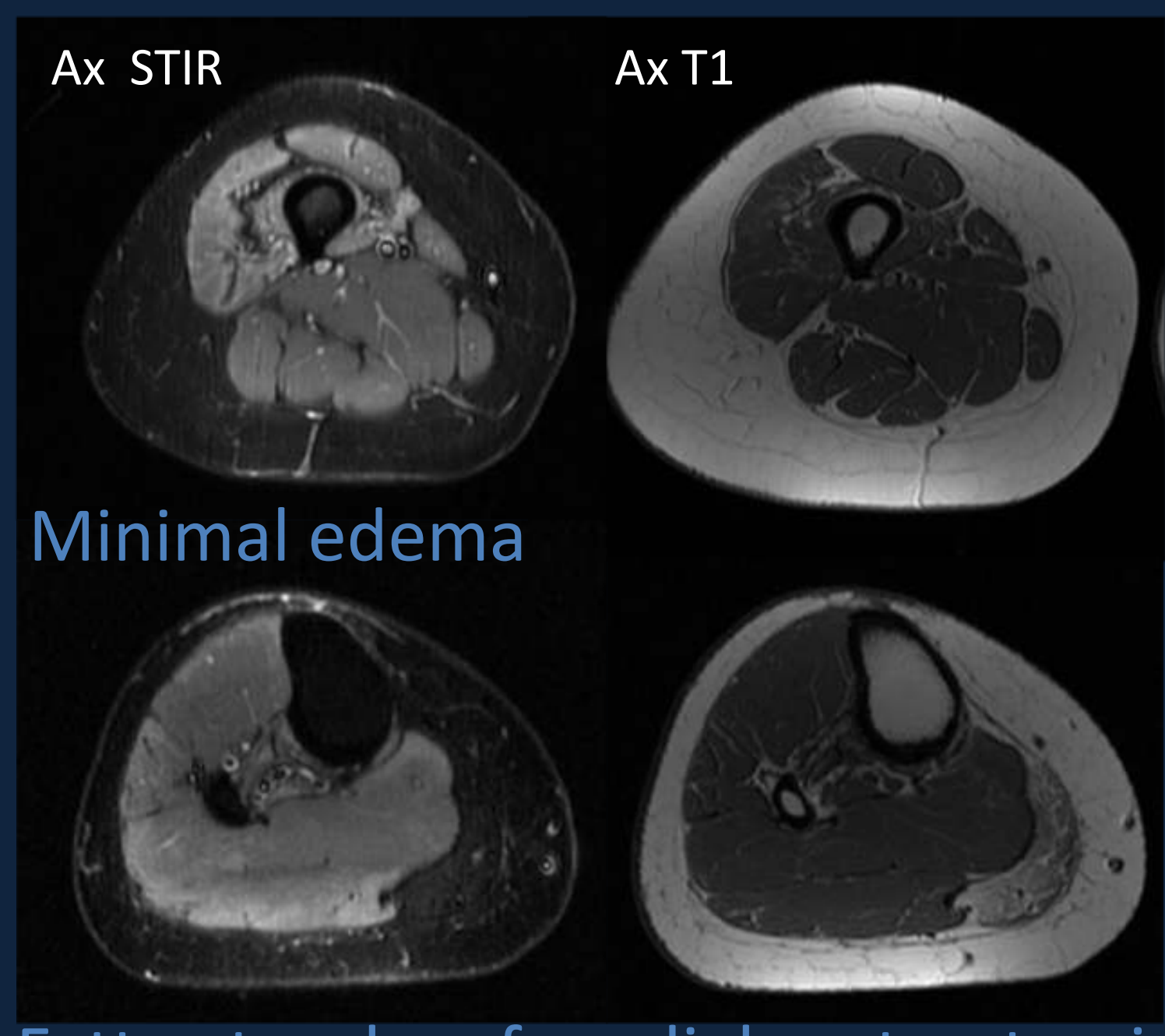

Fatty atrophy of medial gastroctemius

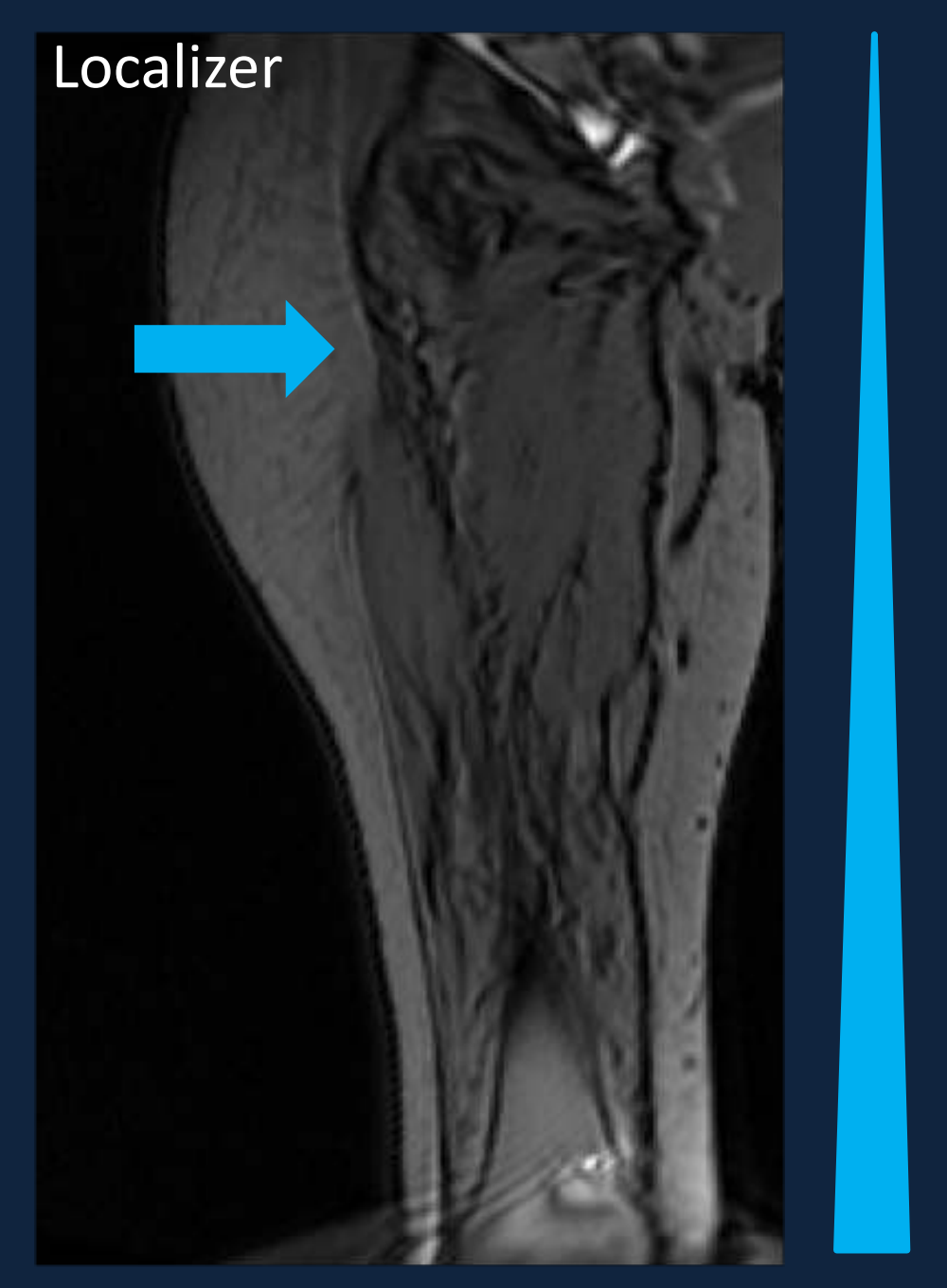




\section{Imaging in Idiopathic Inflammatory Myopathies (IIM)}

\section{MSK Imaǵing}

- Muscular/fascial involvement

- Soft tissue calcinosis

- Arthropathies 


\section{$\mathrm{XR}$}

Predominant role in the evaluation for calcinosis

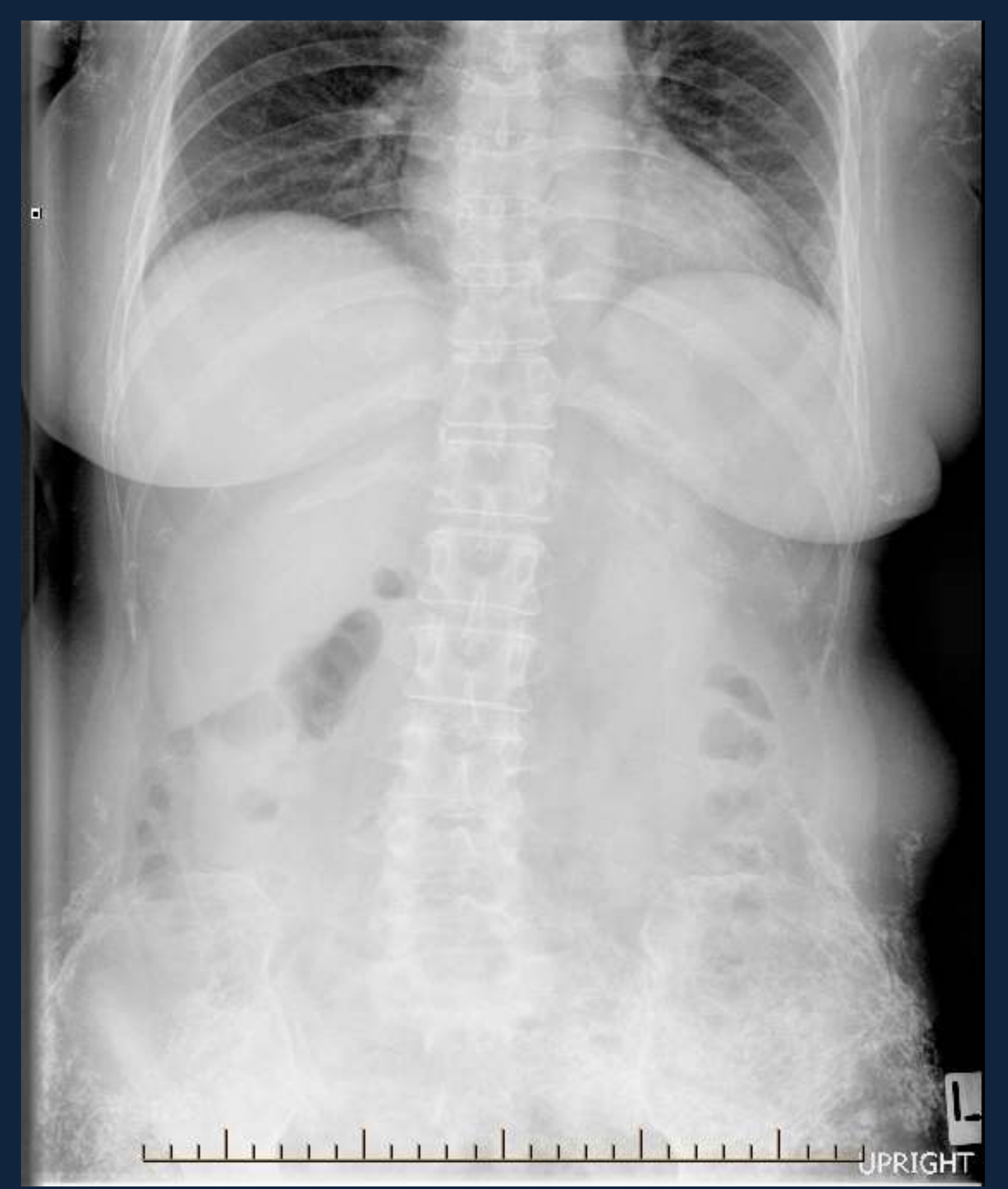

Abdominal XR in a DM patient with soft tissue calcinosis

\section{CT}

Increasing interest for the use of dual energy $C T$ (DECT) for the evaluation of calcinosis

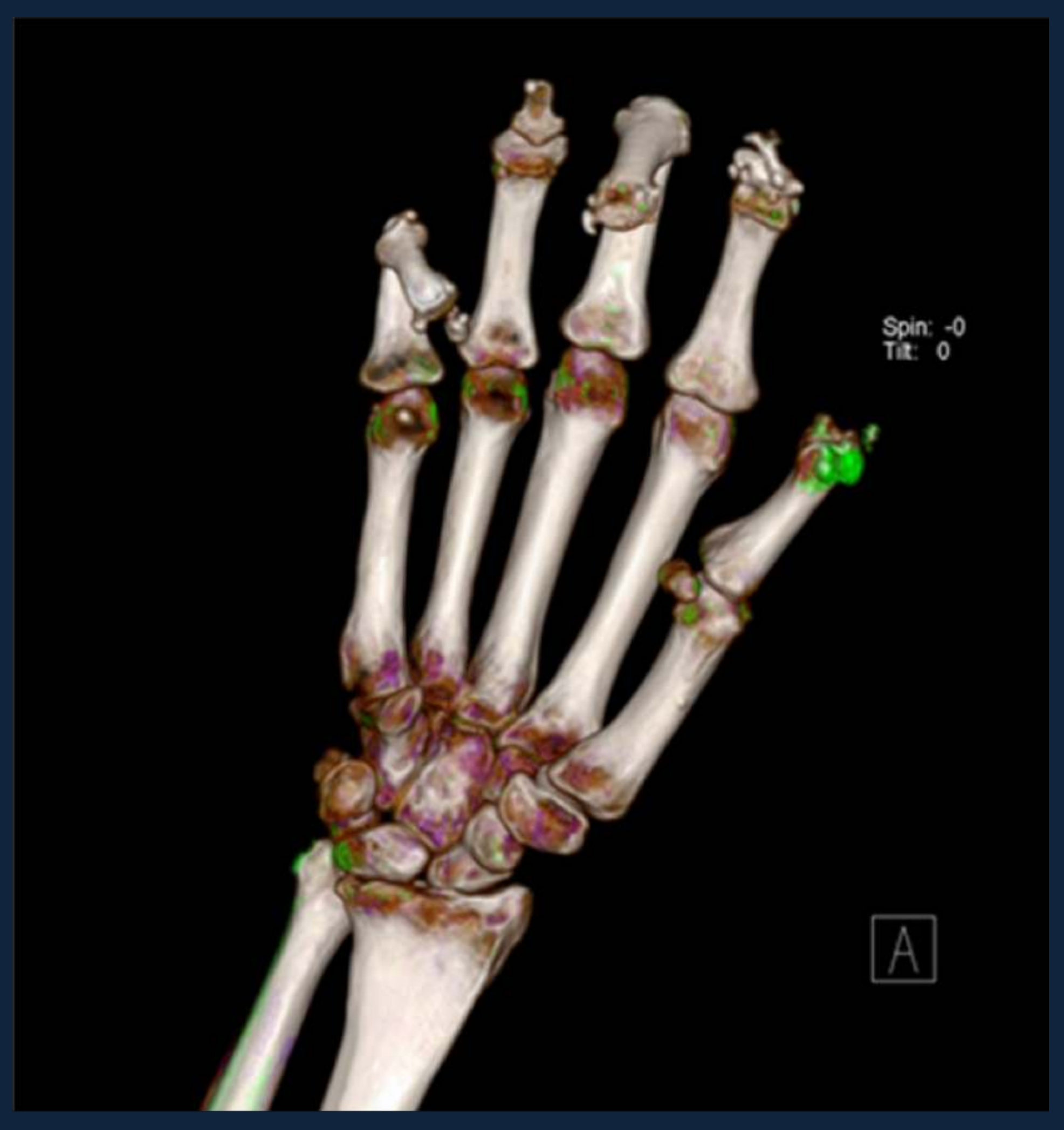

Adapted from Hsu et al. 


\section{Soft Tissue Calcifications (Calcinosis Cutis)}

- Pathomechanism: chronic tissue injury, $\uparrow$ intracellular $\mathrm{Ca} / \mathrm{PO} 4, \downarrow$ concentration / effectiveness of calcification inhibitors

- Predominantly in juvenile dermatomyositis (JDM)

- $44-70 \%$ (JDM) vs. $11-20 \%$ (adult onset DM)

- Scleromyositis and OM-MCTD demonstrate calcifications classically associated with scleroderma

- Significant morbidity (pain, $\downarrow$ mobility, joint contracture, ulceration and infection) and difficult to treat

- Serologic correlation:

- Anti-NXP2 has OR 4 (JDM) and OR 1.62 (adult DM)

- Anti-TIF1g is protective from calcinosis (OR 0.2)

- Anti-PM/Scl (39\%) and anti-U1-RNP are highly associated with calcinosis

- Anti-MDA5 may also demonstrate calcifications

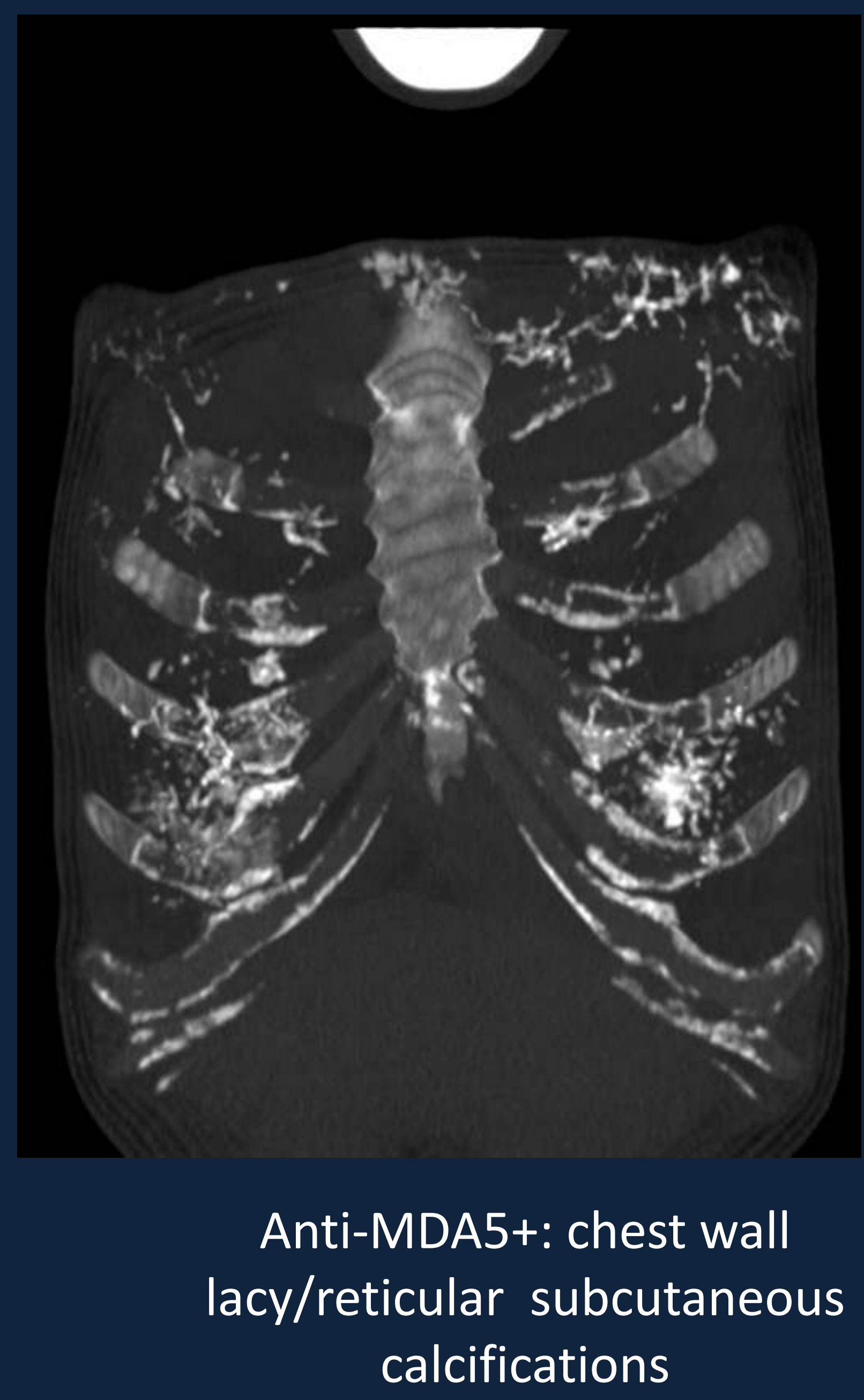




\section{Patterns of Calcifications}

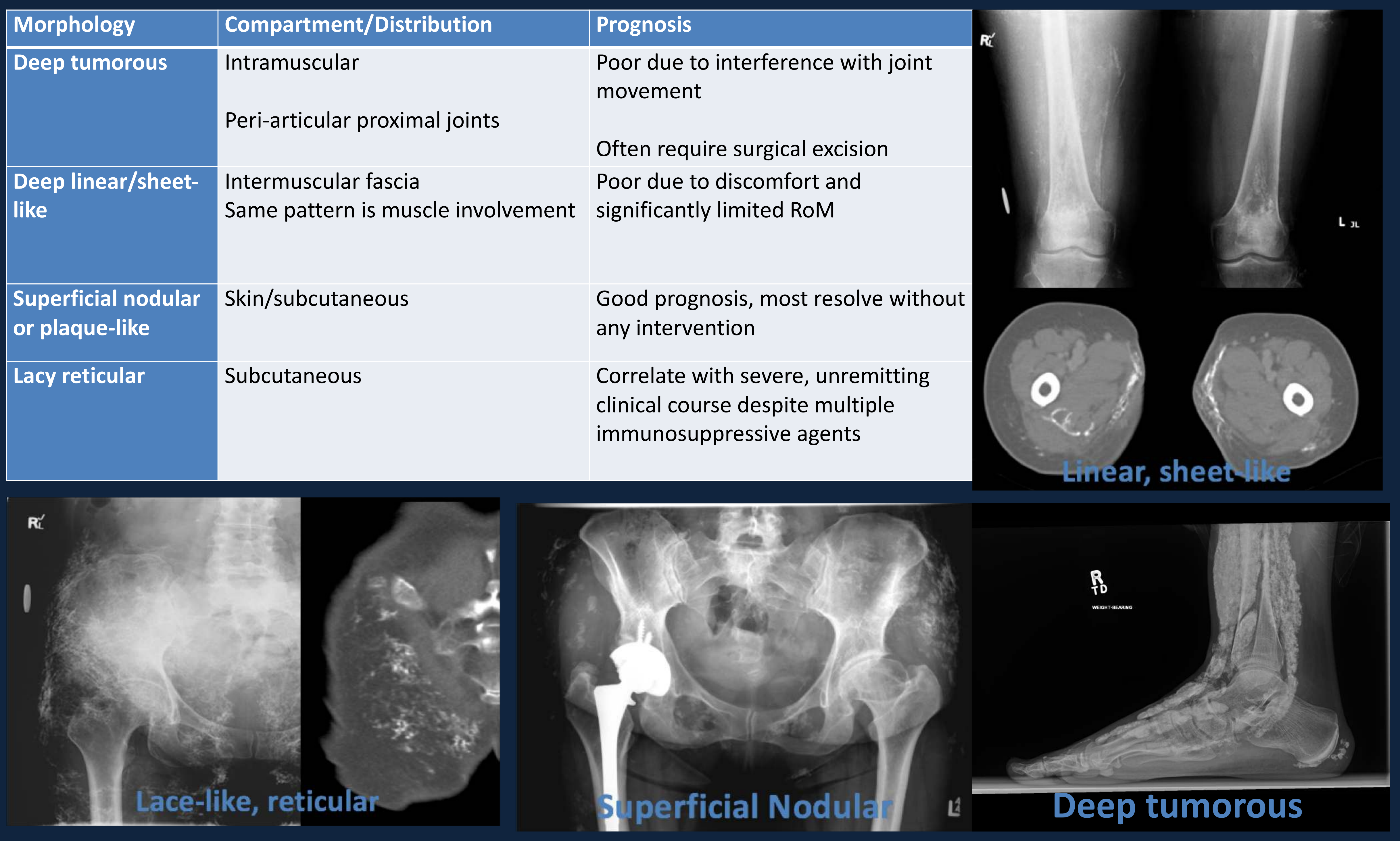

MSK 


\section{Cases}

Classic DM (Anti-NXP2)

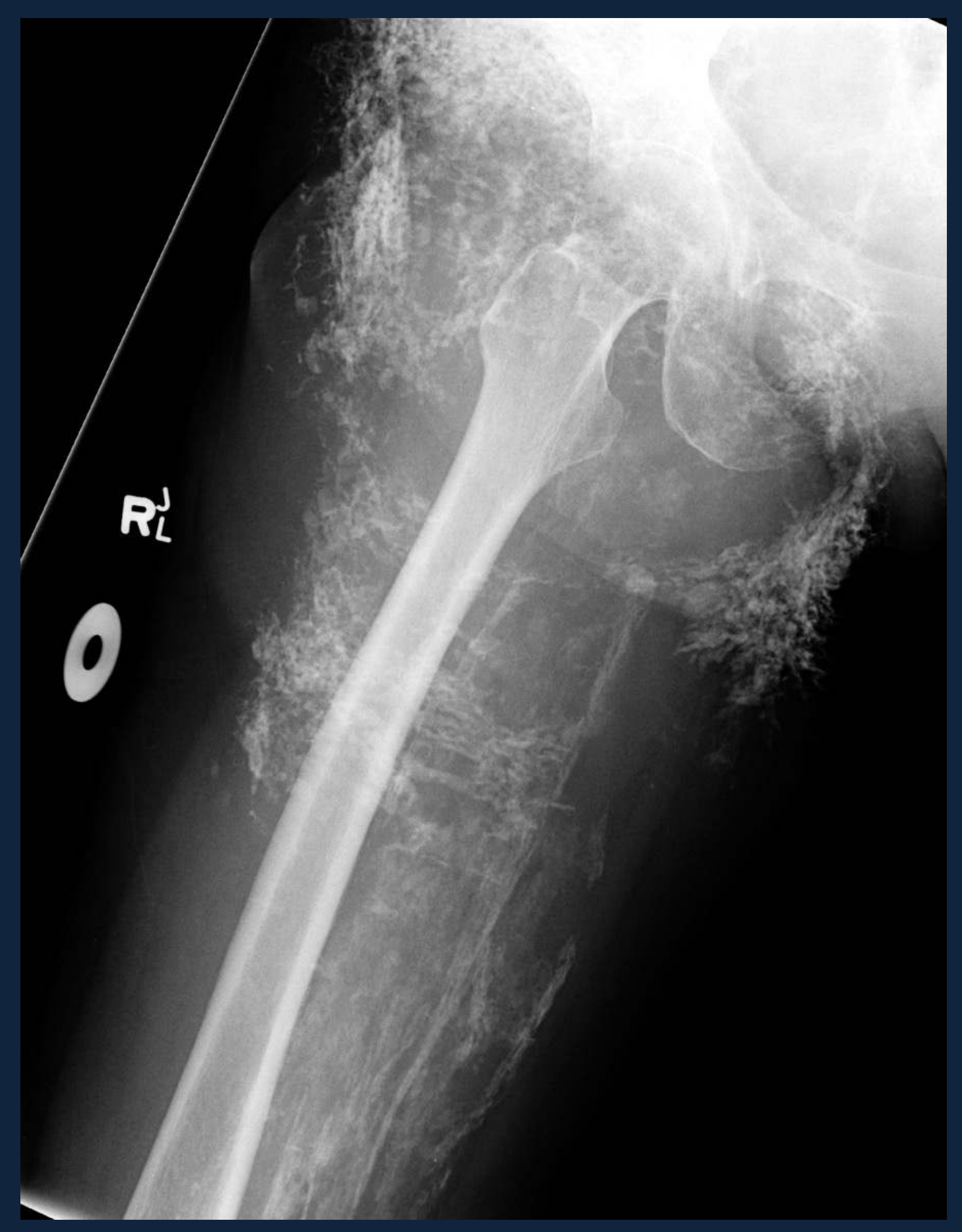

Lace-like reticular subcutaneous \& sheet-like fascial calcifications
Scleromyositis (seroneg)

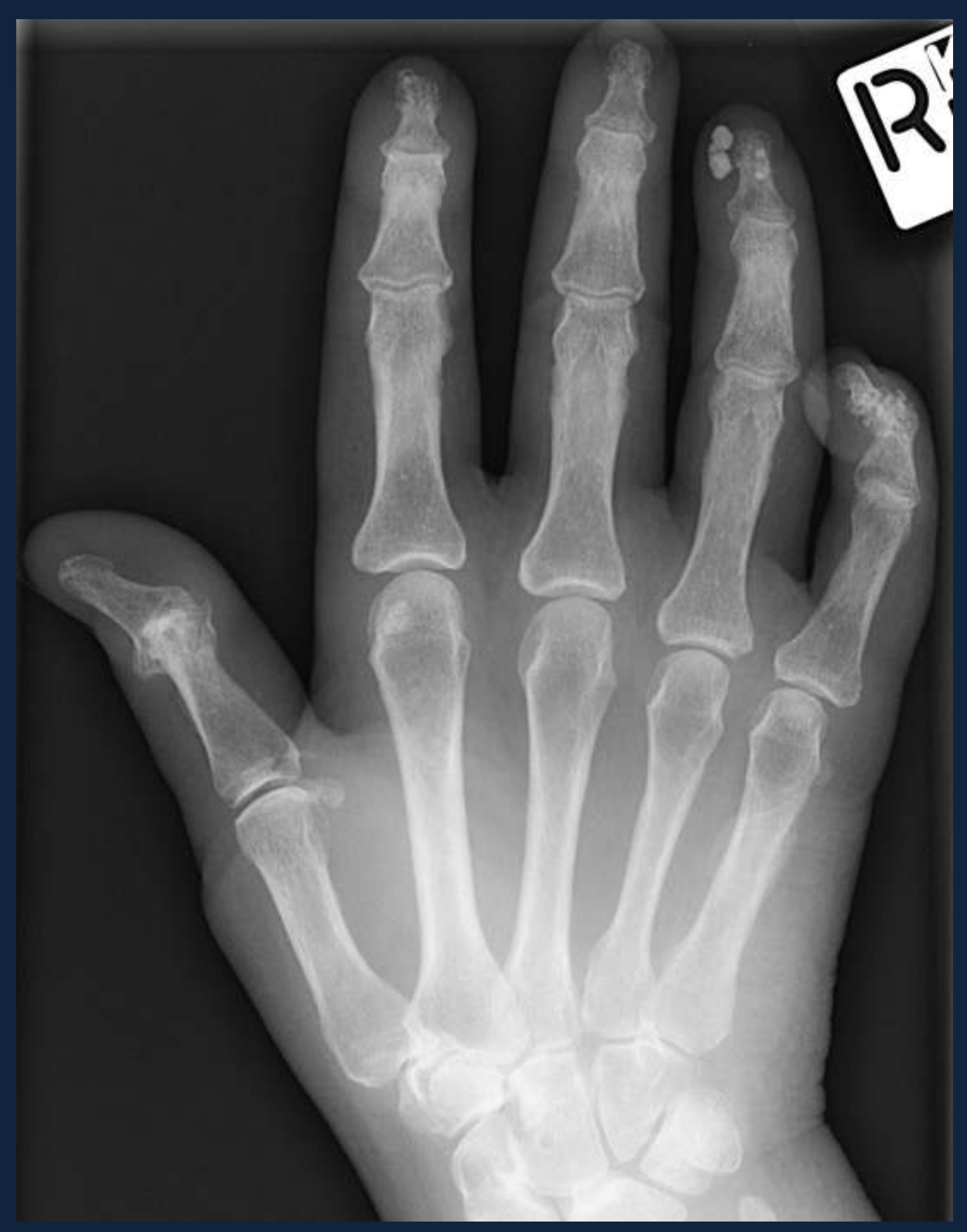

Small, smoothly lobulated calcifications in peri-articular distribution involving the hand
Scleromyositis (anti-PM/Scl)

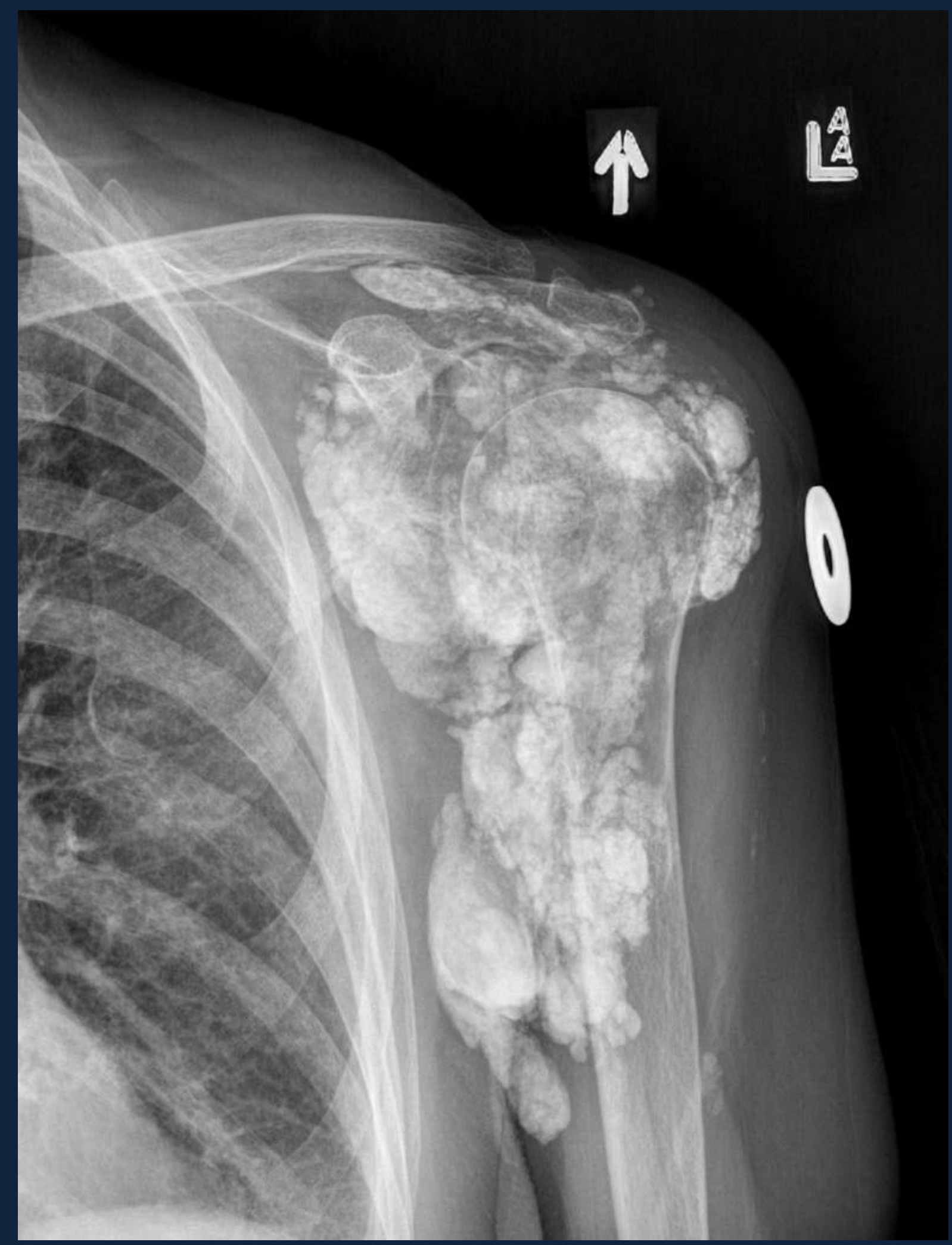

Massive, peri-articular lobulated calcifications 


\section{Imaging in Idiopathic Inflammatory Myopathies (IIM)}

\section{MSK Imaging}

- Muscular/fascial involvement

- Soft tissue calcinosis

- Arthropathies 


\section{Anti-Synthetase Syndrome (ASS)}

\section{Background}

- $27 \%$ of patients present with polyarthritis with a delay of myositis and ILD

- Polyarthritis = key component of the 'FIRMA'

\section{Forms of Arthritis}

- Distal symmetric polyarthritis mimicking RA distribution +/- erosions (most common)

- Subluxing arthropathy (ie. floppy thumb syndrome)

- Polyarthralgia w/o radiographic abnormality

\section{Serologic Correlation}

- Radiographic abnormal arthropathy most prevalent in anti-Jo-1

- Clinical diagnosis of polyarthritis equal in all ASS serologies
Clinical features:

Fever

ILD

Raynaud's

Mechanic's hands

Arthritis : RA pattern, subluxing or swelling

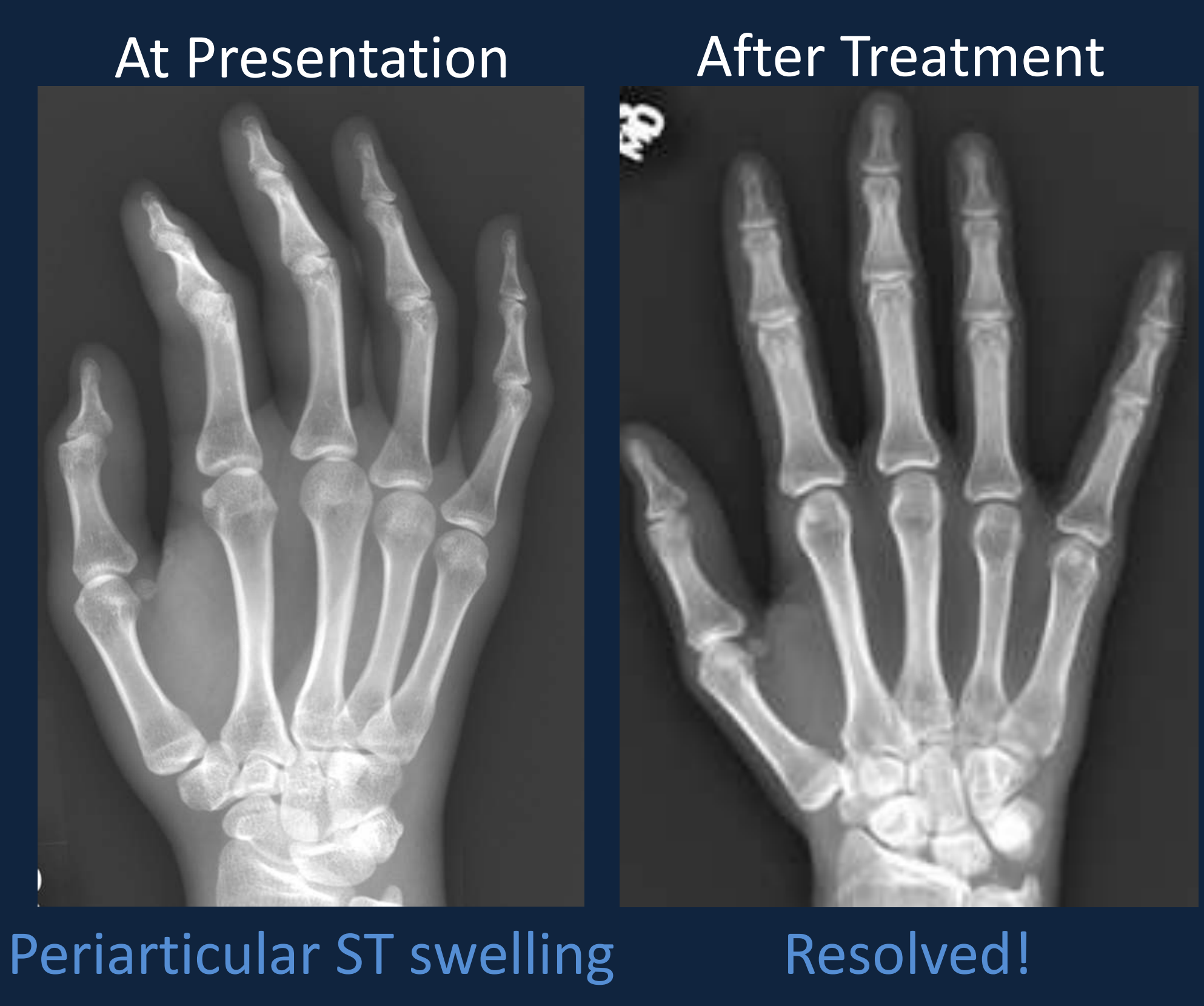




\section{Subluxing Arthropathy in ASS}

Clinical Features:

- Floppy thumb syndrome

- More common in prolonged diseased duration

Radiographic Features:

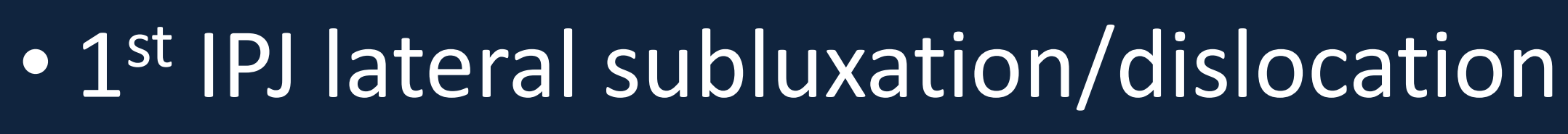
before other joint malalignment

- MCP subluxation less prominent than DIPJ (opposite to Jaccoud's)

- DIP erosions predominant

- Elbow/shoulder/ankle/knee erosions occur but subluxation limited to hands

- Periarticular $\mathrm{Ca}^{2+}$, predominantly DIPJ

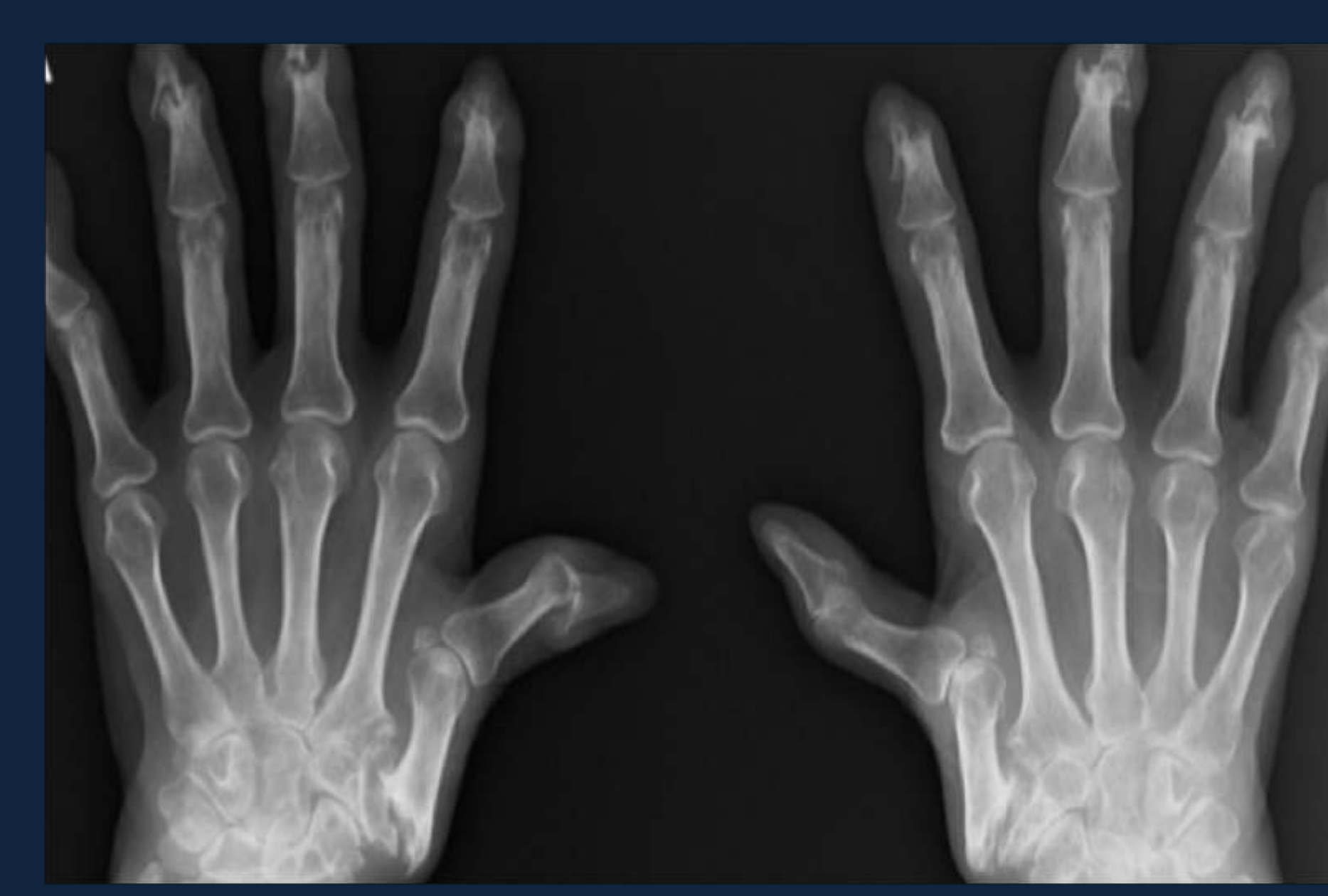

Adapted from Kaneko et al. Rheumatology, 53 (6) 2014

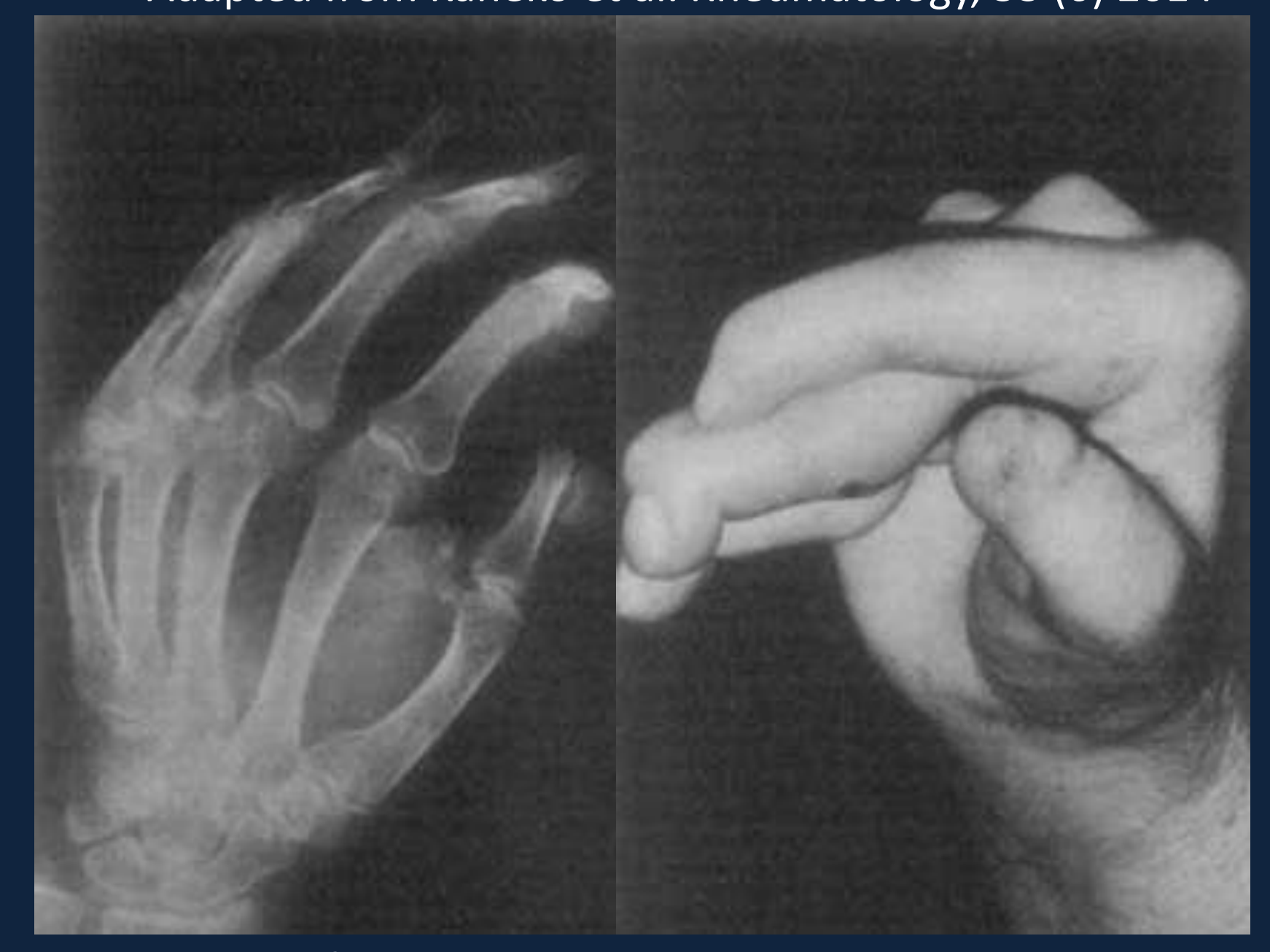


Case: 56F, history of "dermatomyositis" presentation with arthritis

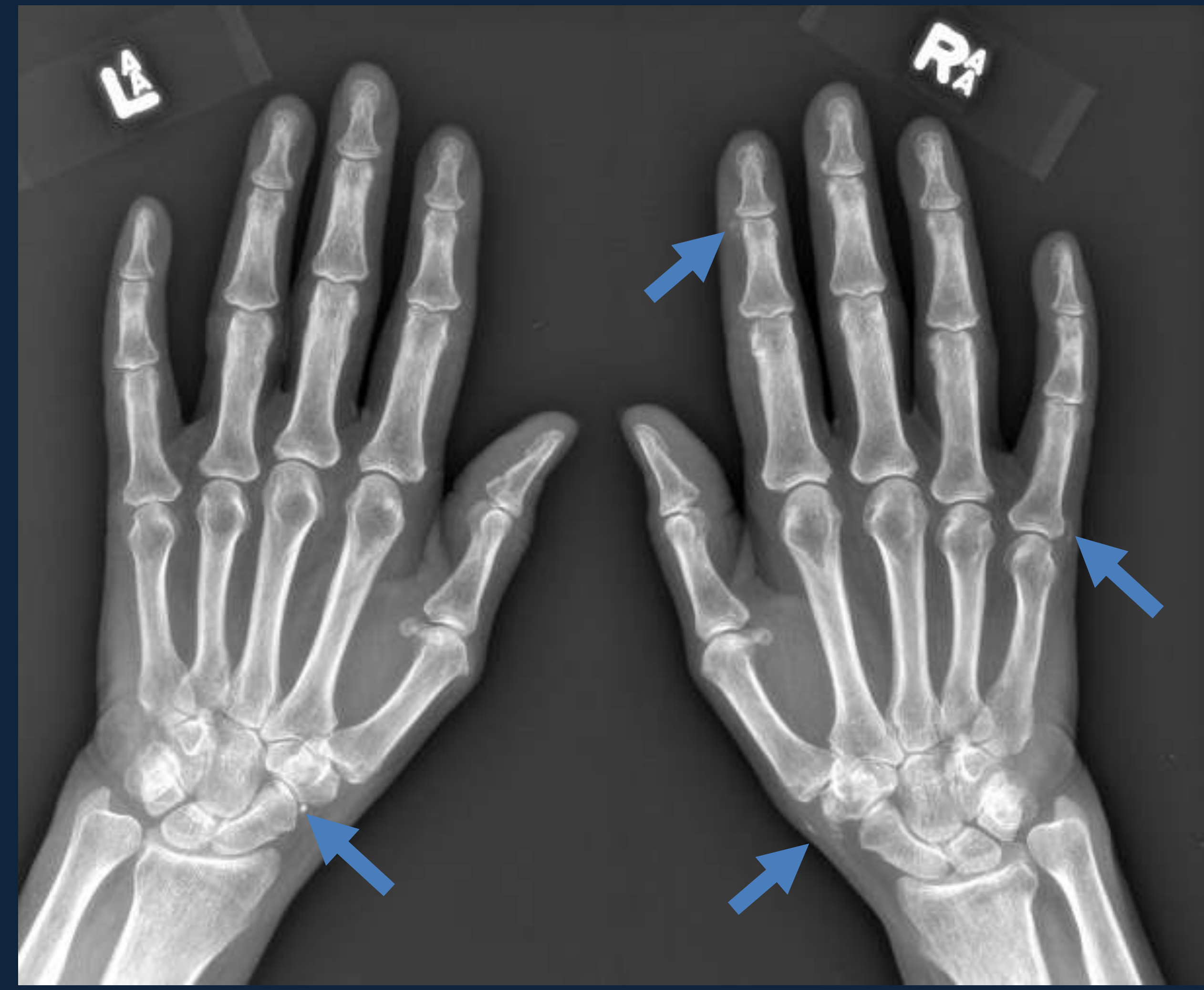

$\rightarrow$ Peri-articular calcifications

$\Rightarrow$ Subluxation
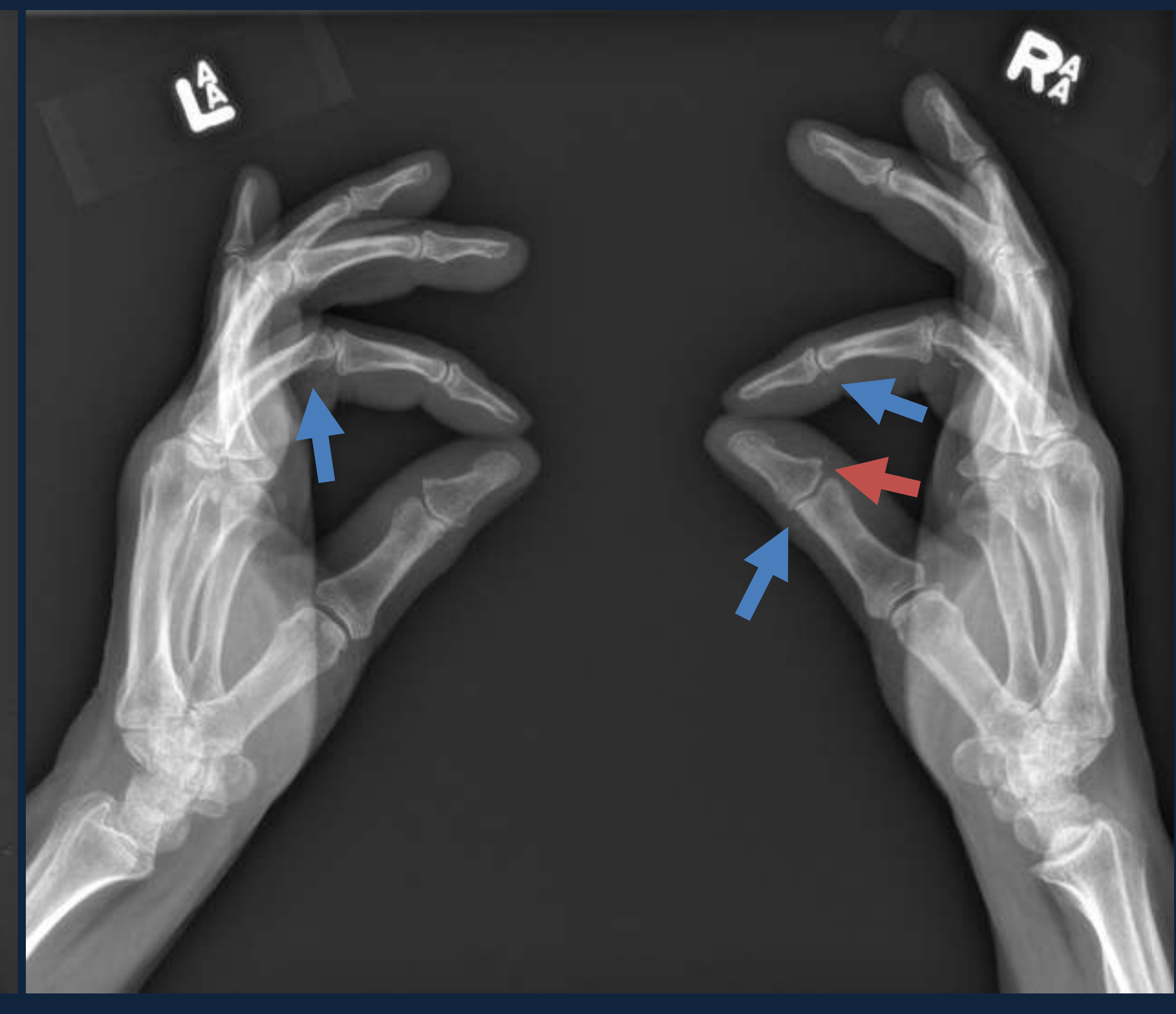

Diagnosis: Anti-synthetase Syndrome (anti-Jo-1) 
Imaging in Idiopathic Inflammatory Myopathies (IIM)

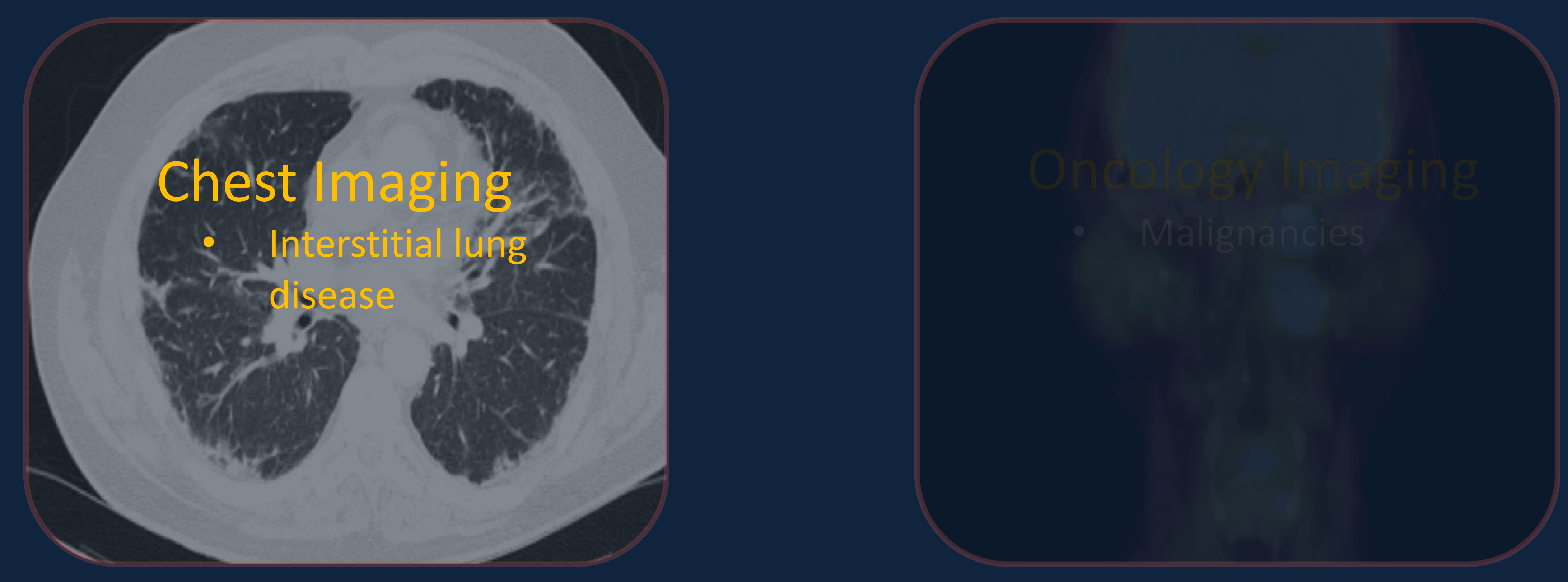




\section{A Note on Interstitial Pneumonia with Autoimmune Features (IPAF)}

- New classification focusing on the relationship between interstitial lung disease (ILD) and connective tissue diseases (CTDs)

- IPAF criteria represent the effort of pulmonologists to classify patients that may remain clinically undefined

- The classification of IPAF combines specific features from three primary domains: clinical, serologic and intrathoracic morphologic features 


\section{Amyotrophic DM (CADM)}

- Strongly associated with refractory, rapidly progressive interstitial lung disease (RP-ILD)

- Clinical and radiographic $\uparrow$ ILD in 3 months

- 2 main ILD patterns:

- RP-ILD/Diffuse alveolar damage (DAD)

- Non-specific interstitial pneumonia (NSIP) +/Organizing pneumonia (OP)

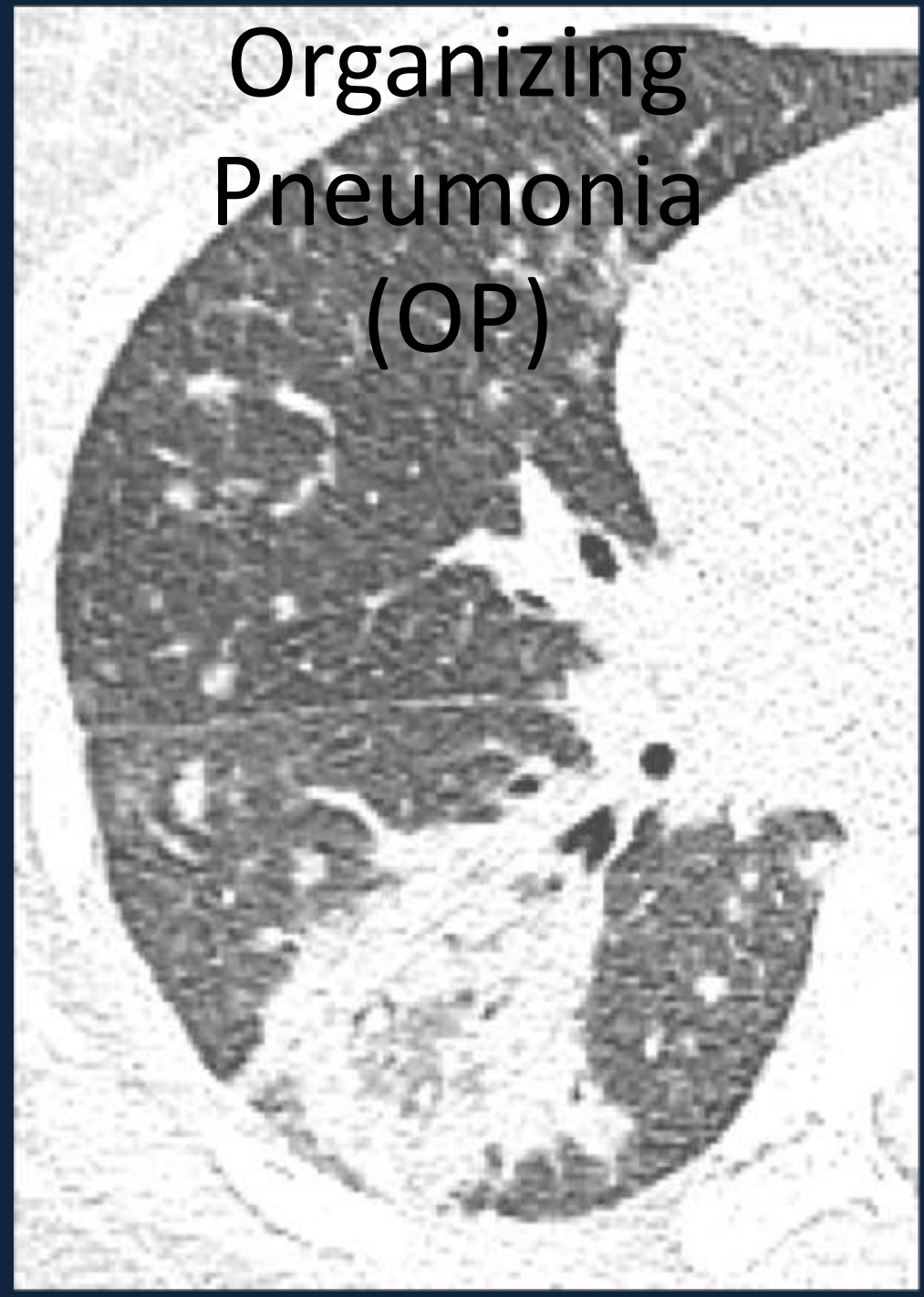

Diffuse Alveolar Damage (DÁD)?

- $\uparrow$ risk of ILD (94\% vs 61\%)

- Strongly associated with RP-ILD

- Anti-MDA5 + : 80\% RPILD, 20\% NSIP/OP

- Anti-MDA5 - : 33\% RPILD, 67\% NSIP/OP

- Single independent predictor of overall mortality

- Anti-MDA5 and concomitant MAAs is associated with $\downarrow$ risk of RP-ILD (33\%)

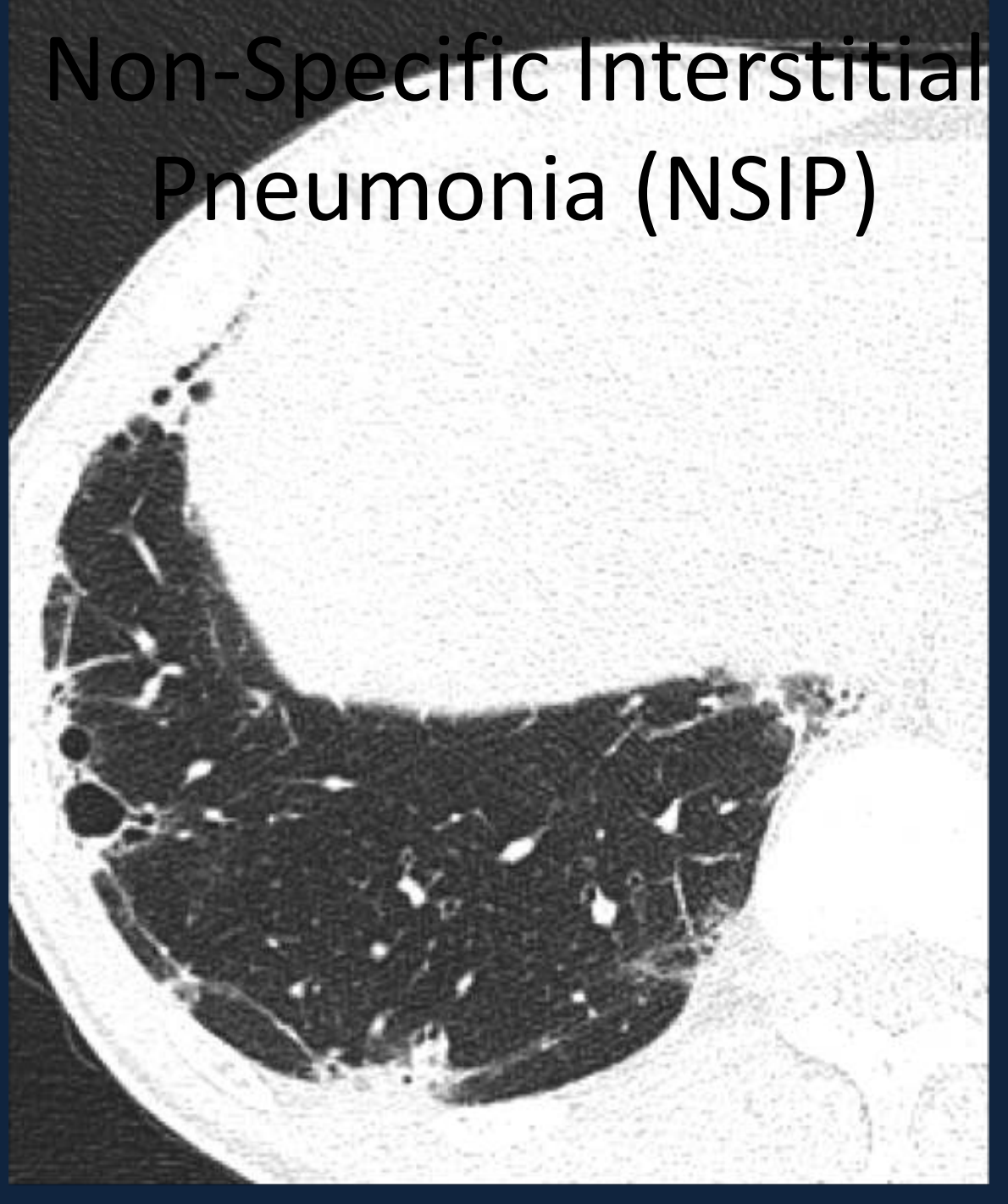

*DAD/RPILD is lower lung predominant (different from AIP/ARDS which is more diffuse) 
Case: $44 \mathrm{M}$, presents with shortness of breath and deteriorating breathing despite corticosteroids
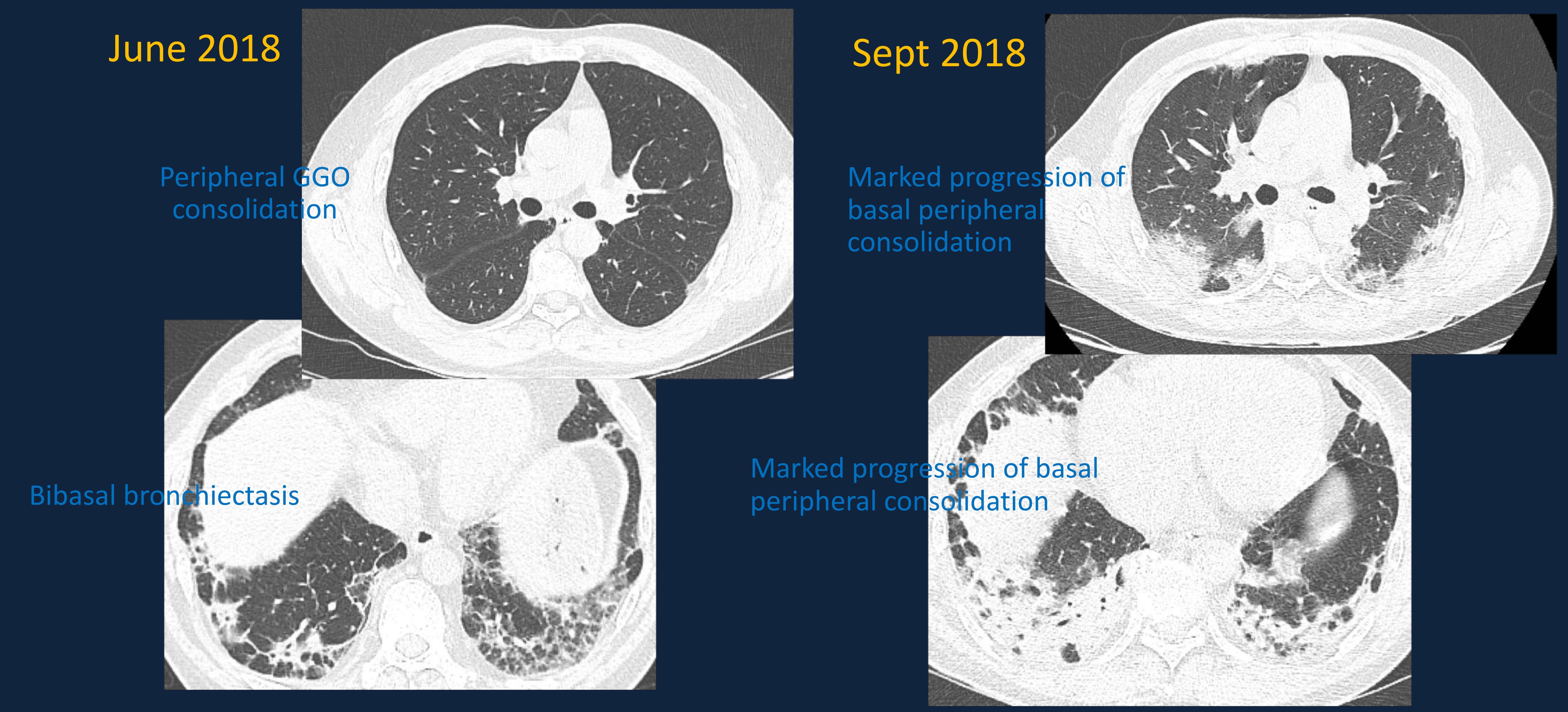

Bronchiectasis in RP-ILD indicates an even worse prognosis similar to AIP

Diagnosis: anti-MDA5+ CADM w/ RP-ILD 
Case: $27 F$ with anti-MDA5+ CADM. Recurrent pneumomediastinum.
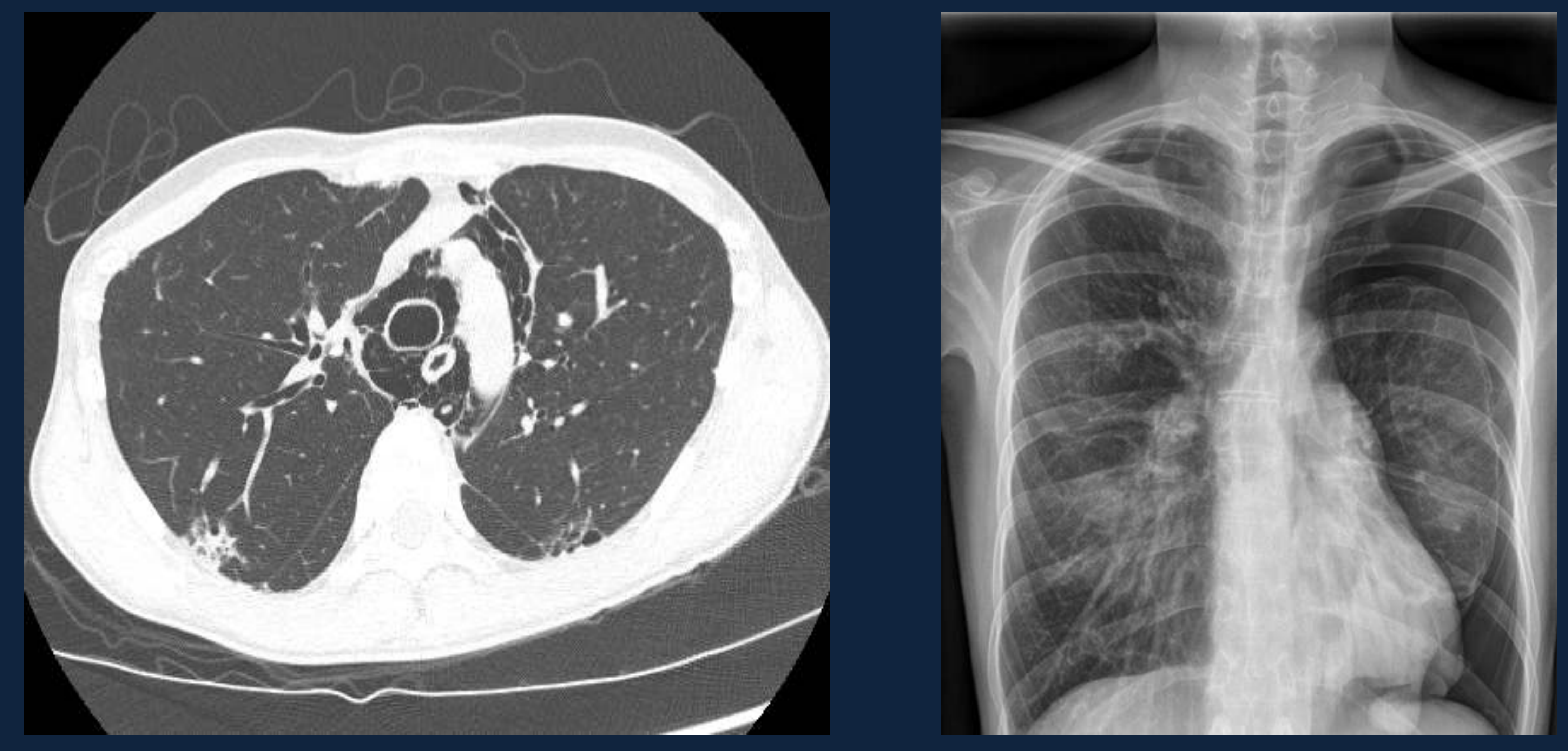

Pneumomediastinum:

- Much more common if anti-MDA5 positive (35\% vs < 2\%)

- Very specific for anti-MDA5+ status (90\%)

- More common in patients that develop RP-ILD (64\%) 


\section{Anti-Synthetase Syndrome (ASS)}

- ILD is a hallmark feature of ASS (70-90\%)

- Patterns are similar amongst all serologies

- NSIP (45-69\%)

- NSIP/OP (24-42\%)

- OP (21\%)

- UIP is very rarely identified on HRCT

- Serologic correlations:

- Anti-Jo1 less frequently has ILD at presentation $(60 \%)$

- Anti-Jo1 and anti-Ro52 cause more severe ILD

- RP-ILD is rare outside anti-MDA5 but has been reported in anti-Jo1 and anti-EJ

- Anti-PL7, PL12, EJ share similar features with more frequent/severe ILD (80-88\%); these serologies are more likely to meet IPAF criteria

- All ASS serologies eventually develop ILD

Patterns of ILD

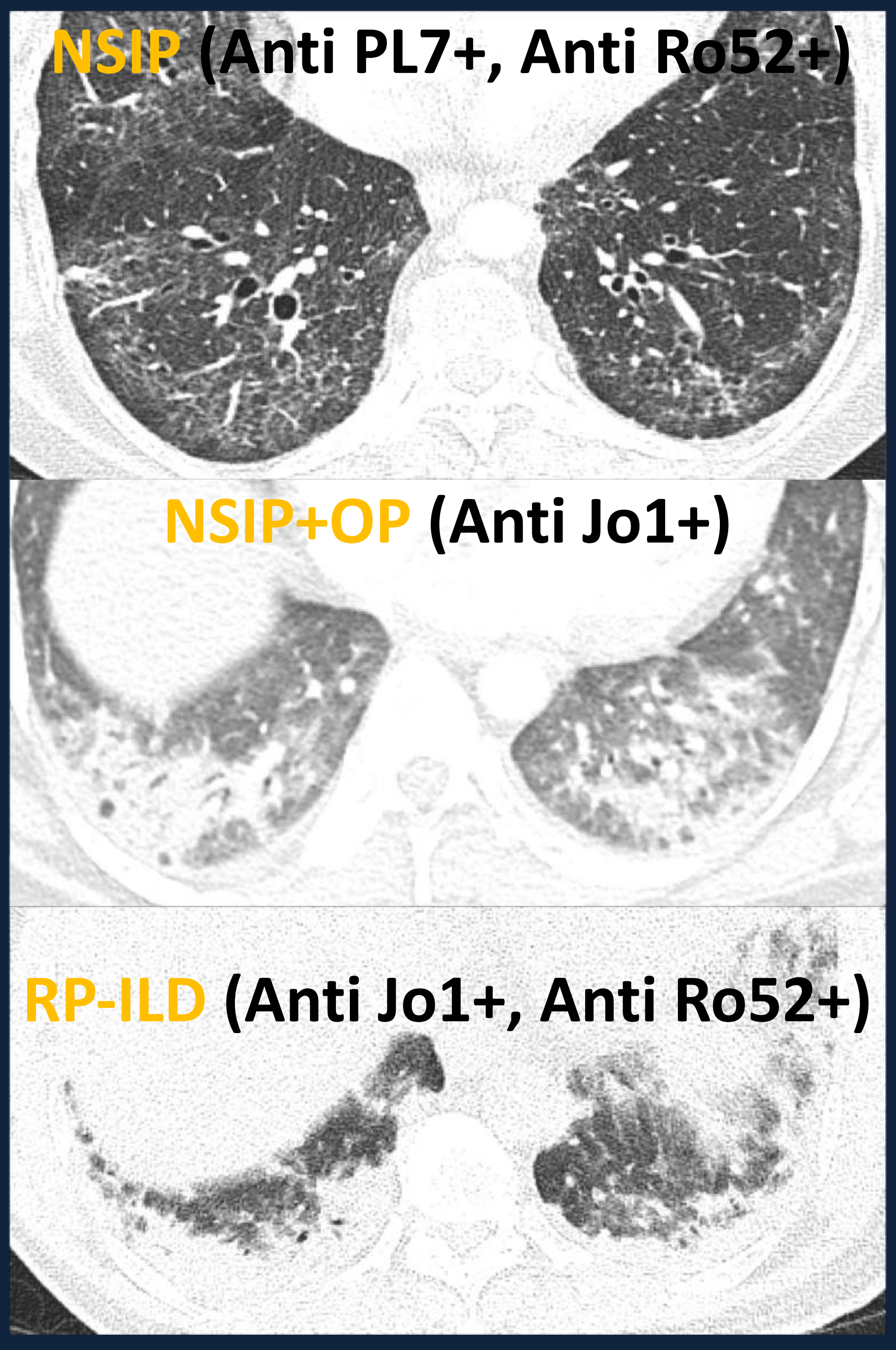




\section{Immune-mediate Necrotizing Myopathy (IMNM)}

Features

- ILD occurs in the minority of patients with IMNM

- Main ILD pattern is NSIP

\section{Serology}

- anti-SRP: $10-20 \%$ risk

- anti-HMGCR: 3\% risk (rare)

\section{Prone CT showing} GGOs with subpleural sparing

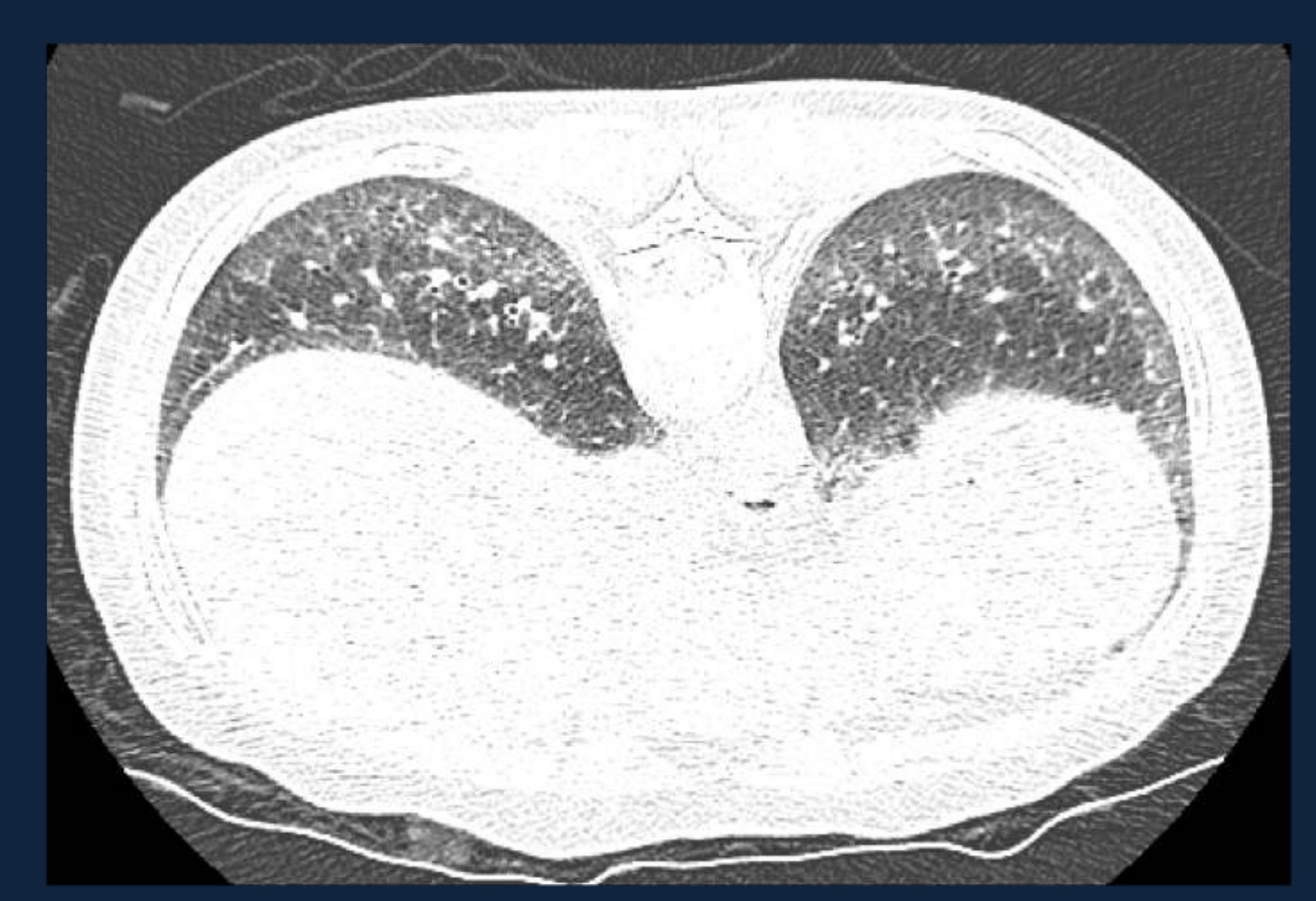

NSIP (anti-SRP)

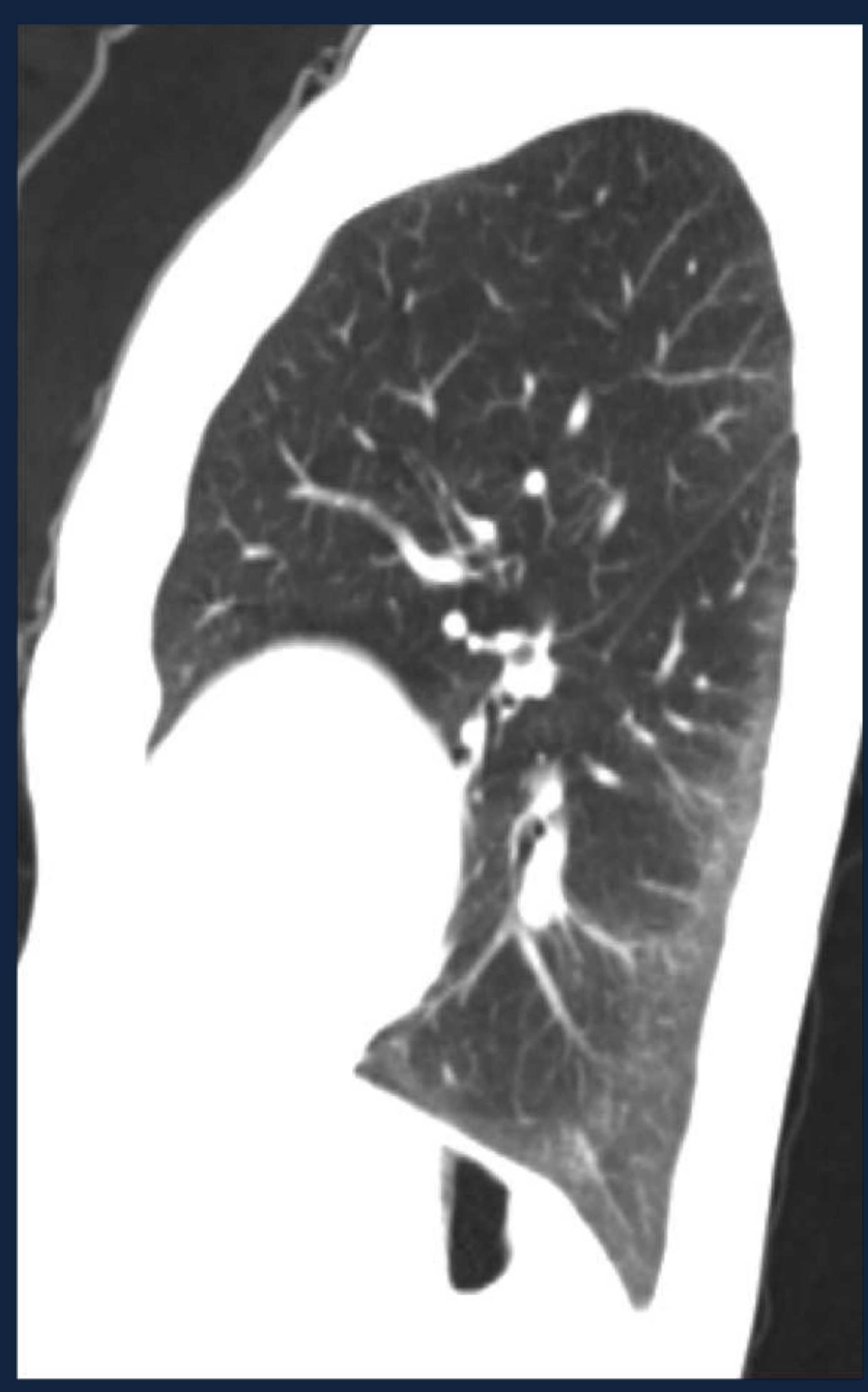

Apical to basal gradient posterior reticulation 
Use of Imaging in Idiopathic Inflammatory Myopathies (IIM)

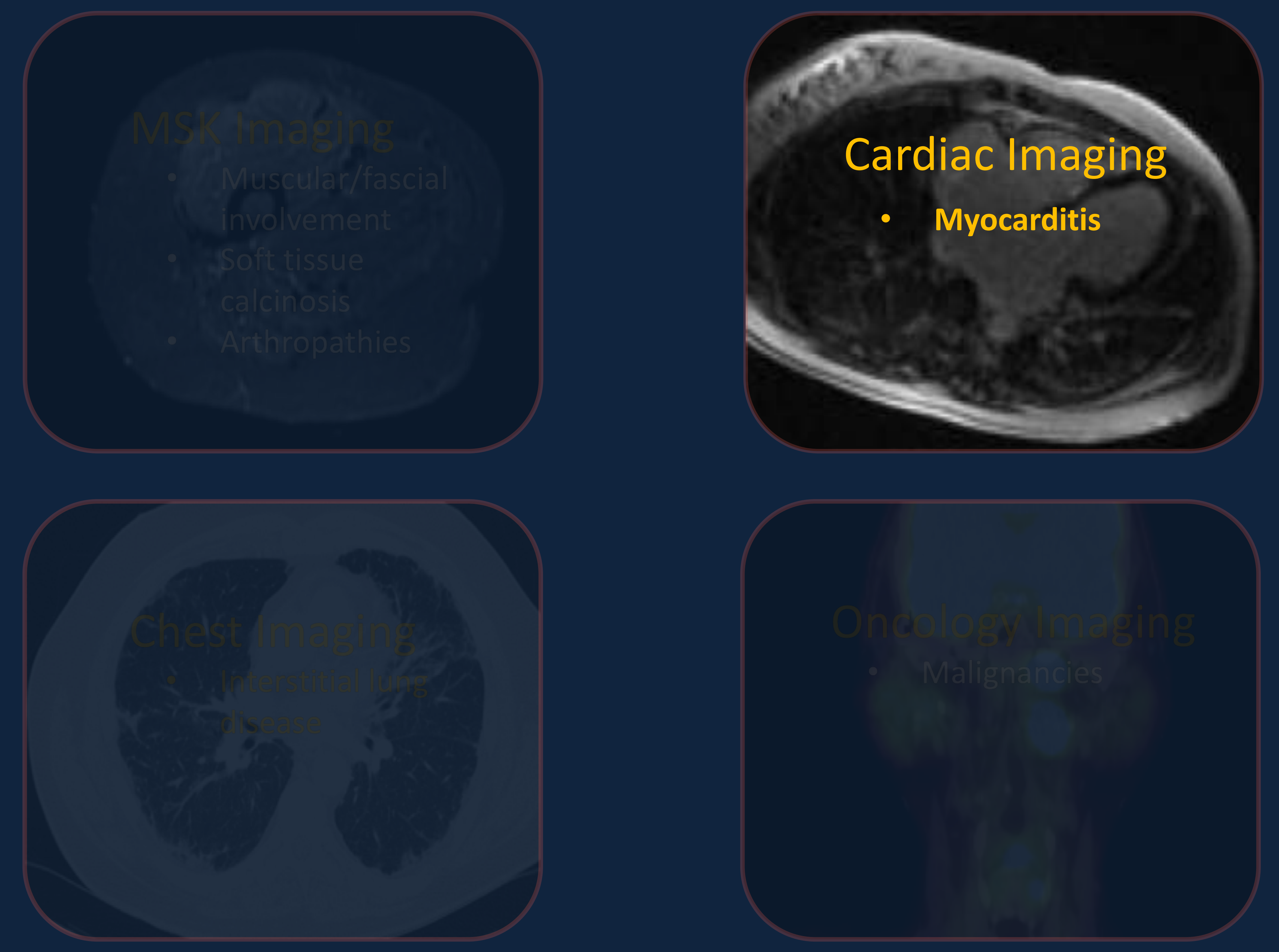




\section{Cardiac Imaging}

Cardiac findings very prevalent but often subclinical/silent

Numerous manifestations:

- Non-specific conduction abnormalities

- Accelerated coronary artery disease

- Pulmonary HTN related to ILD (WHO 3)

- Pericarditis \& pericardial effusion

- Myocarditis

- Systolic and diastolic dysfunction

Prognostically significant

- $10-20 \%$ of all mortality

- $32 \%$ risk of mortality within 8 years

1 Systemic inflammation

Accelerated atherosclerosis

Increased risk of $\mathrm{CAD} / \mathrm{MI}$
2 Interstitial lung disease

Pulmonary hypertension

CTD vasculopathy

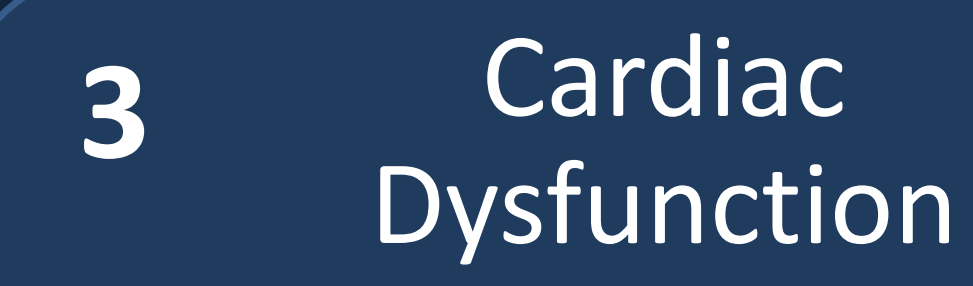

Myo/pericardial inflammation \& fibrosis
Pericarditis

Conduction abnormalities

- Cardiac abnormalities in IIMs is under-studied and likely underreported 


\section{Cardiac Imaging}

Em. Patient symptoms do not correlate presence of late gadolinium enhancement (LGE) on cardiac MR

- Troponin, ECG changes and abnormal TTE are rarely present in patients imaged early after diagnosis, but the majority (40-75\%) meet criteria for myocarditis

\section{Interesting Fact on Troponins}

"Cardiac troponin I (cTnI) should be used instead of cardiac troponin $\mathrm{T}(\mathbf{c T n T})$ in clinical correlation with cardiac imaging findings because it is more specific"

- cTnT is released by diseased skeletal muscle $\rightarrow$ leads to falsepositive troponins in patients with IIMs! 


\section{Cardiac Imaging}

Cardiac MRI Features

- Cardiac MRI (CMR) can be used to diagnose clinically silent myocarditis during acute IIM

- Pattern of myocardial enhancement:

- Patchy distribution

- Spares subendocardium

- Low volume of myocardium involved

-m MUST use expanded CMR criteria for myocarditis

due to abnormal T2 signal and enhancement in

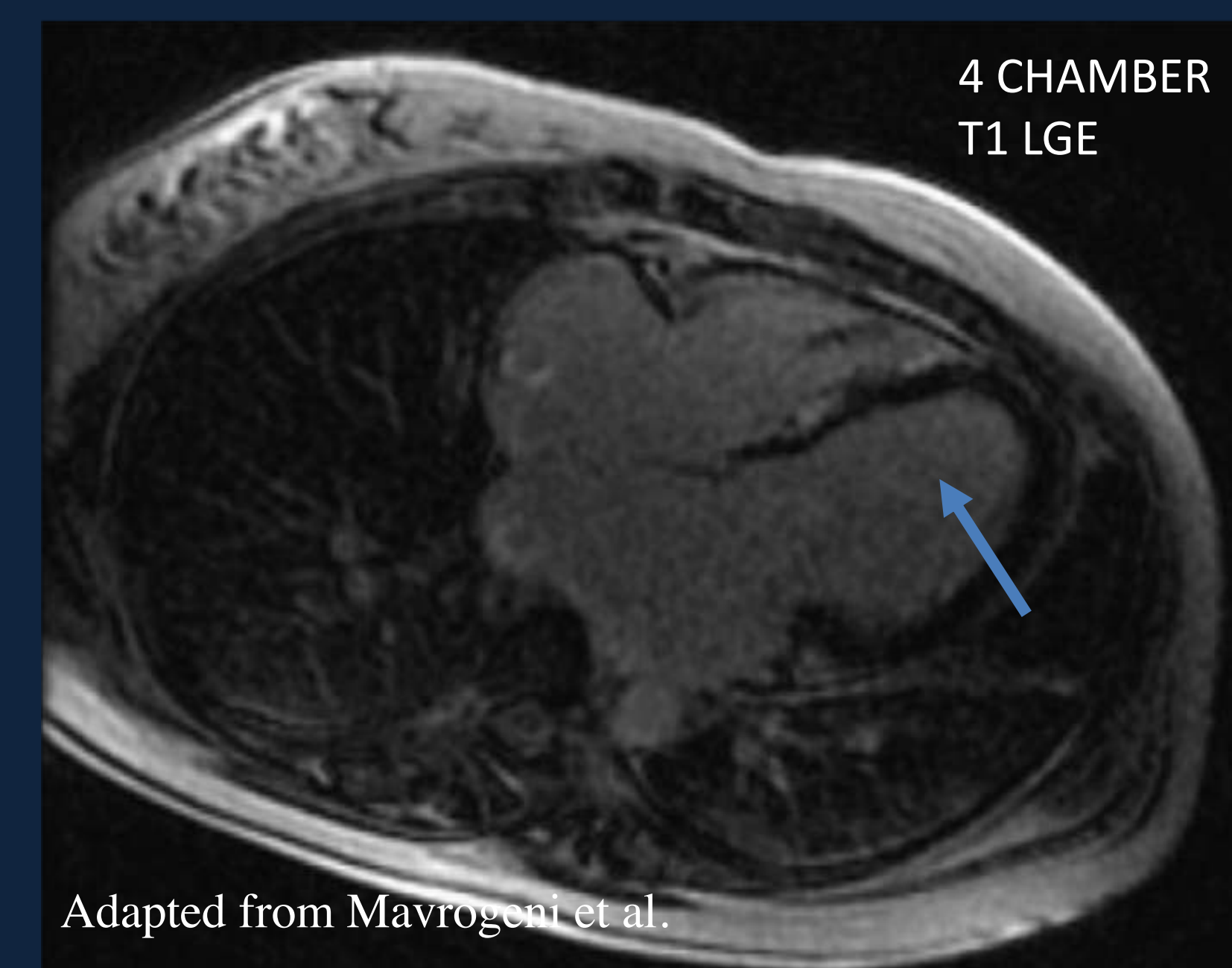

skeletal muscle (detects $20 \%$ more patients)

Intraventricular septum

fibrosis in a patient with acute DM

Criteria (2+ of):

- T2 signal abnormality: ratio or absolute T2 > cut-off (edema)

- Early Gd enhancement: ratio or absolute increase > cut-off (hyperemia)

- Late Gd enhancement: ratio or absolute increase > cut-off (fibrosis) 
Case: 55M with SLE/SSc overlap myositis (OM) and atrial flutter.

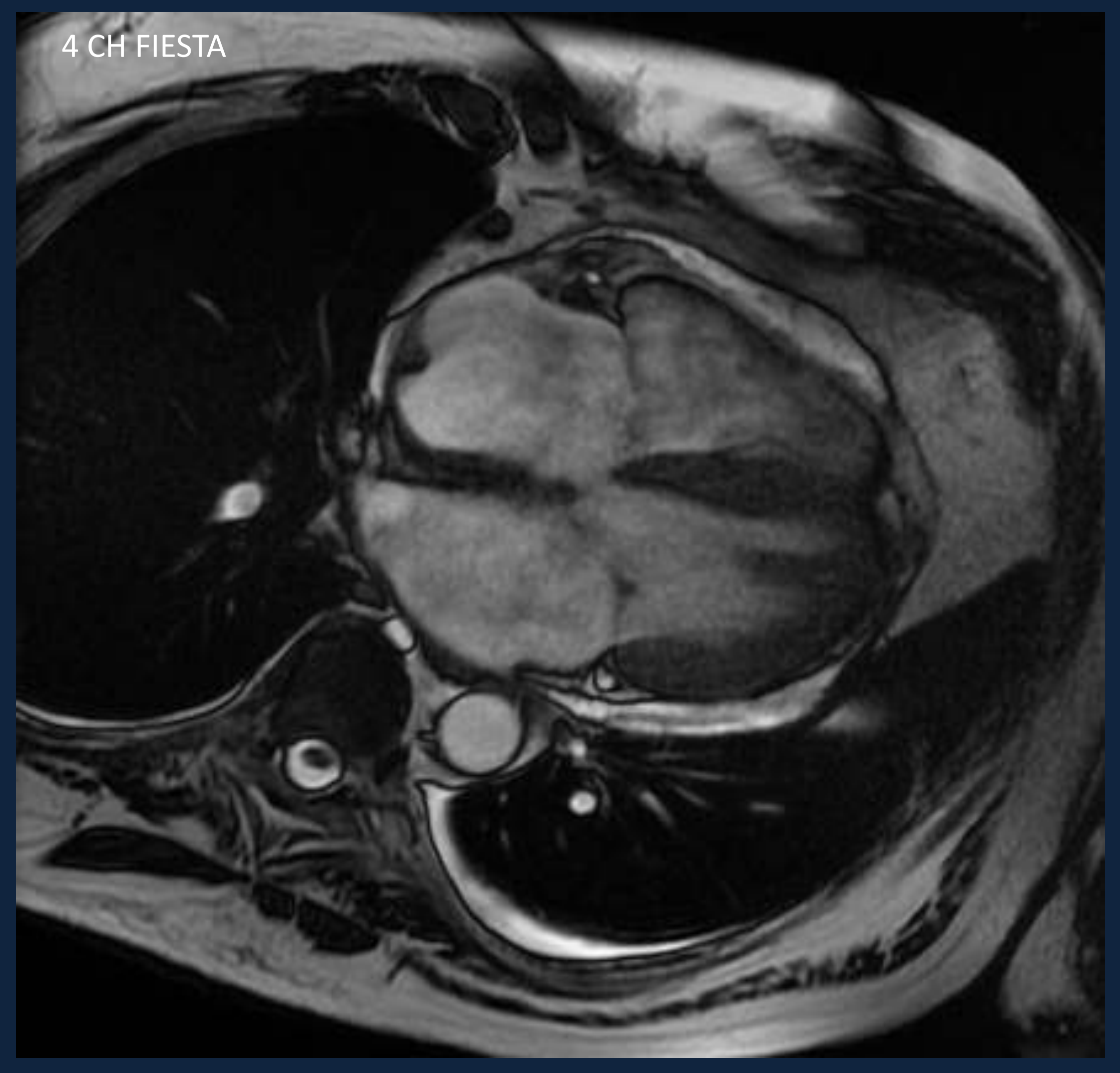

Pericardial effusion

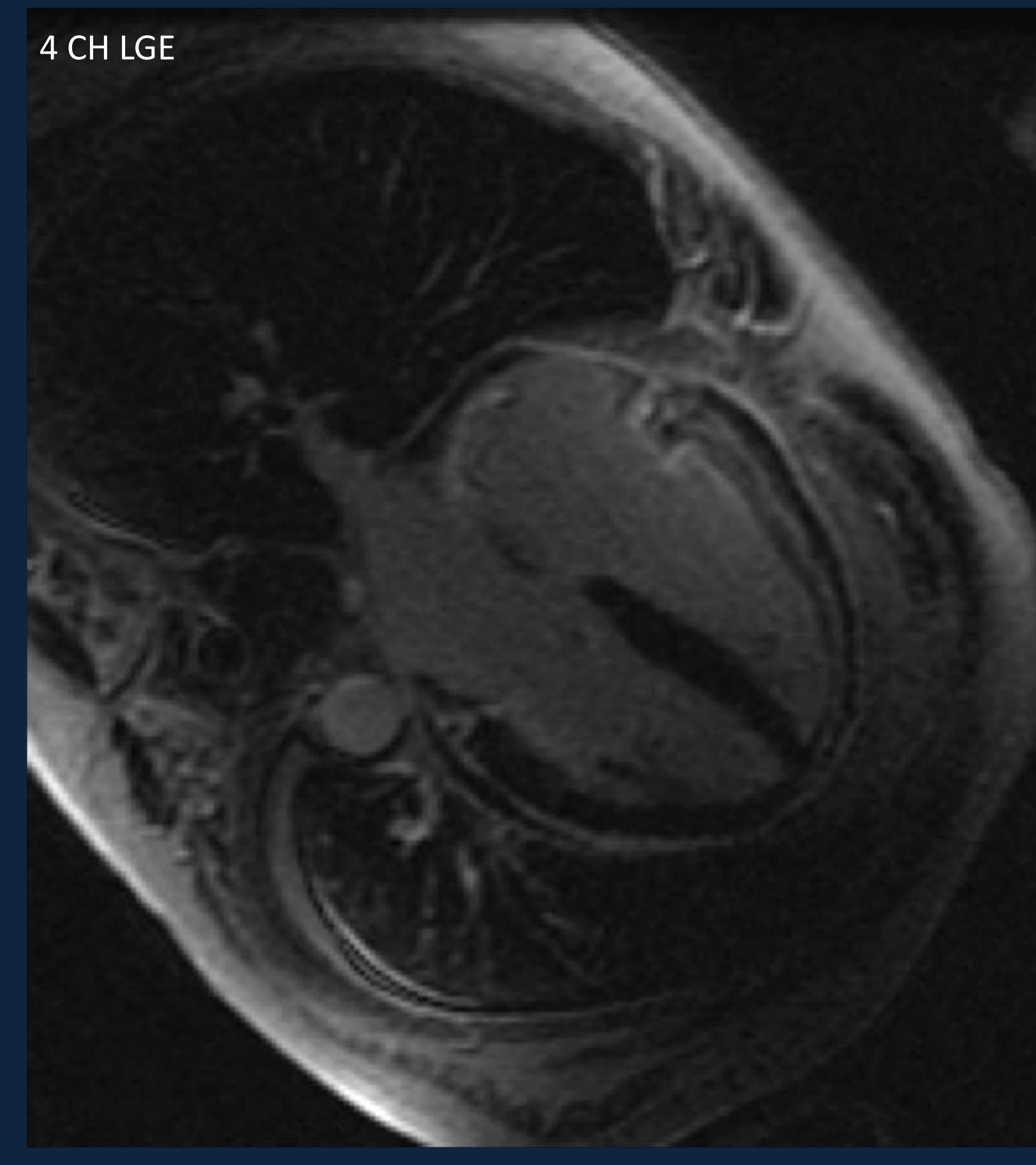

Pericardial enhancement No myocarditis on cardiac MR

Diagnosis: SLE/SSc OM with pericarditis 
Imaging in Idiopathic Inflammatory Myopathies (IIM)

Oncology Imaging

- Malignancies 


\section{IIMs as Paraneoplastic Syndromes}

- IIMs can be part of paraneoplastic syndromes

- $\quad$ risk of malignancy with specific autoantibodies:

- Anti-TIF1g has the strongest association with increased cancer risk (associated with classic DM)

- Sero-negative and anti-HMGCR IMNMs associated with $\uparrow$ cancer risk warranting screening (not anti-SRP IMNM)

- $\quad$ There are no society guidelines regarding specific workup of patients with IIMs for malignancy

- $\quad$ FDG PET/CT should be favored as it is a single study offering convenience with equal effectiveness proven relative to standard organ-specific screening
Ax T2 FS

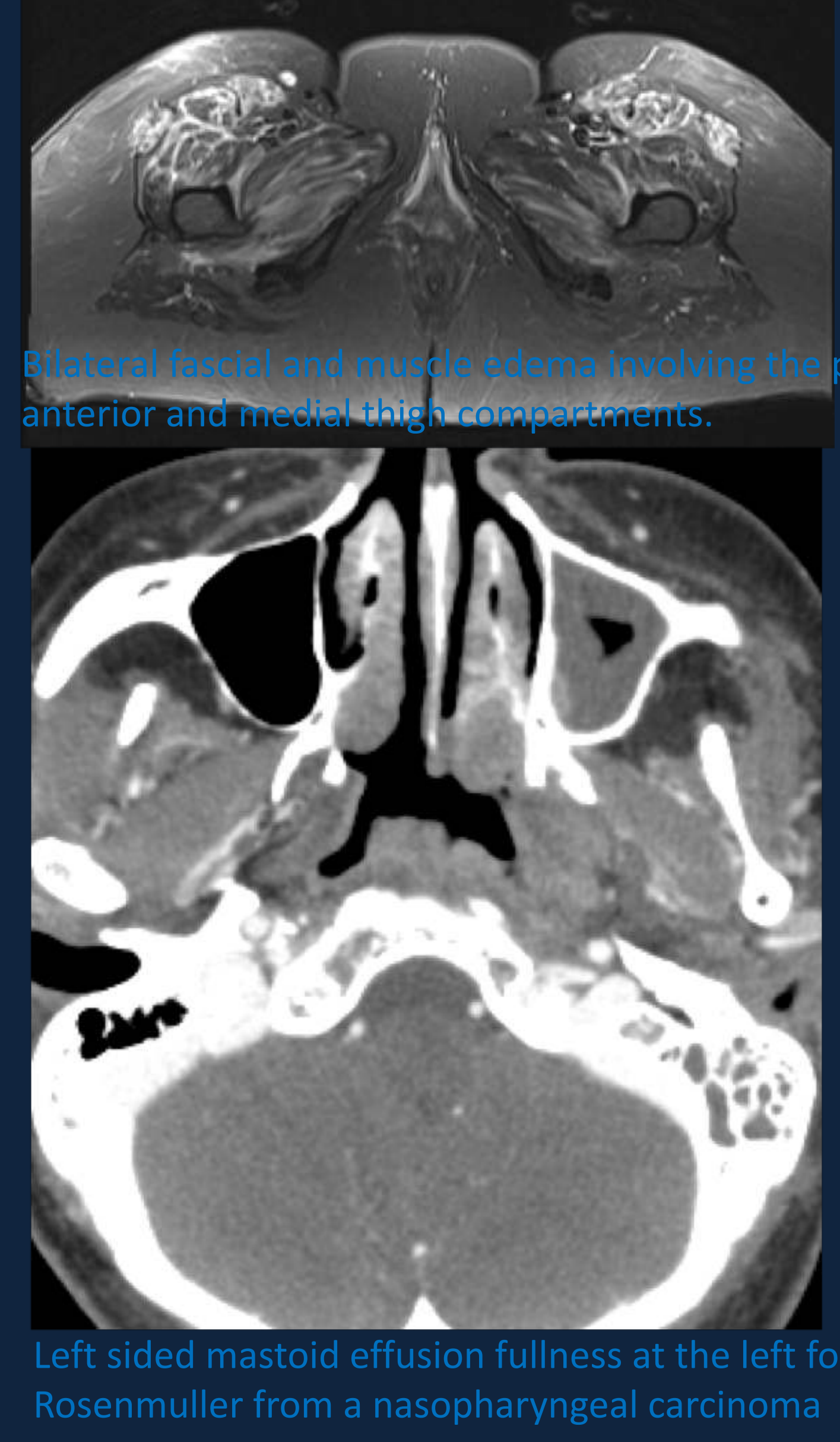

Anti-TIF1g classic DM 
Case: $67 \mathrm{M}$ presents with proximal weakness and working diagnosis of 'polymyositis'. Malignancy screen performed. Preliminary ENA panel negative

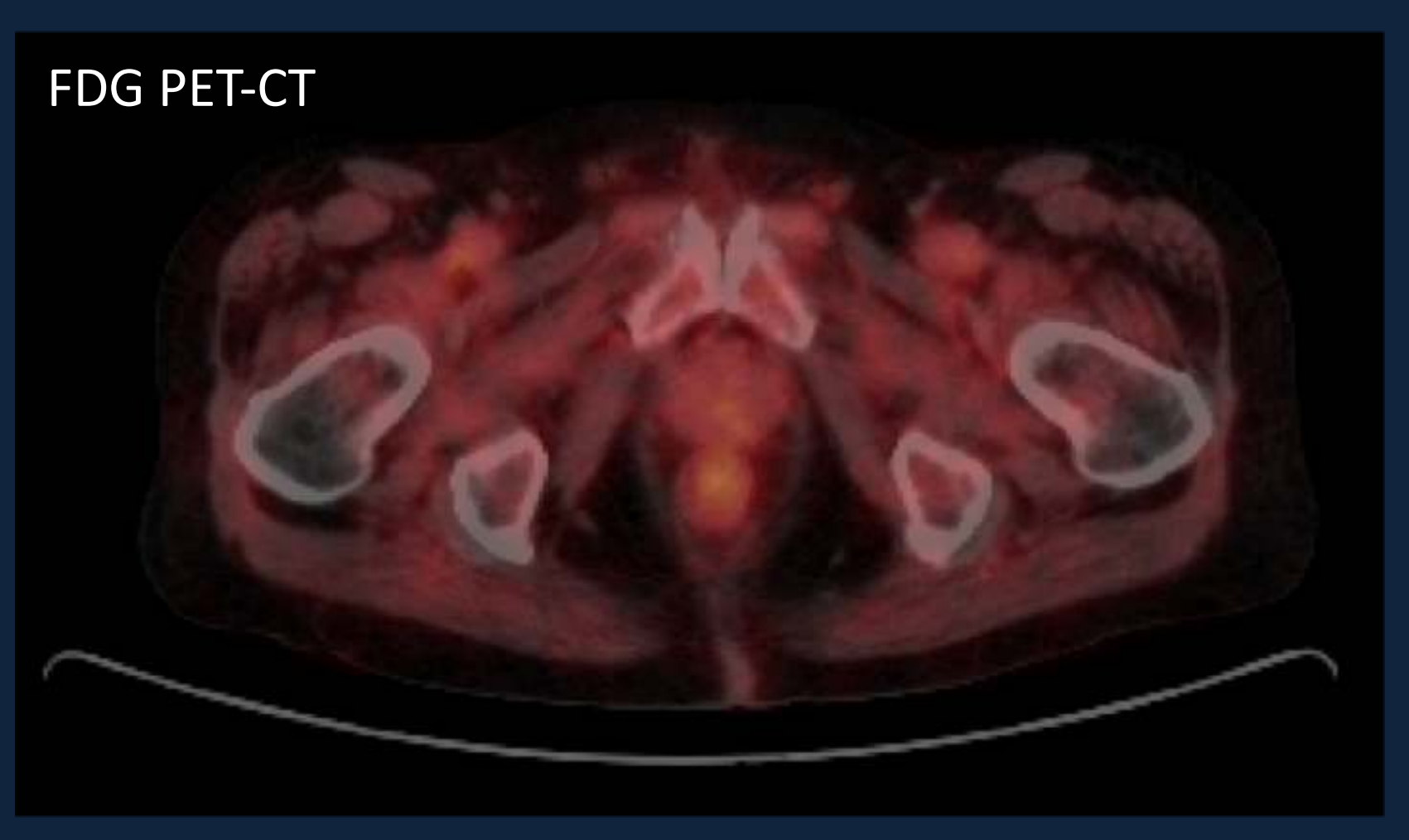

Symmetrical uptake in pelvic muscles on PET-CT

Diagnosis:

Overlap myositis $2^{\text {ndary }}$ to nasopharyngeal carcinoma

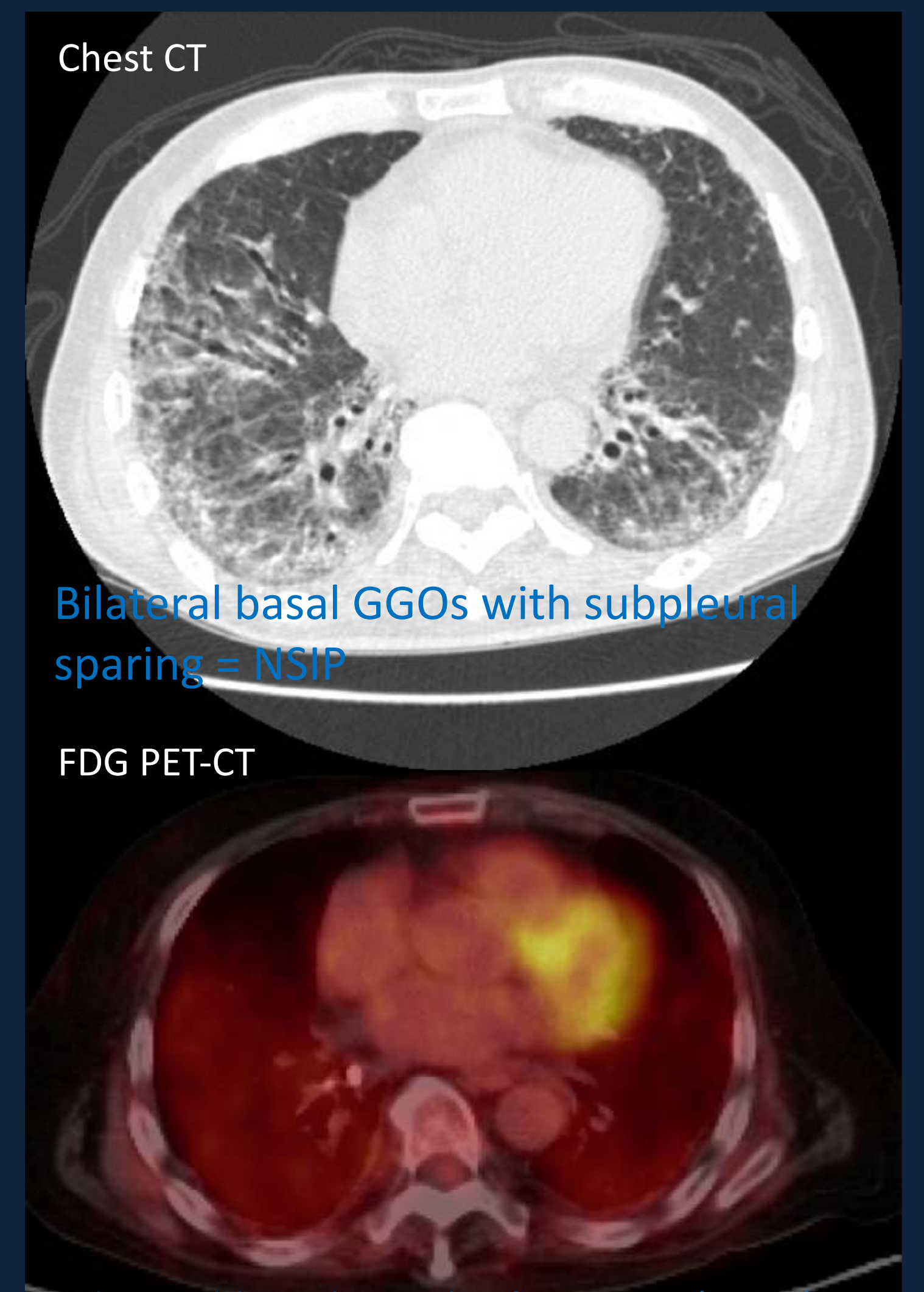

Bitateral basal uptake from ILD (ANSTP) associated with increased disease severity

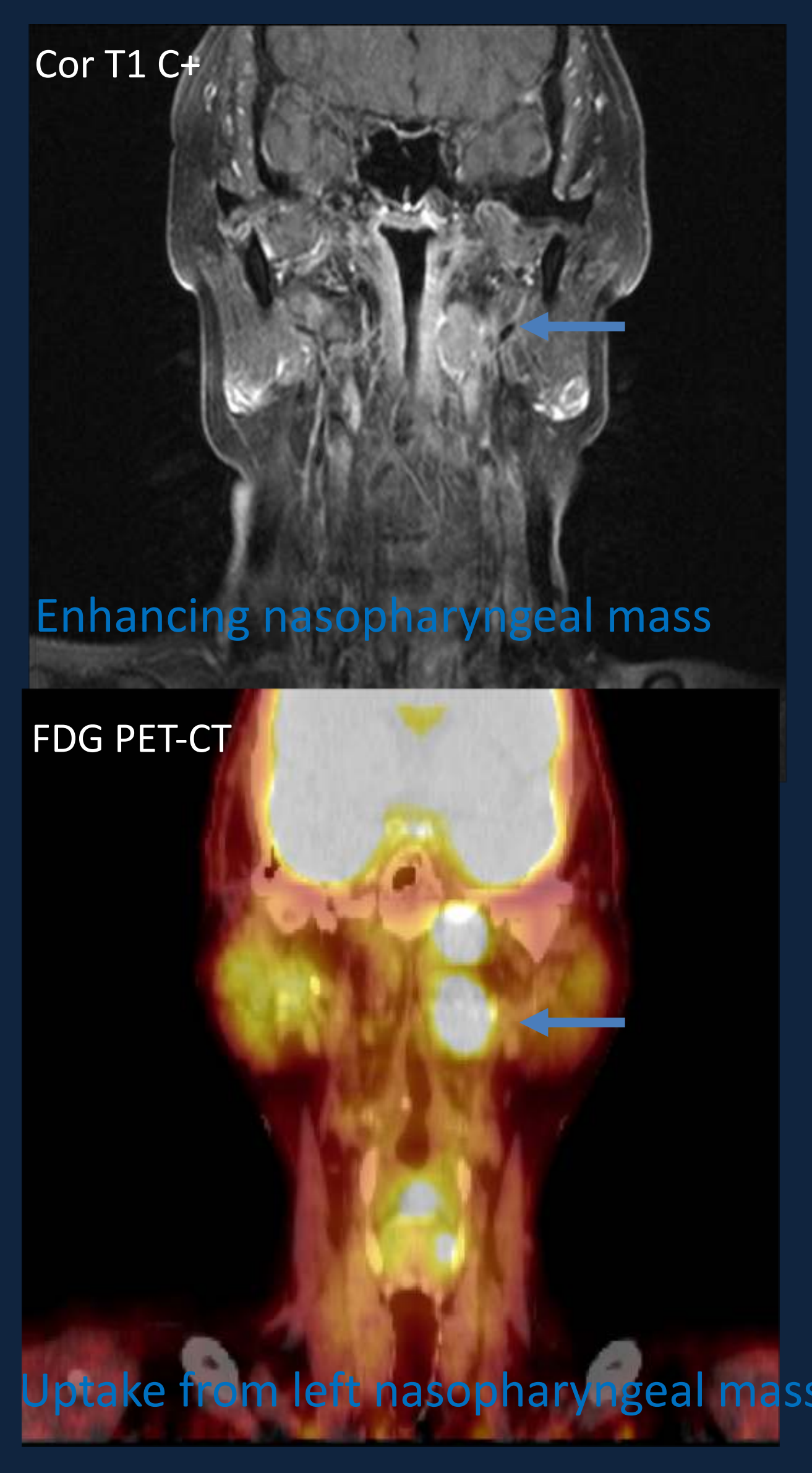


Case: 76F with new diagnosis of NSCLC (2016) develops severe proximal weakness within 6 months
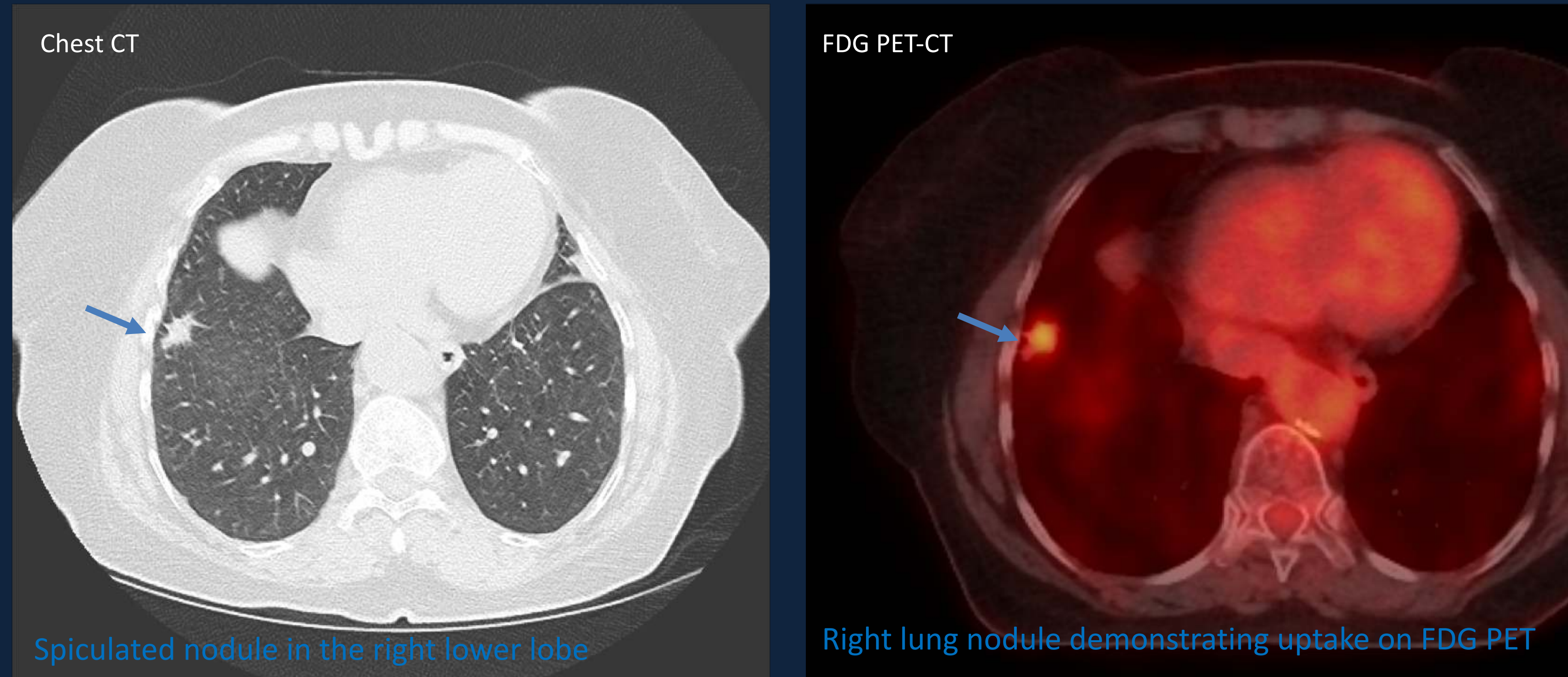

Right lung nodule demonstrating uptake on FDG PET

Diagnosis: Sero-negative IMNM

Cancer diagnosed within 3 years of autoimmune myositis is considered 'cancerassociated' myositis. However, risk of cancer remains elevated for 4-5years in large scale studies 


\section{Summary}

- IIMs should be treated as multi-organ disorders like other CTDs

- Bohan/Peter Classification of IIMs creates 2 groups of patients with heterogenous characteristics, which are parsed into more homogeneous subpopulations with the clinicoserologic approach

- Sensitivity for detection of extra-musculoskeletal abnormalities can be heightened with a working knowledge of serologypathology correlation

- Radiology research in the future should analyze patients groups based on clinicoserologic subtypes 


\section{References}

1. Chino H, Sekine A, Baba T, Iwasawa T, Okudela K, Takemura T, et al. Radiological and Pathological Correlation in AntiMDA5 Antibody-positive Interstitial Lung Disease: Rapidly Progressive Perilobular Opacities and Diffuse Alveolar Damage. Intern Med Tokyo Jpn. 2016;55(16):2241-6.

2. Hozumi H, Fujisawa T, Nakashima R, Johkoh T, Sumikawa H, Murakami A, et al. Comprehensive assessment of myositisspecific autoantibodies in polymyositis/dermatomyositis-associated interstitial lung disease. Respir Med. 2016;121(8908438, rme):91-9.

3. Ito M, Kaise S, Suzuki S, Kazuta Y, Sato Y, Miyata M, et al. Clinico-laboratory characteristics of patients with dermatomyositis accompanied by rapidly progressive interstitial lung disease. Clin Rheumatol. 1999;18(6):462-7.

4. Kang EH, Lee EB, Shin KC, Im CH, Chung DH, Han SK, et al. Interstitial lung disease in patients with polymyositis, dermatomyositis and amyopathic dermatomyositis. Rheumatol Oxf Engl. 2005;44(10):1282-6.

5. Lam WW, Chan H, Chan YL, Fung JW, So NM, Metreweli C. MR imaging in amyopathic dermatomyositis. Acta Radiol Stockh Swed 1987. 1999;40(1):69-72.

6. Yamaguchi K, Yamaguchi A, Kashiwagi C, Sawada Y, Taguchi K, Umetsu K, et al. Differential clinical features of patients with clinically amyopathic dermatomyositis who have circulating anti-MDA5 autoantibodies with or without myositisassociated autoantibodies. Respir Med. 2018;140(8908438, rme):1-5.

7. Ye S, Chen X, Lu X, Wu M, Deng Y, Huang W, et al. Adult clinically amyopathic dermatomyositis with rapid progressive interstitial lung disease: a retrospective cohort study. Clin Rheumatol. 2007 Oct 1;26(10):1647-54.

8. Zhong L, Yu Z, Song H. Association of anti-nuclear matrix protein 2 antibody with complications in patients with idiopathic inflammatory myopathies: A meta-analysis of 20 cohorts. Clin Immunol. 2019 Jan 1;198:11-8.

9. Valenzuela A, Chung L, Casciola-Rosen L, Fiorentino D. Identification of clinical features and autoantibodies associated with calcinosis in dermatomyositis. JAMA Dermatol. 2014 Jul;150(7):724-9.

10. Shahi V, Wetter DA, Howe BM, Ringler MD, Davis MDP. Plain radiography is effective for the detection of calcinosis cutis occurring in association with autoimmune connective tissue disease. Br J Dermatol. 2014;170(5):1073-9.

11. Sanyal S, Atwal SS, Mondal D, Garga UC. Radiographic Patterns of Soft Tissue Calcinosis in Juvenile Dermatomyositis and its Clinical Implications. J Clin Diagn Res JCDR. 2014 Dec;8(12):RD08-RD11.

12. Gutierrez A, Wetter DA. Calcinosis cutis in autoimmune connective tissue diseases. Dermatol Ther. 2012 Apr;25(2):195206.

13. Yang Z, Lin F, Qin B, Liang Y, Zhong R. Polymyositis/dermatomyositis and malignancy risk: a metaanalysis study. J Rheumatol. 2015 Feb;42(2):282-91. 


\section{References}

14. Fredi M, Bartoli F, Cavazzana I, Ceribelli A, Carabellese N, Tincani A, et al. Calcinosis in poly-dermatomyositis: clinical and laboratory predictors and treatment options. Clin Exp Rheumatol. 2017 Apr;35(2):303-8.

15. Blane C, White S, Braunstein E, Bowyer S, Sullivan D. Patterns of calcification in childhood dermatomyositis. Am J Roentgenol. 1984 Feb 1;142(2):397-400.

16. Balin SJ, Wetter DA, Andersen LK, Davis MDP. Calcinosis Cutis Occurring in Association With Autoimmune Connective Tissue Disease: The Mayo Clinic Experience With 78 Patients, 1996-2009. Arch Dermatol. 2012 Apr 1;148(4):455-62.

17. Diederichsen LP, Simonsen JA, Diederichsen AC, Kim WY, Hvidsten S, Hougaard M, et al. Cardiac abnormalities assessed by non-invasive techniques in patients with newly diagnosed idiopathic inflammatory myopathies. Clin Exp Rheumatol. 2015;33(5):706-14.

18. Gupta R, Wayangankar SA, Targoff IN, Hennebry TA. Clinical cardiac involvement in idiopathic inflammatory myopathies: A systematic review. Int J Cardiol. 2011 May 5;148(3):261-70.

19. Haupt HM, Hutchins GM. The heart and cardiac conduction system in polymyositis-dermatomyositis: a clinicopathologic study of 16 autopsied patients. Am J Cardiol. 1982 Nov;50(5):998-1006.

20. Jaffe AS, Vasile VC, Milone M, Saenger AK, Olson KN, Apple FS. Diseased skeletal muscle: a noncardiac source of increased circulating concentrations of cardiac troponin T. J Am Coll Cardiol. 2011 Oct 18;58(17):1819-24.

21. Mavrogeni S, Douskou M, Manoussakis MN. Contrast-enhanced CMR imaging reveals myocardial involvement in idiopathic inflammatory myopathy without cardiac manifestations. JACC Cardiovasc Imaging. 2011 Dec;4(12):1324-5.

22. Mavrogeni S, Bratis K, Karabela G, Stavropoulos E, Sfendouraki E, Kolovou G. Myocarditis during acute inflammatory myopathies: Evaluation using clinical criteria and cardiac magnetic resonance imaging. Int J Cardiol. 2013 Mar 20;164(1):e3-4.

23. Andersson H, Aalokken TM, Gunther A, Mynarek GK, Garen T, Lund MB, et al. Pulmonary Involvement in the Antisynthetase Syndrome: A Comparative Cross-sectional Study. J Rheumatol. 2016;43(6):1107-13.

24. Zenone T, Gibert C, Puget M. Calcinosis and antisynthetase syndrome. Jt Bone Spine Rev Rhum. 2012 Oct;79(5):518. 


\section{References}

25. Andersson H, Kirkhus E, Garen T, Walle-Hansen R, Merckoll E, Molberg O. Comparative analyses of muscle MRI and muscular function in anti-synthetase syndrome patients and matched controls: a cross-sectional study. Arthritis Res Ther. 2017;19(1):17.

26. Debray M-P, Borie R, Revel M-P, Naccache J-M, Khalil A, Toper C, et al. Interstitial lung disease in anti-synthetase syndrome: initial and follow-up CT findings. Eur J Radiol. 2015;84(3):516-23.

27. Hervier B, Uzunhan Y, Hachulla E, Benveniste $\mathrm{O}$, Nunes H, Delaval P, et al. Antisynthetase syndrome positive for antithreonyl-tRNA synthetase (anti-PL7) antibodies. Eur Respir J. 2011 Mar 1;37(3):714-7.

28. Kaneko Y, Kuwana M, Takeuchi T, Oddis CV. Osteolytic change of distal interphalangeal joints and sacroiliac joints in subluxing arthropathy associated with anti-Jo-1 antibody. Jt Bone Spine Rev Rhum. 2013 Oct;80(5):544-5.

29. Lefèvre G, Meyer A, Launay D, Machelart I, DeBandt M, Michaud J, et al. Seronegative polyarthritis revealing antisynthetase syndrome: a multicentre study of 40 patients. Rheumatol Oxf Engl. 2015 May;54(5):927-32.

30. Mejia M, Herrera-Bringas D, Perez-Roman DI, Rivero H, Mateos-Toledo H, Castorena-Garcia P, et al. Interstitial lung disease and myositis-specific and associated autoantibodies: Clinical manifestations, survival and the performance of the new ATS/ERS criteria for interstitial pneumonia with autoimmune features (IPAF). Respir Med. 2017;123(8908438, rme):79-86.

31. Meyer $\mathrm{O}$, Charlanne $\mathrm{H}$, Cherin $\mathrm{P}$, Allanore $\mathrm{Y}$, Coquerelle $\mathrm{P}$, Grardel B, et al. Subluxing arthropathy: an unusual manifestation of the antisynthetase syndrome. Ann Rheum Dis. 2009 Jan 1;68(1):152-3.

32. Noguchi E, Uruha A, Suzuki S, Hamanaka K, Ohnuki Y, Tsugawa J, et al. Skeletal Muscle Involvement in Antisynthetase Syndrome. JAMA Neurol. 2017 Aug 1;74(8):992-9.

33. Oddis CV, Medsger TA, Cooperstein LA. A subluxing arthropathy associated with the anti-Jo-1 antibody in polymyositis/dermatomyositis. Arthritis Rheum. 1990 Nov;33(11):1640-5.

34. Waseda Y, Johkoh T, Egashira R, Sumikawa H, Saeki K, Watanabe S, et al. Antisynthetase syndrome: Pulmonary computed tomography findings of adult patients with antibodies to aminoacyl-tRNA synthetases. Eur J Radiol. 2016;85(8):1421-6. 


\section{References}

35. Schanz S, Henes J, Ulmer A, Kötter I, Fierlbeck G, Claussen CD, et al. Magnetic resonance imaging findings in patients with systemic scleroderma and musculoskeletal symptoms. Eur Radiol. 2013 Jan 1;23(1):212-21.

36. De Lorenzo R, Pinal-Fernandez I, Huang W, Albayda J, Tiniakou E, Johnson C, et al. Muscular and extramuscular clinical features of patients with anti-PM/Scl autoantibodies. Neurology. 2018 Jun 5;90(23):e2068-76.

37. Cavazzana I, Fredi M, Taraborelli M, Quinzanini M, Tincani A, Franceschini F. A subset of systemic sclerosis but not of systemic lupus erythematosus is defined by isolated anti-Ku autoantibodies. Clin Exp Rheumatol. 2013 Apr;31(2 Suppl 76):118-21.

38. Pinal-Fernandez I, Casal-Dominguez M, Carrino JA, Lahouti AH, Basharat P, Albayda J, et al. Thigh muscle MRI in immune-mediated necrotising myopathy: extensive oedema, early muscle damage and role of anti-SRP autoantibodies as a marker of severity. Ann Rheum Dis. 2017;76(4):681-7.

39. Sehgal R, Medina-Flores R, Yachoui R, Kenney CV. Immune Mediated Necrotizing Myopathy: a Cause of Isolated Watanabe Y, Uruha A, Suzuki S, Nakahara J, Hamanaka K, Takayama K, et al. Clinical features and prognosis in anti-SRP and anti-HMGCR necrotising myopathy. J Neurol Neurosurg Psychiatry. 2016;87(10):1038-44.

40. Allenbach Y, Keraen J, Bouvier A, Jooste V, Champtiaux N, Hervier B, et al. High risk of cancer in autoimmune necrotizing myopathies: usefulness of myositis specific antibody. Brain. 2016 Aug 1;139(8):2131-5.

41. Adler RS, Garofalo G. Ultrasound in the evaluation of the inflammatory myopathies. Curr Rheumatol Rep. 2009;11(4):302-8.

42. Bhansing KJ, Rosmalen MHV, Engelen BGV, Vonk MC, Riel PLV, Pillen S. Increased fascial thickness of the deltoid muscle in dermatomyositis and polymyositis: An ultrasound study. Muscle Nerve. 2015;52(4):534-9.

43. Reimers CD, Fleckenstein JL, Witt TN, Müller-Felber W, Pongratz DE. Muscular ultrasound in idiopathic inflammatory myopathies of adults. J Neurol Sci. 1993 May 1;116(1):82-92.

44. Kristensen SB, Hess S, Petersen H, Høilund-Carlsen PF. Clinical value of FDG-PET/CT in suspected paraneoplastic syndromes: a retrospective analysis of 137 patients. Eur J Nucl Med Mol Imaging. 2015 Dec;42(13):2056-63.

45. Selva-O'Callaghan A, Grau JM, Gámez-Cenzano C, Vidaller-Palacín A, Martínez-Gómez X, Trallero-Araguás E, et al. Conventional cancer screening versus PET/CT in dermatomyositis/polymyositis. Am J Med. 2010 Jun;123(6):558-62. 